\title{
Superconductivity in Fullerides
}

\author{
O. Gunnarsson \\ Max-Planck-Institut für Festkörperforschung, D-70506 Stuttgart, Germany
}

Experimental studies of superconductivity properties of fullerides are briefly reviewed. Theoretical calculations of the electron-phonon coupling, in particular for the intramolecular phonons, are discussed extensively. The calculations are compared with coupling constants deduced from a number of different experimental techniques. It is discussed why the $\mathrm{A}_{3} \mathrm{C}_{60}$ are not Mott-Hubbard insulators, in spite of the large Coulomb interaction. Estimates of the Coulomb pseudopotential $\mu^{*}$, describing the effect of the Coulomb repulsion on the superconductivity, as well as possible electronic mechanisms for the superconductivity are reviewed. The calculation of various properties within the Migdal-Eliashberg theory and attempts to go beyond this theory are described.

\section{CONTENTS}

I. Introduction

II. Experimental results

A. Transition temperature

B. Superconductivity gap

C. Isotope effect

D. Other properties

III. Phonons and electron-phonon coupling

A. Intramolecular modes

1. Calculations of phonon frequencies

2. Calculations of electron-phonon coupling

3. Raman and neutron scattering

4. Photoemission

5. Resistivity

6. Comparison of electron-phonon coupling estimates

B. Alkali phonons

C. Librations

D. Intermolecular modes

IV. Coulomb interaction and metallicity

A. Coulomb $U_{0}$ for a free molecule

B. Coulomb $U$ for the solid

C. Mott-Hubbard transition in systems with a large degeneracy

V. Coulomb pseudopotential

A. Retardation effects. Summing ladder diagrams

B. Beyond ladder diagrams

C. Electronic mechanisms

VI. Beyond the Migdal-Eliashberg theory
A. Migdal's theorem
B. Jahn-Teller effects beyond Migdal-Eliashberg treatment

VII. Calculated properties
A. Transition temperature
1. Lattice parameter dependence
2. Doping dependence
B. Isotope effect
C. Reduced gap
D. Other properties

VIII. Concluding remarks

\section{INTRODUCTION}

The fullerenes $\left(\mathrm{C}_{60}, \mathrm{C}_{70}, \ldots\right)$ have attracted much interest since their discovery by Kroto et al. (1985), not least because of their appealing, symmetric shape (see Fig. 1). This interest increased dramatically when Krätschmer et al. (1990) discovered how to produce $\mathrm{C}_{60}$ in large enough quantities to be able to make solids of a size which allowed traditional solid state experiments. Very soon Haddon et al. (1991) found that intercalation of alkali metal atoms in solid $\mathrm{C}_{60}$ leads to metallic behavior. Shortly afterwards it was found that some of these alkali-doped $\mathrm{C}_{60}$ compounds are superconducting with a transition temperature $T_{c}$ which is only surpassed by the cuprates (Hebard et al., 1991; Rosseinsky et al., 1991; Holczer et al., 1991a; Tanigaki et al., 1991; Fleming et al., 1991). Thus $T_{c}$ is $33 \mathrm{~K}$ for $\mathrm{RbCs}_{2} \mathrm{C}_{60}$ (Tanigaki et $a l, 1991)$ and for $\mathrm{Cs}_{3} \mathrm{C}_{60}$ under pressure $T_{c}=40 \mathrm{~K}$ has been reported (Palstra et al, 1995). The great interest in the superconductivity of the alkali-doped $\mathrm{C}_{60}$ compounds is in particular due to these systems being a completely new class of superconductors, the large value of $T_{c}$ and the question whether or not such a large value of $T_{c}$ can be caused by the coupling to phonons alone. There has therefore been a great effort over the last five years to characterize and understand both the normal state and superconducting properties of fullerides. The present review deals only with $\mathrm{C}_{60}$ compounds, since no other superconducting fullerides are known at present.

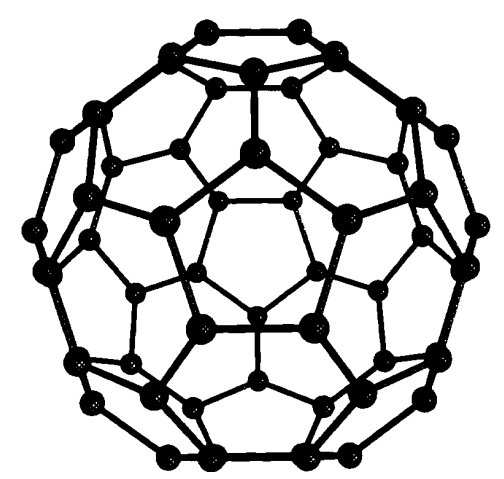

FIG. 1. The $\mathrm{C}_{60}$ molecule. 


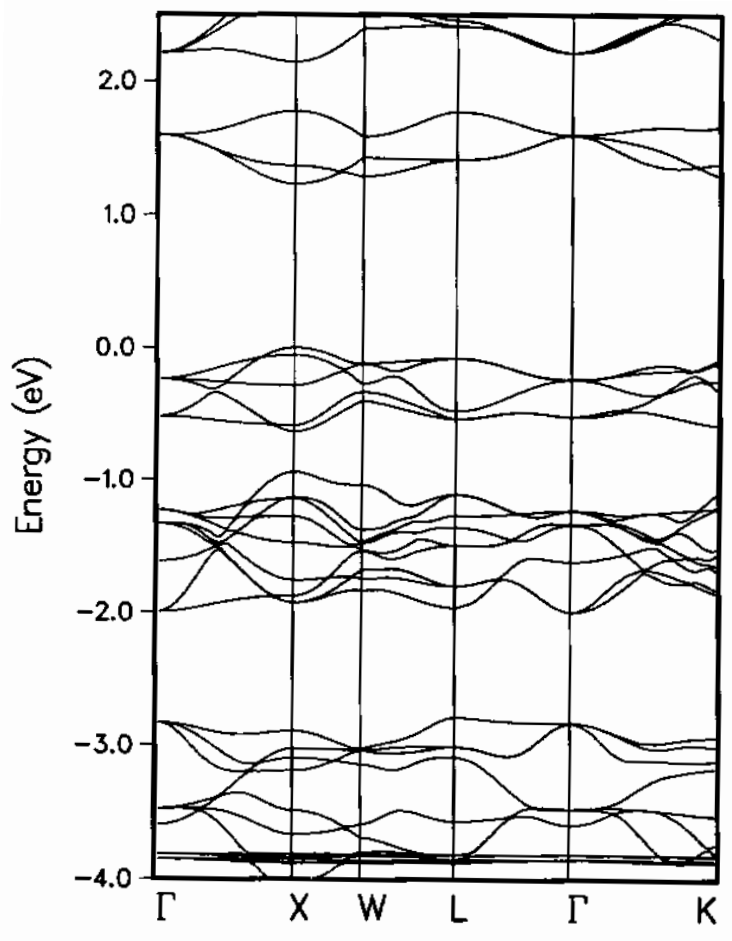

FIG. 2. Some of the subbands around the Fermi energy for solid " $\mathrm{C}_{60}$ in the Fm $\overline{3}$ structure. The bands at about -0.5 $\mathrm{eV}$ are the $h_{u}$ bands, which are occupied in solid $\mathrm{C}_{60}$, and the bands at about $1.5 \mathrm{eV}$ are the $t_{1 u}$ bands, which become populated in $\mathrm{A}_{n} \mathrm{C}_{60}$. The bands around $-1.5 \mathrm{eV}$ results from the overlapping $h_{g}$ and $g_{g}$ bands. (From Erwin (1993).)

$\mathrm{C}_{60}$ is the most symmetric molecule in the sense that its point group (icosahedral) with 120 symmetry operations is the largest point group of the known molecules. It has the shape of a soccer ball. The 60 carbon atoms are all equivalent and form 12 pentagons and 20 hexagons.

The $\mathrm{C}_{60}$ molecules condense to a solid of weakly bound molecules. While the shortest separation between two atoms on the same molecule is about $1.4 \AA$, the shortest separation between two atoms on different molecules is about $3.1 \AA$. The fullerites are therefore molecular solids, where many of the molecular properties essentially survive in the solid. The discrete levels of a free $\mathrm{C}_{60}$ molecule are only weakly broadened in the solid, leading to a set of essentially nonoverlapping bands with a width of about $1 / 2 \mathrm{eV}$ as is illustrated in Fig. 2. The system therefore has two very different energy scales, the intramolecular $\left(E_{I} \sim 30 \mathrm{eV}\right)$ and the intermolecular $(W \sim 1 / 2 \mathrm{eV})$ energy scales. For an undoped $\mathrm{C}_{60}$ solid, the $h_{u}$ band is full and the $t_{1 u}$ band is empty, and this system is therefore a band insulator. When solid $\mathrm{C}_{60}$ is doped by alkali atoms, the alkali atoms donate about one electron each to the $t_{1 u}$ band (Erwin and Pederson, 1991; Saito and Oshiyama, 1991b; Martins and Troullier, 1992; Satpathy et al., 1992). Since the $t_{1 u}$ band can take six electrons, it is half-full for $\mathrm{A}_{3} \mathrm{C}_{60}(\mathrm{~A}=\mathrm{K}, \mathrm{Rb})$, which is a metal (Haddon et al., 1991). $\mathrm{A}_{4} \mathrm{C}_{60}$ is, however, an insulator (Murphy et al., 1992; Kiefl et al., 1992; Benning et al., 1993) although the $t_{1 u}$ band is only partly filled and should be a metal according to band theory (Erwin, 1993; Erwin and Bruder, 1994). $\mathrm{A}_{6} \mathrm{C}_{60}$ is a band insulator. Recently, $\mathrm{AC}_{60}$ has attracted much interest because of its rich and interesting phase diagram and because of its possibly quasi-one dimensional character (Stephens et al., 1994; Chauvet et al., 1994, Erwin et al., 1995). TDAE- $_{60}$ has a ferromagnetic-like transition at $T=16$ $\mathrm{K}$ (Allemand et al., 1991). If the fullerides are considered as organic compounds, they have the highest superconducting and (ferro)magnetic transition temperatures for organic compounds.

In $\mathrm{A}_{3} \mathrm{C}_{60}(\mathrm{~A}=\mathrm{K}, \mathrm{Rb})$ the $\mathrm{C}_{60}$ molecules are located on a fcc lattice. At low temperatures, the $\mathrm{C}_{60}$ molecules take one of two likely orientations in an essentially random way (Stephens et al., 1991). This orientational disorder has a substantial effect on some of the electronic properties (Gelfand and Lu, 1992ab; 1993; Mele and Erwin, 1994), but has been neglected in most calculations. A certain short-range correlation of the orientations is expected on theoretical grounds (Gunnarsson et al., 1991; Mazin et al., 1993a, Erwin and Mele, 1994) and has been observed experimentally (Teslic et al., 1995). The alkali atoms are located in the tetrahedral and octahedral holes. In $\mathrm{Rb} \mathrm{NMR}$ of $\mathrm{Rb}_{3} \mathrm{C}_{60}$ one would then expect to observe two lines. At low temperatures, however, three lines are seen (Walstedt et al., 1993; Zimmer et al., 1993), raising some questions about our understanding of the precise structure. 
The effective Coulomb interaction between two electrons on a $\mathrm{C}_{60}$ molecule in a solid is about $1-1 \frac{1}{2} \mathrm{eV}$ (Lof et al., 1992; Brühwiler et al., 1992; Pederson and Quong, 1992; Antropov et al., 1992). The long-ranged Coulomb interaction in the $\mathrm{A}_{3} \mathrm{C}_{60}$ compounds leads to a charge carrier plasmon, due to the oscillations of the $t_{1 u}$ electrons. This plasmon has the energy $\omega_{p l} \sim 1 / 2 \mathrm{eV}$, an intermediate coupling constant $\left(g / \omega_{p l}\right)^{2} \sim 1$ (Antropov et al., 1993b; Knupfer et al., 1993) and unusal dispersion (Gunnarsson et al., 1996) and broadening (Liechtenstein et al., 1996).

In systems where the superconductivity is driven by the electron-phonon interaction, the transition temperature $T_{c}$ can be calculated in the Eliashberg (1960) theory. A simple estimate of $T_{c}$ can be obtained from the McMillan (1968) formula

$$
T_{c}=\frac{\omega_{l n}}{1.2} \exp \left[-\frac{1.04(1+\lambda)}{\lambda-\mu^{*}(1+0.62 \lambda)}\right],
$$

where $\omega_{l n}$ is a typical phonon frequency (logarithmic average), $\lambda$ is the electron-phonon coupling, and $\mu^{*}$ is the Coulomb pseudopotential, which describes the effects of the repulsive Coulomb interaction. To estimate $T_{c}$ we then in particular need to consider $\lambda$ and $\mu^{*}$.

$\mathrm{C}_{60}$ has intramolecular vibrations (phonons) with energies up to $\omega_{p h} \sim 0.2 \mathrm{eV}$. Only phonons with $\mathrm{A}_{g}$ or $\mathrm{H}_{g}$ symmetry couple to the $t_{1 u}$ electrons. It was very early proposed that these phonons drive the superconductivity (Varma et al., 1991; Schluter et al., 1992ab; Mazin et al., 1992). Estimates of the electron-phonon interaction $\lambda \sim \frac{1}{2}-1$ fall in the right range to explain the experimental values of $T_{c}$. It has therefore become widely, but not universally, accepted that the intramolecular $\mathrm{H}_{g}$ phonons drive the superconductivity. Other phonon modes, such as librations, $\mathrm{C}_{60}-\mathrm{C}_{60}$ and alkali- $\mathrm{C}_{60}$ vibrations are believed to play a small role for the superconductivity. It has, however, also been argued that an electronic mechanism may drive the superconductivity (Chakravarty et al., 1991; Baskaran and Tosatti, 1991; Friedberg et al., 1992).

The attractive interaction between two electrons induced by the electron-phonon interaction is small $(\sim$ $1 / 10 \mathrm{eV}$ ) compared with the Coulomb repulsion between two electrons on the same $\mathrm{C}_{60}$ molecule $\left(U \sim 1-1 \frac{1}{2}\right.$ $\mathrm{eV})$. Nevertheless, for conventional superconductors it is argued that the effects of the Coulomb interaction are drastically reduced by retardation effects, due to the very different energy scales for phonons and electrons. The dimensionless Coulomb pseudopotential $\mu^{*}$ is therefore believed to be drastically renormalized for such systems. For doped $\mathrm{C}_{60}$ solids it has been controversial whether the intramolecular $\left(E_{I}\right)$ or intermolecular $(W)$ (Anderson, 1991) energy scale is relevant for the retardation effects. In the latter case one may expect the retardation effects to be small, since $\omega_{p h} \sim W$. It has there- fore been asserted that the phonons alone cannot explain the superconductivity in $\mathrm{A}_{3} \mathrm{C}_{60}$ (Anderson, 1991). Later work has provided support for the intermolecular energy scale being the relevant one, but nevertheless found that the electron-phonon mechanism may be sufficient if the Coulomb interaction is screened as efficiently as predicted by RPA (Gunnarsson and Zwicknagl, 1992).

In the $\mathrm{A}_{3} \mathrm{C}_{60}(\mathrm{~A}=\mathrm{K}, \mathrm{Rb})$ compounds many energy scales are similar $\omega_{p h} \sim \tilde{E}_{J T} \lesssim \omega_{p l} \sim W \lesssim U$, where $\tilde{E}_{J T}$ is the Jahn-Teller energy of $\mathrm{C}_{60}^{-3}$, which may be of the order of several tenths of an eV. Furthermore, certain coupling constants are intermediate $\left.(\lambda \sim 1),\left(g / \omega_{p l}\right)^{2} \sim 1\right)$. This is a situation where we may expect particularly interesting physics, but also a situation which is particularly difficult to treat theoretically. $W<U$ suggests very strong correlation effects (Lof et al., 1992), which have, however, not been considered in most treatment of fullerides. The similar magnitude of $\omega_{p h}$ and $W$ raises doubt about the strength of the retardation effects, as discussed above, and it implies that Migdal's theorem is not valid. Corrections to Migdal's theorem have been considered in a few cases (see, e.g., Pietronero al., 1995, Takada 1993). Jahn-Teller-like effects, beyond what is included in the Migdal theory, may also play a role (Auerbach et al., 1994; Manani et al., 1994). The fact that $\tilde{E}_{J T}$ may not be much smaller than $W$ could also be important for the insulating character of $\mathrm{A}_{4} \mathrm{C}_{60}$ (Fabrizio and Tosatti, 1996). In view of this, it is not surprising that many of the normal state properties are unusual (see, e.g., Sawatzky (1995)) and several interesting, unresolved questions remain.

There have been a number of reviews of the fullerenes. Several articles appeared in J. Chem. Phys. Solid Vol. 53, No. 12 (Fischer and Cox, 1992), in Vol. 30 No. 8 of Carbon (1992), in Vol. 48 of Solid State Physics (1994), e.g., by Pickett (1994) and by Lieber and Zhang (1994), and in a book edited by Billups and Ciufolini (1993). Early reviews were given by Weltner et al. (1989), by Kroto et al. (1991) by Wilson et al. (1992) and by Loktev (1992). A number of conference proceedings have appeared (Hammond and Kuck, 1992; Kumar et al., 1993; Fischer et al., 1993; Prassides et al., 1994, Kuzmany et al., 1993; 1994a; 1995a). The field has further been reviewed by Holczer and Whetten (1992) and by Ramirez (1994) from an experimental and by Gelfand (1994) from a theoretical point of view. A general review with the emphasis on the superconductivity was given by Hebard (1992). Specific topics have recently been review by Golden et al. (1995) (high energy spectroscopies), Kuzmany et al. (1995b) (infrared spectroscopies), Pintschovius (1996) (neutron scattering) Kuzmany et al. (1994b) (Raman scattering) and Buntar and Weber (1996) (magnetic properties). The theory of superconductivity in general has been presented in several text-books, e.g., Schrieffer (1964), Parks (1969) and Tin- 
kham (1975), and in reviews, e.g., Allen and Mitrovic (1982) and Carbotte (1990), and it will not be addressed here. Popular descriptions of the fullerenes have been given by Baggot (1994) and Aldersey-Williams (1995).

TABLE I. Structure, valence and band involved for certain classes of fullerene superconductors. ( $\mathrm{AE}=$ Alkaline Earth, $\mathrm{RE}=$ Rare Earth)

\begin{tabular}{lll}
\hline \hline Class & Structure & Valence \\
\hline $\mathrm{A}_{3} \mathrm{C}_{60}$ & $\mathrm{Fm} \overline{3} \mathrm{~m}$ & $n=3\left(t_{1 u}\right)$ \\
$\mathrm{Na}_{2} \mathrm{~A}_{x} \mathrm{C}_{60}$ & $\mathrm{~Pa} 3$ & $n \leq 3\left(t_{1 u}\right)$ \\
$\mathrm{A}_{3-x} \mathrm{Ba}_{x} \mathrm{C}_{60}$ & $\mathrm{Fm} \overline{3} \mathrm{~m}$ & $n \geq 3\left(t_{1 u}\right)$ \\
$(\mathrm{AE})_{x} \mathrm{C}_{60}$ & $\operatorname{Im} \overline{3}$, sc, orthorhomb & $n>6\left(t_{1 g}\right)$ \\
$(\mathrm{RE})_{x} \mathrm{C}_{60}$ & orthorhomb & \\
\hline \hline
\end{tabular}

\section{EXPERIMENTAL RESULTS}

\section{A. Transition temperature}

The superconducting fullerides may be divided in five classes; 1) $\mathrm{A}_{3} \mathrm{C}_{60}(\mathrm{~A}=\mathrm{K}, \mathrm{Rb}, \mathrm{Cs}$ or some combination of these elements, 2) $\mathrm{Na}_{2} \mathrm{~A}_{x} \mathrm{C}_{60}(x \leq 1)(\mathrm{A}=\mathrm{K}, \mathrm{Rb}$, Cs or a combination of these, 3) $\mathrm{A}_{3-x} \mathrm{Ba}_{x} \mathrm{C}_{60}(\mathrm{~A}=\mathrm{K}, \mathrm{Rb}, \mathrm{Cs})$, 4) $\mathrm{Ca}_{x} \mathrm{C}_{60}(x \sim 5)$ (Kortan et al., 1992a) and $\mathrm{Ba}_{6} \mathrm{C}_{60}$ (Kortan et al., 1992b) or $\mathrm{Ba}_{4} \mathrm{C}_{60}$ (Baenitz et al., 1995) and $\mathrm{Sr}_{6} \mathrm{C}_{60}$ (Kortan et al., 1994) and 5) $\mathrm{Yb}_{2.75} \mathrm{C}_{60}$ and $\mathrm{Sm}_{x} \mathrm{C}_{60}$. A summary is given in Table I.

These classes differ in particular in their structure and the $\mathrm{C}_{60}$ valence. In the first class and in the second class with $x=1$ three electrons are doped into the $t_{1 u}$ band, while in the second class with $x<1$ the doping is less than three electrons. In the third class the filling of the $t_{1 u}$ band is larger than three and in the fourth class the $t_{1 u}$ band is full and the $t_{1 g}$ band is populated (Chen et al., 1992; Wertheim et al., 1992; Erwin and Pederson, 1993; Saito and Oshiyama, 1993; Knupfer et al., 1994). The $t_{1 g}$ states furthermore strongly hybridize with alkaline earth states (Chen et al., 1992; Erwin and Pederson, 1993; Saito and Oshiyama, 1993), in contrast to the very weak hybridization of the $t_{1 u}$ states with the alkali states in $\mathrm{A}_{3} \mathrm{C}_{60}$.

In the first three classes, the $\mathrm{C}_{60}$ molecules are located on a fcc lattice. The first (Stephens et al., 1991) and third (Yildirim, 1996b) class have the space group Fm $\overline{3} \mathrm{~m}$, and the molecules more or less randomly take one of two preferential orientations. In the second class the space group is $\mathrm{Pa} \overline{3}$ (Kniaz et al., 1993; Prassides et al., 1994), possibly with some distortions for $\mathrm{Na}_{2} \mathrm{KC}_{60}$ and $\mathrm{Na}_{2} \mathrm{RbC}_{60}$ (Maniwa et al., 1995). For these systems there are four $\mathrm{C}_{60}$ molecules per unit cell, all having different orientations. In the fourth group $\mathrm{Ca}_{5} \mathrm{C}_{60}$ has a simple cubic structure with the molecules at the fcc lattice positions (Kortan et al., 1992a), $\mathrm{Ba}_{4} \mathrm{C}_{60}$ has an orthorhombic structure (Baenitz et al., 1995), $\mathrm{Ba}_{6} \mathrm{C}_{60}$ (Kortan et al., $1992 \mathrm{~b}$ ) and $\mathrm{Sr}_{6} \mathrm{C}_{60}$ (Kortan et al., 1994) have a bcc structure $(\operatorname{Im} \overline{3})$.

The $T_{c}$ as a function of the lattice parameter $a$ has been determined by a large number of groups for both the first (Tanigaki et al., 1991; Sparn et al., 1991; Fleming et al., 1991; Zhou et al., 1992) and second (Tanigaki et al., 1992; Yildirim et al., 1994; Mizuki et al., 1994, Yildirim et al., 1995) (for $x=1$ ) class. In Fig. 3 these results are summarized. The figure illustrates that in the first class, $T_{c}$ varies smoothly with $a$, relatively independently of the alkali atoms in the compound. Thus if pressure is applied to $\mathrm{Rb}_{3} \mathrm{C}_{60}$ to give the same $a$ as for $\mathrm{K}_{3} \mathrm{C}_{60}$ at normal pressure, the corresponding $T_{c}$ 's are approximately equal (Zhou, 1992). It is therefore the lattice parameter, not the alkali atom, which determines $T_{c}$. This seems to exclude the alkali vibrations as the main driving force for the superconductivity, since the vibration frequency and thereby $T_{c}$ would then be expected to depend on the mass of the alkali atoms. Recent work has, however, questioned these results, finding a different pressure-volume relation for $\mathrm{Rb}_{3} \mathrm{C}_{60}$ than the earlier work and thereby a different $T_{c}-a$ dependence (Diederichs et al., 1996). A certain deviation between $\mathrm{Rb}_{2} \mathrm{CsC}_{60}$ under pressure and $\mathrm{Rb}_{3} \mathrm{C}_{60}$ was also observed by Schirber et al. (1993). This would imply that changing the lattice parameter by physical or chemical pressure does not lead to identical results, although the deviations are not dramatic. A smooth variation with lattice parameter is observed for the second group $(x=1)$ (see, Fig. 3 ). It is, however, interesting that the slopes of the two classes are very different.

$\left(\mathrm{NH}_{3}\right)_{4} \mathrm{Na}_{2} \mathrm{CsC}_{60}$ probably also belongs to the first class (with the likely structure $\mathrm{Fm} \overline{3}$ or $\mathrm{Fm} \overline{3} \mathrm{~m}$ ) and its $T_{c}=29.6 \mathrm{~K}$ is essentially what one would expect from the large lattice parameter $a=14.473 \AA$ (Zhou et al., 1993). $\mathrm{Cs}_{3} \mathrm{C}_{60}$ has a different (noncubic) structure and a different pressure dependence than the other $\mathrm{A}_{3} \mathrm{C}_{60}$ compounds and it should not be considered as a memeber of the first class. The maximum $T_{c}$ found for $\mathrm{Cs}_{3} \mathrm{C}_{60}$ is $40 \mathrm{~K}$ (Palstra et al., 1995). Another interesting system is $\mathrm{NH}_{3} \mathrm{~K}_{3} \mathrm{C}_{60}$ which like $\mathrm{K}_{3} \mathrm{C}_{60}$ has three electrons in the $t_{1 u}$ orbital but an orthorhombic distortion of the fcc lattice (Rosseinsky et al., 1993; Zhou et al., 1995). At ambient pressure this system shows no superconductivity above $4 \mathrm{~K}$ and it is possibly an insulator. Under pressure, however, superconductivity occurs with the maximum observed $T_{c}$ being $28 \mathrm{~K}$. It had been suggested that only cubic fullerides can be superconductors, but $\mathrm{Cs}_{3} \mathrm{C}_{60}$ and $\mathrm{NH}_{3} \mathrm{~K}_{3} \mathrm{C}_{60}$ under pressure now provide examples of noncubic superconducting fullerides. These compounds nevertheless raise interesting questions about the role of the deviation from cubic symmetry, since the application of pressure reduces this symmetry lowering and increases $T_{c}$ (Palstra et al., 1995; Zhou et al., 1995). Alternatively, 
the closeness to a Mott transition may be important for the pressure dependence (Palstra et al., 1995; Zhou et al., 1995).

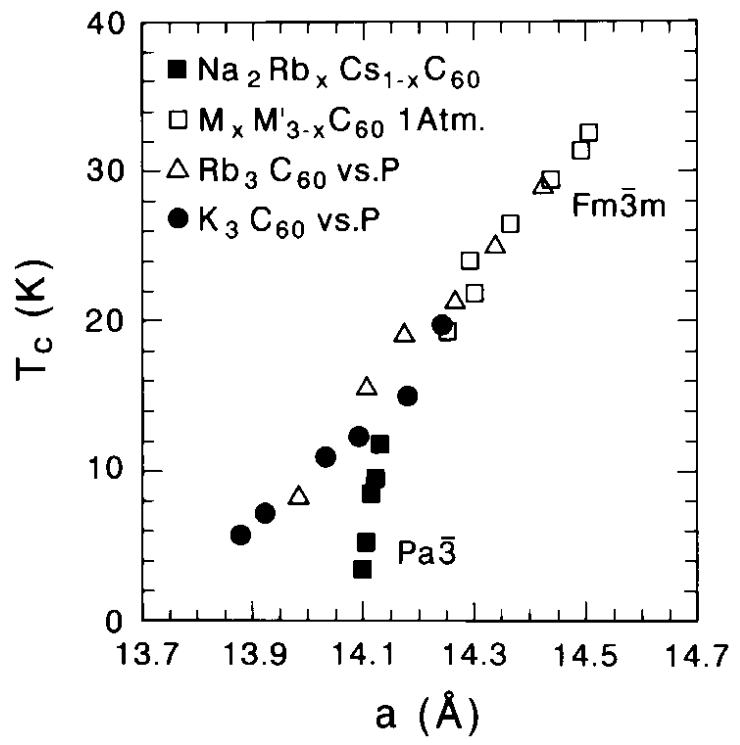

FIG. 3. $T_{c}$ as a function of the lattice parameter $a$ for the $\mathrm{Fm} \overline{3} \mathrm{~m}$ (triangles, circles and open squares) and $\mathrm{Pa} \overline{3}$ (filled squares) superconductors. For $\mathrm{K}_{3} \mathrm{C}_{60}$ and $\mathrm{Rb}_{3} \mathrm{C}_{60}$ the lattice parameter was varied by changing the pressure, while for $\mathrm{M}_{x} \mathrm{M}_{3-x}^{\prime} \mathrm{C}_{60}$ (with $\mathrm{M}, \mathrm{M}^{\prime}=\mathrm{K}, \mathrm{Rb}, \mathrm{Cs}$ ) the lattice parameter was varied by changing the composition. (After Yildirim et al. 1994).

Yildirim et al. (1996a) have studied $\mathrm{Na}_{2} \mathrm{Cs}_{x} \mathrm{C}_{60}$ for $x=0.25,0.50$ and 0.75 . The values of $T_{c}$ found for these compounds are shown in Fig. 4 as a function of the expected valence $n=2+x$. It is found that $T_{c}$ drops quickly as the valence is reduced. For $n=2.5$ no superconductivity was observed above $T=0.5 \mathrm{~K}$, while the systems are believed to be metallic down to $n=2.25$ (Yildirim et al., 1996a).

Yildirim et al. $(1995 ; 1996 \mathrm{ab})$ studied $\mathrm{Rb}_{3-x} \mathrm{Ba}_{x} \mathrm{C}_{60}$ for $x=0.25,1$ and 2. Based on Raman data and theoretical considerations, they concluded that the $\mathrm{C}_{60}$ valence is $n=3+x$ and that $\mathrm{Ba}$ is essentially completely ionized. The results for $T_{c}$ are shown in Fig. 4. $T_{c}$ drops rapidly with $x$ and for $x=1(n=4)$ and $x=2(n=5)$ no superconductivity was observed above $T=0.5 \mathrm{~K}$. These compounds show a very small Pauli susceptibility, and it was tentatively concluded that they are weakly metallic (Yildirim et al., 1996b). Fig. 4 suggests a rapid drop of $T_{c}$ when the $\mathrm{C}_{60}$ valence deviates from 3 . The reduction for $n>3$, but not for $n<3$ (see Sec. VIIA.2), may be explainable by the reduction of the density of states with increasing filling, taking the merohedral disorder into account (Yildirim et al., 1995). The reduction of $T_{c}$ as $n$ is reduced below 3 seems not be understood. Fig. 4 furthermore appears to be inconsistent with the conclusion that stoichiometric $\mathrm{A}_{3} \mathrm{C}_{60}$ is an insulator (Lof et al., 1992). In such a case one might expect $T_{c}$ to increase when the occupancy of the $t_{1 u}$ orbital moves away from integer values, in analogy with the high $T_{c}$ superconductors, while Fig. 4 shows the opposite trend.

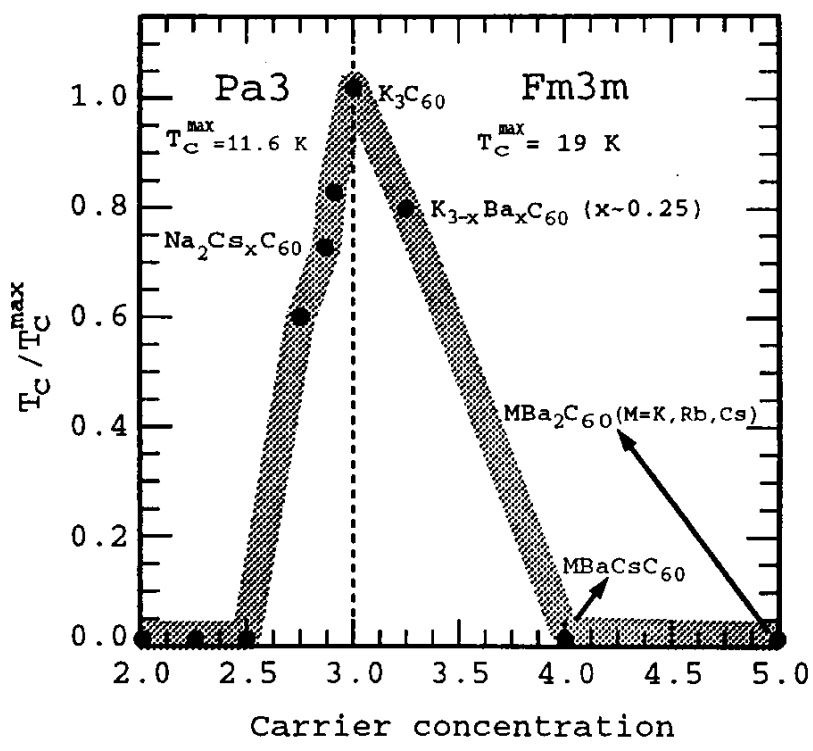

FIG. 4. $T_{c}$ as a function of $\mathrm{C}_{60}$ valence $n$ for the $\mathrm{Na}_{2} \mathrm{Cs}_{x} \mathrm{C}_{60}$ $(x \leq 1, n \leq 3)$ and $\mathrm{K}_{3-x} \mathrm{Ba}_{x} \mathrm{C}_{60}(n \geq 3)$ compounds. $T_{c}$ is scaled by the $T_{c}\left(T_{c}^{\max }\right)$ of the end members $\mathrm{Na}_{2} \mathrm{CsC}_{60}$ and $\mathrm{K}_{3} \mathrm{C}_{60}$. The heavy line is a guide for the eye. Observe the different lattice structures. (After Yildirim et al., 1996a).

In the fourth group the measured transition temperatures are $8.4 \mathrm{~K}\left(\mathrm{Ca}_{x} \mathrm{C}_{60}\right), 4 \mathrm{~K}\left(\mathrm{Sr}_{x} \mathrm{C}_{60}\right)$ and $7 \mathrm{~K}$ $\left(\mathrm{Ba}_{x} \mathrm{C}_{60}\right)$.

Recently, superconductivity has been found in $\mathrm{RE}_{x} \mathrm{C}_{60}$, where $\mathrm{RE}$ is a rare earth atom. Özdas et al. (1995) discovered that $\mathrm{Yb}_{2.75} \mathrm{C}_{60}$ is superconducting with $T_{c}=6 \mathrm{~K}$. This system has a slight orthorhombic distortion of the $\mathrm{Pa} \overline{3}$ structure and the structure is Pcab. Based on EXAFS measurements it was concluded that $\mathrm{Yb}$ is divalent, which would imply the $\mathrm{C}_{60}$ valence 5.5 (Özdas et al., 1995). Chen and Roth (1995) found superconductivity in $\mathrm{Sm}_{x} \mathrm{C}_{60}(x<3)$ with $T_{c}=8 \mathrm{~K}$.

It is also interesting to observe that $\mathrm{Li}_{2} \mathrm{AC}_{60}$ is not superconducting (Hirosawa et al., 1994). This system has a different structure than $\mathrm{Na}_{2} A \mathrm{C}_{60}$ and $\mathrm{A}_{3} \mathrm{C}_{60}$. It has the space group $\mathrm{Fm} \overline{3} \mathrm{~m}$, but with an orientational state of $\mathrm{C}_{60}$ which is best modeled as a quasi-spherical unit (Hirosawa et al., 1994).

\section{B. Superconductivity gap}

The superconductivity gap $\Delta$ is of great interest, since a value of the reduced gap $2 \Delta / T_{c}$ which is substantially 
larger than the BCS value (3.53) indicates that strongcoupling effects are important. For intermediate coupling, it is found that (Mitrovic et al., 1984) (see, also Carbotte (1990) for a discussion)

$$
\frac{2 \Delta}{T_{c}}=3.53\left[1+12.5\left(\frac{T_{c}}{\omega_{l n}}\right)^{2} \ln \left(\frac{\omega_{l n}}{2 T_{c}}\right)\right]
$$

where $\omega_{l n}$ is the logarithmic average phonon frequency. This formula says that the deviation from the BCS result is small if $T_{c} / \omega_{l n}<<1$. An early measurement of $\Delta$ was performed using point contact tunneling in STM, giving $2 \Delta / T_{c}=5.3$ for $\mathrm{Rb}_{3} \mathrm{C}_{60}$ (Zhang et al., 1991b). Later STM measurements on single crystals have given reduced gaps $2 \Delta / T_{c}=5.4$ (Jess et al., 1994) and $2 \Delta / T_{c}=2.0-4.0$ (Jess et al., 1996). The variations in $2 \Delta / T_{c}$ were ascribed to variations in the local stoichiometry at the tip position. From measurements using NMR the values 3.0 for $\mathrm{K}_{3} \mathrm{C}_{60}$ and 4.1 for $\mathrm{Rb}_{3} \mathrm{C}_{60}$ (Tycko et al., 1992), 4.3 for $\mathrm{K}_{3} \mathrm{C}_{60}$ (Sasaki et al., 1994) and $3.4 \pm 0.2 \mathrm{~K}_{3} \mathrm{C}_{60}$ (Auban-Senzier et al., 1993) were deduced. Recent NMR measurements were found to be in good agreement with the BCS value for the gap (Stenger et al., 1995). Using muon spin relaxation, Kiefl et al. (1993) obtained the value 3.6 for $\mathrm{Rb}_{3} \mathrm{C}_{60}$. Recent optical measurements have given the values 3.44 for $\mathrm{K}_{3} \mathrm{C}_{60}$ and 3.45 for $\mathrm{Rb}_{3} \mathrm{C}_{60}$ (Degiorgi et al., 1994, Degiorgi, 1995). Finally, the reduced gap was measured in photoemission, giving the value 4.1 for $\mathrm{Rb}_{3} \mathrm{C}_{60}$ ( $\mathrm{Gu}$ et al., 1994). We note that the data obtained from different experiments show a substantial variation. Most recent results, however, tend to scatter around the weak-coupling limit and are consistent with this limit, if a reasonable uncertainty is assumed for these experiments.

\section{Isotope effect}

The isotope effect may provide interesting information about the mechanism for superconductivity. In the BCS theory for a system with only one type of ions with the mass $M$, the transition temperature behaves as $T_{c} \sim M^{-\alpha}$, where $\alpha=0.5$. For the $\mathrm{A}_{3} \mathrm{C}_{60}$ compounds, the $\mathrm{C}_{60}$ phonons are expected to be the important ones, and one is therefore interested in the isotope effect when ${ }^{12} C$ is substituted by ${ }^{13} C$. For the case of complete (99\%) substitution, it was found that $\alpha=0.30 \pm 0.06$ for $\mathrm{K}_{3} \mathrm{C}_{60}$ (Chen and Lieber, 1992) and $\alpha=0.30 \pm 0.05$ for $\mathrm{Rb}_{3} \mathrm{C}_{60}$ (Chen and Lieber, 1993). Interesting and contradictory results have been obtained for incomplete substitution. Chen and Lieber (1993) found that for a single peak mass distribution of $\mathrm{C}_{60}$ molecules $\left(\mathrm{Rb}_{3}\left({ }^{13} \mathrm{C}_{0.55}{ }^{12} \mathrm{C}_{0.45}\right)_{60}\right)$, the value of $\alpha$ was consistent with the result for complete substitution. On the other hand, they found that a two peak mass distribution $\left(\mathrm{Rb}_{3}\left({ }^{13} \mathrm{C}_{60}\right)_{0.5}\left({ }^{12} \mathrm{C}_{60}\right)_{0.5}\right)$ gave a much larger value of $\alpha(\sim 0.8)$. A value of $\alpha$ which is larger than 0.5 is unusual, but it was also observed by three other groups. Ebbesen et al. (1992a) obtained $\alpha=1.4 \pm 0.5$ for $\mathrm{Rb}_{3} \mathrm{C}_{60}$ (33\% substitution), Zakhidov et al. (1992) obtained $\alpha=(1.2-1.43) \pm 0.2$ for $\mathrm{K}_{3} \mathrm{C}_{60}$ and $\alpha=(2-2.25) \pm 0.25$ for $\mathrm{Rb}_{3} \mathrm{C}_{60}(60 \%$ substitution $)$ and Auban-Senzier et al. (1993) obtained $\alpha=1.45 \pm 0.3$ for $82 \%$ substitution. However, these results were observed for distributions of $\mathrm{C}_{60}$ masses with essentially only one peak, while the $\alpha>0.5$ was obtained by Chen and Lieber (1993) for a two-peak distribution. Finally Ramirez et al. (1992a) obtained $\alpha=0.37 \pm 0.05$ for mass distribution of $15 \%{ }^{12} \mathrm{C}_{60}$ and the rest $\left({ }^{13} \mathrm{C}_{0.9}{ }^{12} \mathrm{C}_{0.1}\right)_{60}$. For the partially substituted compounds, the experimental situation thus appears to be unclear. The results are summarized in Fig. 5. The theoretical considerations have so far, however, been made for completely substituted systems, where $\alpha=0.3$ should be the best available experimental result. The theoretical treatment of partially substituted compounds remains a challenging problem.

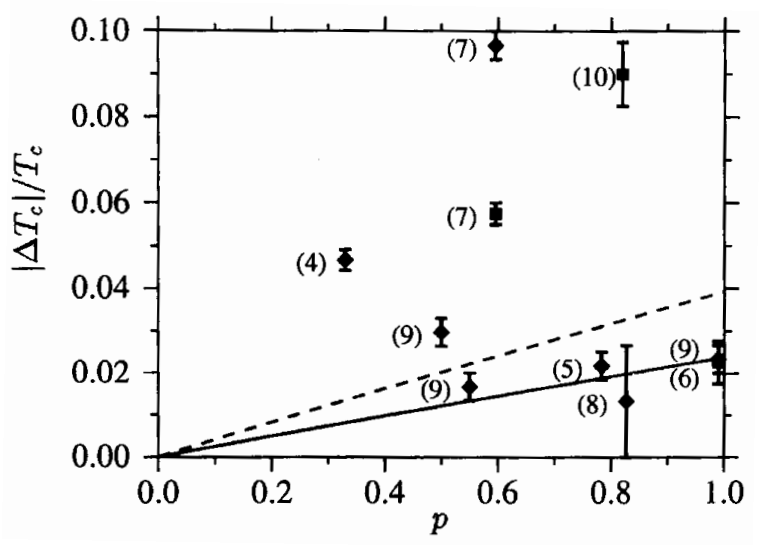

FIG. 5. The isotope shift $\Delta T_{c}$ of $T_{c}$ for $\mathrm{K}_{3} \mathrm{C}_{60}$ (squares) and $\mathrm{Rb}_{3} \mathrm{C}_{60}$ (diamonds) as a function of the fraction $p$ of the substitution $\left(p=1\right.$ completely $\left.{ }^{13} C\right)$. The dashed line shows the BCS result $\alpha=0.5$ and the full line $\alpha=0.3$. The results are obtained from (4) Ebbesen et al. (1992), (5) Ramirez et al. (1992a), (6) Chen and Liber (1992), (7) Zakhidov et al. (1992), (8) Bethune et al. (1992), (9) Chen and Lieber (1993), (10) Auban-Senzier et al. (1993). (After Deaven and Rokhsar, 1993).

The isotope effect with respect to the alkali dopants have also been studied. It was found that within the experimental uncertainty there is no isotope effect (Ebbesen et al. 1992b; Burk et al. 1994ab). This is consistent with the experimental result that applying pressure to $\mathrm{Rb}_{3} \mathrm{C}_{60}$ so that the lattice parameter is reduced to that of $\mathrm{K}_{3} \mathrm{C}_{60}$ leads to about the same $T_{c}$ for both systems (Fleming et al. 1991). This strongly suggests that the alkali ions have a very weak influence on $T_{c}$ except for the indirect influence via the lattice parameter. 


\section{Other properties}

$\mathrm{A}_{3} \mathrm{C}_{60}$ is a type II superconductor. The lower critical field $H_{c 1}$ has been measured to be 130 Oe (Holczer et al., 1991b) for $\mathrm{K}_{3} \mathrm{C}_{60}$ and 120 Oe (Sparn et al, 1992) and $60 \pm 5$ Oe (Ramirez et al., 1994) for $\mathrm{Rb}_{3} \mathrm{C}_{60}$. To estimate the penetration depth $\lambda(0)$, one can now use the relationship with $H_{c 1} \lambda(0)$ (Tinkham, 1975)

$$
H_{c 1}=\frac{\phi_{0}}{4 \pi \lambda(0)^{2}} \ln \left(\frac{\lambda(0)}{\xi_{G L}}\right),
$$

where $\phi_{0}=h c / e$ and $\xi_{G L}$ is the Ginzburg-Landau coherence length. Putting $\xi_{G L}=30 \AA$, which is reasonable in view of the results below, one obtains the values $\lambda(0)=2500 \AA$ for $\mathrm{K}_{3} \mathrm{C}_{60}$ and 2600 and $3700 \AA$ for $\mathrm{Rb}_{3} \mathrm{C}_{60}$. The penetration depth has also been estimated from $\mu \mathrm{SR}$ with the results $4800 \AA$ for $\mathrm{K}_{3} \mathrm{C}_{60}$ and $4200 \AA$ for $\mathrm{Rb}_{3} \mathrm{C}_{60}$ (Uemura et al., 1991; 1993), and from the optical conductivity with the result $8000 \AA$ for both $\mathrm{K}_{3} \mathrm{C}_{60}$ and $\mathrm{Rb}_{3} \mathrm{C}_{60}$ (Degiorgi et al., 1992) and from NMR data giving $6000 \AA$ for $\mathrm{K}_{3} \mathrm{C}_{60}$ and $4600 \AA$ for $\mathrm{Rb}_{3} \mathrm{C}_{60}$ (Tycko et al., 1992). Uemura et al. (1991) also deduced the temperature dependence of penetration depth $\lambda(T)$. They could fit these results with the formula $\left[1-\left(T / T_{c}\right)^{\alpha}\right]$, with $\alpha=3.2$. From this they concluded that $\mathrm{K}_{3} \mathrm{C}_{60}$ probably is an isotropic s-wave superconductor, as expected. Based on values for $\lambda(0)$ and $\xi$, Uemura et al. (1991) also estimated an "effective" Fermi temperature $T_{F}^{e f f}$. Combining their small value $(470 \mathrm{~K})$ for $T_{F}^{e f f}$ with $T_{c}$ in a $T_{c}$ vs. $T_{F}^{e f f}$ plot, they found that the $\mathrm{A}_{3} \mathrm{C}_{60}$ belongs to a group of "exotic" superconductors, including the High $T_{c}$ cuprates and the organic BEDT systems.

The upper critical field $H_{c 2}(0)$ is of interest, since it allows an estimate of the coherence length via (Tinkham, 1975)

$$
H_{c 2}(0)=\frac{\phi_{0}}{2 \pi \xi_{G L}}
$$

Several groups have measured $H_{c 2}^{\prime} \equiv d H_{c 2} / d T$ and then obtained the value of $H_{c 2}(0)$ from the WertheimerHelfand-Hohenberg (Wertheimer et al., 1966) theory. Based on this, $\xi_{G L}$ was deduced to be $26 \AA$ for $\mathrm{K}_{3} \mathrm{C}_{60}$ (Holczer et al., 1991b) and $20 \AA$ for $\mathrm{Rb}_{3} \mathrm{C}_{60}$ (Sparn et al., 1992) from dc-magnetization. The high-field susceptibility gave the values $29 \AA$ to $33 \AA$ (Boebinger et al., 1992) and $35 \AA$ (Holczer and Whetten, 1992) for $\mathrm{K}_{3} \mathrm{C}_{60}$ and $30 \AA$ (Holczer and Whetten, 1992) for $\mathrm{Rb}_{3} \mathrm{C}_{60}$. From the ratio of $\xi_{G L} / \lambda$ it is clear that $A_{3} \mathrm{C}_{60}$ is a type II superconductor. The magnetic properties of the $\mathrm{C}_{60}$ superconductors have been reviewed by Buntar and Weber (1996).

The jump in the specific heat at $T_{c}$ was estimated by Ramirez et al. (1992) to be $\Delta C=68 \pm 13 \mathrm{~mJ} / \mathrm{mole}^{2} \mathrm{~K}^{2}$ for $\mathrm{K}_{3} \mathrm{C}_{60}$ and by Burkhart and Meingast (1996) to be
$64 \pm 14$ and $75 \pm 14 \mathrm{~mJ} / \mathrm{mole}-\mathrm{K}^{2}$ for $\mathrm{K}_{3} \mathrm{C}_{60}$ and $\mathrm{Rb}_{3} \mathrm{C}_{60}$, respectively. The Hebel-Slichter peak was not seen in early NMR measurements (Tycko et al., (1992), but has been seen in $\mu \mathrm{SR}$ (Kiefl et al., (1993)) and more recent NMR (Stenger et al., 1995) measurements. The vertex glass and vertex fluid phase diagram of $\mathrm{A}_{3} \mathrm{C}_{60}$ has been studied by Lin et al. (1994).

In Table II we have summarized some superconducting parameters. The table has mainly been extracted from Ramirez (1994), but it has been partly updated in the view of newer experiments.

TABLE II. Superconducting parameters for $\mathrm{K}_{3} \mathrm{C}_{60}$ and $\mathrm{Rb}_{3} \mathrm{C}_{60}$. (Mainly after Ramirez (1994)).

\begin{tabular}{lll}
\hline \hline Property & $\mathrm{K}_{3} \mathrm{C}_{60}$ & $\mathrm{Rb}_{3} \mathrm{C}_{60}$ \\
\hline$T_{c}$ & $19.5 \mathrm{~K}$ & $29.5 \mathrm{~K}$ \\
$\lambda(\mu \mathrm{SR})$ & $4800 \pm 200 \AA$ & $4200 \AA$ \\
$\lambda$ (optical) & $8000 \pm 500 \AA$ & $8000 \pm 500 \AA$ \\
$\lambda(\mathrm{NMR})$ & $6000 \AA$ & $4600 \AA$ \\
$\xi_{0}\left(\chi_{a c}\right)$ & $29-35 \AA$ & $30 \AA$ \\
$\Delta C / T_{c}$ & $64 \pm 14 \mathrm{~mJ} / \mathrm{mole}^{2} \mathrm{~K}^{2}$ & $75 \pm 14 \mathrm{~mJ} / \mathrm{mole}-\mathrm{K}^{2}$ \\
$2 \Delta / k_{b} T_{c}$ (NMR) & 3.0 & 4.1 \\
$2 \Delta / k_{b} T_{c}$ (optical) & 3.44 & 3.45 \\
$2 \Delta / k_{b} T_{c}$ ( $\left.\mu \mathrm{SR}\right)$ & & $3.6 \pm 0.3$ \\
$2 \Delta / k_{b} T_{c}$ (tunneling) & $5.3 \pm 0.2$ & $5.2 \pm 0.3$ \\
$2 \Delta / k_{b} T_{c}$ (tunneling) & $5.4,2.0-4.0$ \\
\hline \hline
\end{tabular}

\section{PHONONS AND ELECTRON-PHONON COUPLING}

The phonons and the electron-phonon interaction are very important for the properties of alkali-fullerides. The electron-phonon interaction is usually assumed to cause the superconductivity. It should, however, also be important for the transport properties in general, and may also play a substantial role for other electronic properties.

The phonons of alkali-fullerides can be divided in subgroups, which reflect the molecular nature of the solid. The highest modes are due to intramolecular vibrations, with energies in the range $273-1575 \mathrm{~cm}^{-1}$ (0.034-0.195 $\mathrm{eV}$ ) for an undoped $\mathrm{C}_{60}$ solid (Bethune et al., 1991). The intermolecular phonons occur at substantially lower energies in the range up to about $140 \mathrm{~cm}^{-1}(17 \mathrm{meV})$ (Prassides et al., 1991; Pintschovius, 1996). These modes contain both $\mathrm{C}_{60}-\mathrm{C}_{60}$ and $\mathrm{A}-\mathrm{C}_{60}(\mathrm{~A}=\mathrm{K}, \mathrm{Rb})$ vibrations. Finally, there are librations, which occur at an energy of about 4-5 meV (Christides et al., 1992; Schober et al., 1994). These various phonons are schematically illustrated in Fig. 6. It is believed that the intramolecular modes play the main role for the superconductivity. We therefore focus on these modes, but also the other modes are discussed. 


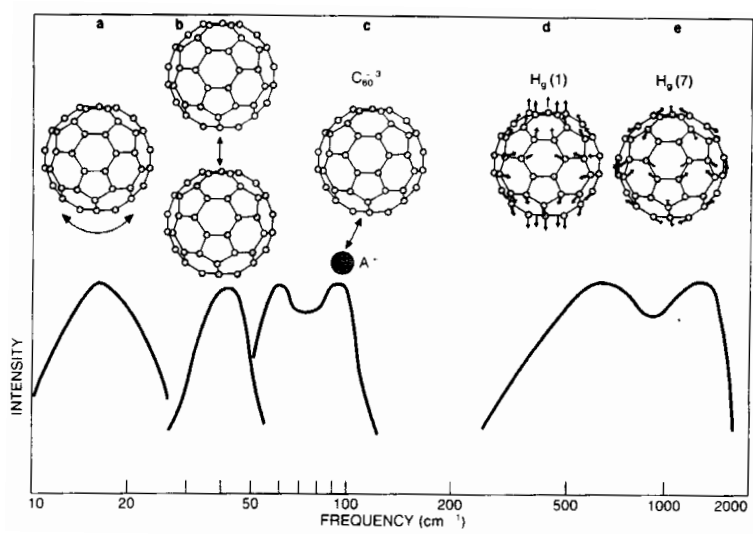

FIG. 6. Schematic representation of various phonons in $\mathrm{A}_{3} \mathrm{C}_{60}$ compounds. The figure shows (a) librations, (b) optic $\mathrm{C}_{60}-\mathrm{C}_{60}$ phonons, (c) A- $\mathrm{C}_{60}$ phonons and (d)-(e) intramolecular $\mathrm{H}_{g}$ modes. The figure indicates the radial and tangential character of the low-lying and and high-lying $\mathrm{H}_{g}$ modes, respectively. (After Hebard, 1992).

\section{A. Intramolecular modes}

For the intramolecular phonons, the effects of the interactions between the $\mathrm{C}_{60}$ molecules are small. These phonons can therefore be approximately classified by the icosahedral point group. In alkali-fullerides, the $t_{1 u}$ band is (partly) populated. The coupling of the phonons to this band is therefore of particular interest. From symmetry arguments, it follows that only the two phonons with $\mathrm{A}_{g}$ and the eight phonons with $\mathrm{H}_{g}$ symmetry can couple (Varma et al., 1991; Lannoo et al., 1991). We therefore focus our interest on these modes.

\section{Calculations of phonon frequencies}

There have been a number of calculations of the phonon frequencies. In Table III we show the results of calculations using the semi-empirical MNDO method (Varma et al., 1991), an empirical force-constant model (Jishi et al., 1992) and the ab initio density functional method (Hohenberg and Kohn, 1964; Kohn and Sham, 1965 ) in the local density approximation (LDA) but using different numerical methods ( Antropov et al., 1993b; Quong et al., 1993; Faulhaber et al., 1993). These calculations were all for a free $\mathrm{C}_{60}$ molecule, except the one by Antropov et al., (1993b), which was for $\mathrm{K}_{3} \mathrm{C}_{60}$ in the socalled uni-directional structure, where all $\mathrm{C}_{60}$ molecules have the same orientation (where a cubic axis and a twofold molecular axis coincide). These calculations can be considered as representative for the large number of calculations that have been performed (see also, e.g., Negri et al., 1988; Adams et al., 1991; Kohanoff et al., 1992; Jones et al., 1992; Onida and Benedek, 1992; Bohnen et al., 1995). These results are compared with experimental results obtained from Raman scattering (Bethune et al., 1991). We can see that the best empirical and ab initio calculations have errors of the order one per cent. The small errors in, for instance, the calculations of Quong et al., (1993) suggests that the LDA errors for the frequencies are small.

Bohnen et al. (1995) have calculated the changes in the phonon frequencies when going from solid $\mathrm{C}_{60}$ to $\mathrm{K}_{3} \mathrm{C}_{60}$, and found a softening of the modes, in particular the high-frequency ones.

TABLE III. Phonon frequencies (in $\mathrm{cm}^{-1}$ ) for the $\mathrm{H}_{g}$ and $\mathrm{A}_{g}$ modes. Different theoretical calculations are compared with experimental results for undoped solid $\mathrm{C}_{60}$.

\begin{tabular}{lcccccc}
\hline \hline Mode & \multicolumn{5}{c}{ Energy $^{\mathrm{c}}$} \\
& Varma $^{\mathrm{a}}$ & Jishi $^{\mathrm{b}}$ & Antropov $^{\mathrm{c}}$ & Quong $^{\mathrm{d}}$ & Faulhaber $^{\mathrm{e}}$ & Exp. $^{\mathrm{f}}$ \\
\hline $\mathrm{H}_{g}(8)$ & 1721 & 1575 & 1462 & 1576 & 1567 & 1575 \\
$\mathrm{H}_{g}(7)$ & 1596 & 1401 & 1387 & 1443 & 1425 & 1428 \\
$\mathrm{H}_{g}(6)$ & 1406 & 1217 & 1290 & 1244 & 1200 & 1250 \\
$\mathrm{H}_{g}(5)$ & 1260 & 1102 & 1091 & 1093 & 1067 & 1099 \\
$\mathrm{H}_{g}(4)$ & 924 & 788 & 785 & 767 & 750 & 774 \\
$\mathrm{H}_{g}(3)$ & 711 & 708 & 753 & 727 & 640 & 710 \\
$\mathrm{H}_{g}(2)$ & 447 & 439 & 454 & 439 & 421 & 437 \\
$\mathrm{H}_{g}(1)$ & 263 & 269 & 281 & 258 & 249 & 273 \\
$\mathrm{~A}_{g}(2)$ & & 1468 & 1463 & 1499 & 1493 & 1470 \\
$\mathrm{~A}_{g}(1)$ & & 492 & 458 & 478 & 459 & 496 \\
\hline \hline
\end{tabular}

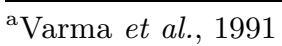

b Jishi et al., 1992

${ }^{\mathrm{c}}$ Antropov et al., 1993

${ }^{\mathrm{d}}$ Quong et al., 1993

${ }^{\mathrm{e}}$ Faulhaber et al., 1993

${ }^{\mathrm{f}}$ Bethune et al., 1991

The four highest modes are primarily made up of vibrations tangentially to the $\mathrm{C}_{60}$ surface, while the three lowest modes have mainly radial character (Schluter et al., 1992ab; Antropov et al., 1993b), as indicated in Fig. 6 . The reason is that the molecule is much stiffer against tangential than radial distortions, since the former require larger bond length changes. The phonon frequencies are high, extending up to about $0.2 \mathrm{eV}$. This is due to both the light mass of carbon and to the stiffness of the $\mathrm{C}-\mathrm{C}$ bond.

\section{Calculations of electron-phonon coupling}

The electron-phonon coupling plays a crucial role in the theory of superconductivity. We introduce the dimensionless coupling to the phonon mode $\nu$ (Rainer, 1986)

$$
\begin{array}{r}
\lambda_{\nu}=\frac{2}{N(0)} \sum_{\mathbf{q}} \frac{1}{\omega_{\nu \mathbf{q}}} \sum_{n, m, \mathbf{k}}\left|g_{n \mathbf{k}, m(\mathbf{k}+\mathbf{q})}(\nu)\right|^{2} \\
\times \delta\left(\varepsilon_{n \mathbf{k}}\right) \delta\left(\varepsilon_{m(\mathbf{k}+\mathbf{q})}-\varepsilon_{n \mathbf{k}}-\omega_{\nu \mathbf{q}}\right),
\end{array}
$$


where $N(0)$ is the electron density of states per spin at the Fermi energy and $\omega_{\nu \mathbf{q}}$ is the energy of the $\nu$ th phonon with the wave vector q. The energy of the $n$th electronic band with the wave vector $\mathbf{k}$ is $\varepsilon_{n \mathbf{k}}$. Finally, $g_{n \mathbf{k}, m(\mathbf{k}+\mathbf{q})}(\nu)$ is the matrix element between the two electronic state $n \mathbf{k}$ and $m(\mathbf{k}+\mathbf{q})$ of the potential created when a phonon $\nu \mathbf{q}$ is excited. Thus it follows

$$
g_{n \mathbf{k}, m(\mathbf{k}+\mathbf{q})}(\nu)=\left\langle n \mathbf{k}\left|\Delta V_{\nu \mathbf{q}}\right| m(\mathbf{k}+\mathbf{q})\right\rangle \frac{1}{\sqrt{2 M \omega_{\nu \mathbf{q}}}},
$$

where $\Delta V_{\nu \mathbf{k}}$ is the induced potential per unit displacement along the phonon normal coordinate, $\sqrt{2 M \omega_{\nu \mathbf{q}}}$ is the phonon amplitude and $M$ is the reduced mass.

The calculation of $\lambda$ requires a double sum over a Brillouin zone of a quantity $(g)$ which is very time consuming to calculate. In general, an accurate calculation of $\lambda$ is therefore very complicated. For the intramolecular modes in the fullerides, important simplifications follow from the large difference between the intramolecular $\left(E_{I}\right)$ and intermolecular $(W)$ energy scales (Lannoo et al., 1991). One can then assume that the phonon eigenvalues and eigenvectors are independent of $\mathbf{q}$. If in addition we assume that $W<<E_{I}$ in the calculation of $g_{n \mathbf{k}, m(\mathbf{k}+\mathbf{q})}(\nu)$, but keep the full structure of $|n \mathbf{k}\rangle$, the complicated Brillouin zone sums can be performed analytically, and the problem is essentially reduced to a molecular problem (Lannoo et al., 1991). The accuracy of these assumptions has been tested for a simple tightbinding model, where it was possible to perform the Brillouin zone summations (Antropov et al., 1993b). It was found that the simplified calculation agreed fairly well with the full calculation, justifying the assumptions of Lannoo et al. (1991).

Thus it is sufficient to calculate the shift $\Delta \varepsilon_{\nu \alpha}$ of the $t_{1 u}$ level $\alpha$ for a free $\mathrm{C}_{60}$ molecule per unit displacement of the $\nu$ th phonon coordinate. One then finds that

$$
\lambda \sim N(0) \sum_{\nu \alpha} \frac{\Delta \varepsilon_{\nu \alpha}^{2}}{\omega_{\nu}^{2}}
$$

This gives a molecular specific quantity which must then be multiplied by the density of states $N(0)$, which is determined by the intermolecular hopping. The molecular calculation therefore gives a value for $\lambda_{\nu} / N(0)$, and it is appropriate to compare this quantity from different calculations. We observe that the quantity $\lambda_{\nu} / N(0)$ grows with the intramolecular energy scale, while $N(0)$ is inversely proportional to the intermolecular hopping. The large difference between these two energy scales therefore favors a large value of $\lambda$ (Schluter et al., 1992a). It is also clear that as the lattice parameter increases, the overlap between the $\mathrm{C}_{60}$ molecules is decreased, reducing the band width. This leads to a larger density of states $N(0)$ and a larger $\lambda$, explaining the increase in $T_{c}$ with lattice parameter (Rosseinsky et al., 1991; Flemming et al., 1991; Tanigaki et al., 1993; Ramirez, 1994) (see, also Fig. 3).

We notice that the results are quite sensitive to the phonon eigenvectors, and small changes in the eigenvectors can lead to large shifts of the coupling strength between different phonons. One can show, however, that $\sum_{\nu \alpha}\left(\Delta \varepsilon_{\nu \alpha}\right)^{2}$ is independent of the phonon eigenvectors (Antropov et al., 1993b). A shift of coupling strength between phonons with similar energies therefore has a small effect on the total value of $\lambda$, while a shift between phonons of very different energies can dramatically change the total $\lambda$ due to the energy denominator in Eq. (7). For instance, in the calculation of Antropov et al. (1993b), mixing $5 \%$ of the weight $(\sqrt{0.05})$ of the seventh eigenvector into the eighth and vice versa changes the couplings 0.020 and 0.022 in Table IV to 0.010 and 0.030 , i.e. a small change in the total coupling. The same mixing between the second and the eighth eigenvectors changes the couplings 0.006 and 0.022 to 0.033 and 0.019 , i.e. a large change of the total coupling.

TABLE IV. The partial electron-phonon coupling constants $\lambda_{\nu} / N(0)$ (in eV) according to different theoretical calculations. The energies (in $\mathrm{cm}^{-1}$ ) of the modes for the undoped system are shown.

\begin{tabular}{lccccc}
\hline \hline Mode & Energy & \multicolumn{4}{c}{$\lambda_{\nu} / N(0)$} \\
& & Varma $^{\mathrm{a}}$ & Schluter $^{\mathrm{b}}$ & Antropov $^{\mathrm{c}}$ & Faulhaber $^{\mathrm{d}}$ \\
\hline $\mathrm{H}_{g}(8)$ & 1575 & .011 & .009 & .022 & .009 \\
$\mathrm{H}_{g}(7)$ & 1428 & .034 & .013 & .020 & .015 \\
$\mathrm{H}_{g}(6)$ & 1250 & .000 & .003 & .008 & .002 \\
$\mathrm{H}_{g}(5)$ & 1099 & .006 & .001 & .003 & .002 \\
$\mathrm{H}_{g}(4)$ & 774 & .000 & .007 & .003 & .010 \\
$\mathrm{H}_{g}(3)$ & 710 & .001 & .004 & .003 & .001 \\
$\mathrm{H}_{g}(2)$ & 437 & .001 & .007 & .006 & .010 \\
$\mathrm{H}_{g}(1)$ & 273 & .003 & .008 & .003 & .001 \\
$\sum \mathrm{H}_{g}$ & & .056 & .052 & .068 & .049 \\
\hline \hline
\end{tabular}

a Varma et al., 1991

${ }^{\mathrm{b}}$ Schluter et al., 1992b

${ }^{\mathrm{c}}$ Antropov et al., 1993b

${ }^{\mathrm{d}}$ Faulhaber et al., 1993

In Table IV we show some typical results of calculations of $\lambda / N(0)$. The calculation of Varma et al. (1991) was based on the semi-empirical MNDO method. The calculation of Schluter et al. (1992b) used an empirical bond charge model for the phonons and a LDA calculation for the electronic part. The work of Antropov et al. (1993b) and Faulhaber et al. (1993) used ab initio LDA calculations both to generate the phonons and to calculate their interactions with the electrons. There are several other calculations. Mazin et al. (1992) performed an ab initio LDA calculation in the ASA-LMTO formalism and used an empirical phonon model to obtain $\lambda / N(0)=0.037 \mathrm{eV}$. Coulon et al. (1992) performed an $a b$ initio LDA calculation using a pseudopotential Gaussian method and found $\lambda / N(0) \geq 0.04 \mathrm{eV}$. Asai and 
Kawaguchi (1992) obtained $\lambda / N(0)=0.070 \mathrm{eV}$. The coupling to the $\mathrm{A}_{g}(2)$ mode for a free molecule was estimated by Stollhoff (1991) to be $\lambda_{A_{g}(2)} / N(0)=0.0025 \mathrm{eV}$ and by Scherrer and Stollhoff (1993) to be $\lambda_{A_{g}(2)} / N(0)=0.01$ $\mathrm{eV}$. Relatively small values for the coupling to the $\mathrm{A}_{g}(2)$ mode were also deduced by Schlüter et al.

$\left(\lambda_{A_{g}(2)} / N(0)=0.005 \mathrm{eV}\right)$, Antropov et al.

$\left(\lambda_{A_{g}(2)} / N(0)=0.009 \mathrm{eV}\right)$ and Pickett et al.

$\left(\lambda_{A_{g}(2)} / N(0)=0.002 \mathrm{eV}\right)$. These calculations do not include the screening effects discussed below. The electronphonon interaction has also been calculated by Jishi and Dresselhaus (1992) and by Chen and Goddard (1993).

Table IV illustrates that there are substantial differences between the different calculations in terms of the distribution of coupling strength between the different modes. Thus Varma et al. (1991) found that almost all the coupling strength is to the two highest modes. Antropov et al. (1993b) found more coupling strength to the lower phonons and this was still more true for the calculations of Schluter et al. (1992b) and Faulhaber et al. (1993). Nevertheless, all four calculations find the strongest coupling to one of the uppermost phonons. The large variation in the distribution of coupling between the different phonons in Table IV may to a substantial extent be due to the sensitivity to the phonon eigenvectors discussed below Eq. (7). These variations suggest that there are substantial uncertainties in the calculations.

In estimates of the total $\lambda$, the contributions from the $\mathrm{A}_{g}$ modes are usually not included. The reason is that the $\mathrm{A}_{g}$ contribution is expected to be screened out in $\mathrm{A}_{3} \mathrm{C}_{60}(\mathrm{~A}=\mathrm{K}, \mathrm{Rb})$ (Schluter et al., 1992b). This is illustrated by Raman scattering results for $\mathrm{A}_{3} \mathrm{C}_{60}(\mathrm{~A}=\mathrm{K}, \mathrm{Rb})$ (Duclos et al., 1991, Pichler et al., 1992). As discussed in Sec. III.3, the additional broadening of the $\mathrm{A}_{g}$ modes in $\mathrm{A}_{3} \mathrm{C}_{60}$ compared with $\mathrm{C}_{60}$ should be a measure of the electron-phonon coupling. Experiments, however, show little or no extra broadening of the $\mathrm{A}_{g}(2)$ in the metallic phase. For instance, Pichler et al. (1992) estimated the additional broadening to be only about $3 \mathrm{~cm}^{-1}$, implying $\lambda_{A_{g}(2)} / N(0) \sim 0.0002$, i.e., an extremely small coupling.

The corresponding additional broadening for many of the $\mathrm{H}_{g}$ modes is, on the other hand, large, indicating a much stronger coupling for these modes. The symmetric $\mathrm{A}_{g}$ mode leads to the same displacement of all the $t_{1 u}$ levels on a given molecule, and this displacement can largely be compensated by a transfer of charge to this molecule. This screening is much less efficient for the $\mathrm{H}_{g}$ modes, since these do not shift the center of gravity of the $t_{1 u}$ levels. Simple estimates within RPA and assuming static screening, suggest that the screening could reduce the coupling to the $\mathrm{A}_{g}$ mode by one to two orders of magnitude (Antropov et al., 1993b). It would be interesting to investigate nonadiabatic effects, going beyond static screening, since the $\mathrm{A}_{g}(2)$ phonon energy is comparable to the energy scale of the screening.
TABLE V. The density of states $N(0)$ (per eV and spin) for $\mathrm{K}_{3} \mathrm{C}_{60}$ and $\mathrm{Rb}_{3} \mathrm{C}_{60}$ according to different band structure calculations. Some of the results have been interpolated by Gelfand (1994).

\begin{tabular}{cc}
\hline \hline$N(0)\left(\mathrm{K}_{3} \mathrm{C}_{60}\right)$ & $N(0)\left(\mathrm{Rb}_{3} \mathrm{C}_{60}\right) / N(0)\left(\mathrm{K}_{3} \mathrm{C}_{60}\right)$ \\
\hline $6.6^{\mathrm{a}}$ & $1.26^{\mathrm{a}}$ \\
$7.0^{\mathrm{b}}$ & \\
$8.6^{\mathrm{c}}$ & $1.18^{\mathrm{c}}$ \\
$8.6^{\mathrm{d}}$ & $1.21^{\mathrm{d}}$ \\
$9.0^{\mathrm{e}}$ & $1.14^{\mathrm{e}}$ \\
$9.8^{\mathrm{f}}$ & $1.27^{\mathrm{f}}$ \\
\hline \hline
\end{tabular}

${ }^{\mathrm{a}}$ Erwin and Pickett $(1991 ; 1992)$

${ }^{\mathrm{b}}$ Antropov (1992)

${ }^{\mathrm{c}}$ Huang et al. (1992)

${ }^{\mathrm{d}}$ Satpathy et al. (1992)

${ }^{\mathrm{e}}$ Novikov et al. (1992)

${ }^{\mathrm{f}}$ Troullier and Martins (1992)

To obtain values of $\lambda$, we also need estimates of the density of states at the Fermi energy $N(0)$. There have been several theoretical calculations of $N(0)$ for $\mathrm{K}_{3} \mathrm{C}_{60}$. Full potential, linear combination of Gaussian orbitals gave $N(0)=6.6$ states $/ \mathrm{eV}$-spin for the socalled uni-directional structure, where all the $\mathrm{C}_{60}$ molecules have the same orientation and there is one $\mathrm{C}_{60}$ molecule per unit cell (Erwin and Pickett, 1991; 1992). An ASA-LMTO calculation gave $N(0)=8.6$ states $/ \mathrm{eV}$-spin (Satpathy et al., 1992 ) and a full potential LMTO gave 7.0 state/eV-spin (Antropov, 1992). These and some other results are collected in Table V. A calculation for the bi-directional structure, where the molecules have two different directions, gave $N(0)=7.5$ states/eV-spin (Antropov, 1992). The most relevant calculation is probably, however, for the orientationally disordered structure. This has only been performed within a tight-binding approach (Gelfand and $\mathrm{Lu}, 1992 \mathrm{ab} ; 1993)$. All the structures in $N(\varepsilon)$ are then smeared out, and $N(0)$ is slightly larger than $3 / W$, where $W$ is the band width. If we assume $W \sim 0.6$ $\mathrm{eV}$, as many band structure calculations find (Erwin and Pickett, 1991; 1992; Antropov, 1992), this gives N(0) of the order 5-6 states/eV-spin.

Experimentally, the density of states has been estimated using a number of different techniques. Many estimates are based on the NMR relaxation rate. It has often been assumed that the Fermi contact interaction dominates the relaxation, and large values of $N(0)$ were then obtained. It has, however, been shown that the relaxation is dominated by the spin-dipolar interaction, due to the $p_{z}$ character $(\sim 95 \%)$ of the states close to the Fermi energy (Antropov et al., 1993a). These results were supported by Tycko et al. (1993), who found that the spin-dipolar coupling dominates for $\mathrm{RbC}_{60}$ and $\mathrm{CsC}_{60}$. Antropov et al. (1993) deduced that $N(0)=7.2$ and 8.1 states/eV-spin for $\mathrm{K}_{3} \mathrm{C}_{60}$ and $\mathrm{Rb}_{3} \mathrm{C}_{60}$, respec- 
tively. These values are used in the following.

$N(0)$ has also been estimated from the specific heat jump $\Delta C$ at the superconductivity transition (Ramirez et al., 1992a; Burkhart and Meingast, 1996). Using the formula

$$
\frac{\Delta C}{T_{c}} \sim N(0)(1+\lambda)\left(1.43+O\left(\lambda^{2}\right)\right),
$$

and $\lambda=0.068 N(0)$ (Antropov et al., 1993b) Burkhart and Meingast (1996) obtained $N(0)=6.6$ and 7.4 states/eV-spin for $\mathrm{K}_{3} \mathrm{C}_{60}$ and $\mathrm{Rb}_{3} \mathrm{C}_{60}$, respectively. Using instead $\lambda=0.147 N(0)$ (Gunnarsson et al., 1995), they found $N(0)=5.3$ and 5.9 states $/ \mathrm{eV}$-spin for $\mathrm{K}_{3} \mathrm{C}_{60}$ and $\mathrm{Rb}_{3} \mathrm{C}_{60}$, respectively.

The density of states has furthermore been estimated from Pauli contribution to the susceptibility, obtained from SQUID and EPR measurements, as in Table VI. The SQUID results were corrected for the diamagnetic contribution from the cores. The result of Wong et al. (1992) was also corrected for the Landau orbital diamagnetism, while Ramirez et al. (1992b) argued that this contribution is negligible in the present systems. The EPR measurements contain no diamagnetic contributions. The susceptibility may have an appreciable Stoner enhancement (Ramirez et al., 1992b). Based on LDA calculations, this enhancement factor was estimated to be 1.34 and 1.42 for $\mathrm{K}_{3} \mathrm{C}_{60}$ and $\mathrm{Rb}_{3} \mathrm{C}_{60}$, respectively (Antropov et al., 1993a). The accuracy of the LDA in this context is, however, not known. This Stoner enhancement has not been taken into account in Table VI, and the numbers in the table should be divided by the appropriate Stoner enhancement. The susceptibility has recently been calculated for a three-fold degenerate Hubbard model, describing the $t_{1 u}$ band and the on-site Coulomb interaction $U$. A quantum Monte Carlo lattice method was used to obtain the enhancement of the Pauli susceptibility due to the Coulomb interaction (Aryasetiawan et al., 1996). For typical values of $U \sim 1.2-1.3 \mathrm{eV}$, an enhancement of the order of a factor three was obtain. This would reduce the values of $N(0)$ estimated from the susceptibility and roughly bring them in line with other estimates.

We observe that it is hard to estimate $N(0)$ from photoemission or yield spectroscopy (De Seta and Evangelisti, 1995), due to a large broadening of the band, which is probably caused by phonon and plasmon satellites (Knupfer et al., 1993) or other many-body effects. Various experimental estimates of $N(0)$ have been discussed by Gelfand (1994).

Table VII shows the contribution from the $\mathrm{H}_{g}$ modes to $\lambda$ using the values of $N(0)$ deduced from NMR, i.e., $N(0)=7.2$ and 8.1 states $/ \mathrm{eV}$-spin for $\mathrm{K}_{3} \mathrm{C}_{60}$ and $\mathrm{Rb}_{3} \mathrm{C}_{60}$, respectively.
TABLE VI. The density of states $N(0)$ (per eV and spin) for $\mathrm{K}_{3} \mathrm{C}_{60}$ and $\mathrm{Rb}_{3} \mathrm{C}_{60}$ as deduced from susceptibility measurements. The results have not been corrected for the Stoner enhancement, which would lead to reduced estimates of $N(0)$.

\begin{tabular}{ccc}
\hline \hline$N(0)\left(\mathrm{K}_{3} \mathrm{C}_{60}\right)$ & $N(0)\left(\mathrm{Rb}_{3} \mathrm{C}_{60}\right) / N(0)\left(\mathrm{K}_{3} \mathrm{C}_{60}\right)$ & Method \\
\hline $14^{\mathrm{a}}$ & $1.36^{\mathrm{a}}$ & SQUID \\
$16^{\mathrm{b}}$ & & SQUID \\
$11^{\mathrm{b}}$ & & EPR \\
$15^{\mathrm{c}}$ & $1.40^{\mathrm{d}}$ & EPR \\
$10^{\mathrm{d}}$ & & EPR \\
\hline \hline
\end{tabular}

${ }^{\mathrm{a}}$ Ramirez et al. (1992b)

${ }^{\mathrm{b}}$ Wong et al. (1992)

${ }^{\mathrm{c}}$ Tanigaki et al. (1995)

${ }^{\mathrm{d}}$ Wang et al. (1995)

TABLE VII. The $\mathrm{H}_{g}$ contribution to $\lambda$ according to different theoretical calculations, using $N(0)=7.2$ and 8.1 states per $\mathrm{eV}$ and spin for $\mathrm{K}_{3} \mathrm{C}_{60}$ and $\mathrm{Rb}_{3} \mathrm{C}_{60}$, respectively.

\begin{tabular}{ccccc}
\hline \hline Mode & & \multicolumn{3}{c}{$\lambda_{\nu}$} \\
& Varma $^{\mathrm{a}}$ & Schluter $^{\mathrm{b}}$ & Antropov $^{\mathrm{c}}$ & Faulhaber $^{\mathrm{d}}$ \\
\hline $\mathrm{K}_{3} \mathrm{C}_{60}$ & 0.40 & 0.37 & 0.49 & 0.35 \\
$\mathrm{Rb}_{3} \mathrm{C}_{60}$ & 0.45 & 0.42 & 0.55 & 0.40 \\
\hline \hline
\end{tabular}

${ }^{\text {a }}$ Varma et al., 1991

${ }^{\mathrm{b}}$ Schluter et al., 1992

${ }^{\mathrm{c}}$ Antropov et al., 1993

${ }^{\mathrm{d}}$ Faulhaber et al., 1993

It is interesting to compare the alkali-fullerides with intercalated graphite, since the latter have much lower values (one to two orders of magnitude) of $T_{c}$ than the fullerides. It has been reported that calculations for intercalated graphite gives a substantially lower value (factor five) of $\lambda$ than for the fullerides (Schluter et al., 1992a). This is not ascribed to a larger density of states in the fullerides but to the curvature of the $\mathrm{C}_{60}$ molecule (Benning et al., 1991; Schluter et al., 1992ab). In intercalated graphite, to first order there is no coupling to displacements perpendicular to the graphite planes. In $\mathrm{C}_{60}$, on the other hand, the curvature mixes in $2 s$ character in the primarily radial $2 p$ orbital pointing out from the molecule and dominating the states in the $t_{1 u}$ band. This allows for a coupling of these states to radial (transverse) modes. Alternatively, we can think of the displacements of the nuclei as setting up an approximately $p$-like perturbing potential close to the nuclei. Such a potential can couple a $2 s$ and a $2 p$ orbital but not two $2 p$ orbitals on the same site. Pietronero and Strässler (1992) suggested as an alternative explanation that the violation of Migdal's theorem in $\mathrm{A}_{3} \mathrm{C}_{60}$ may cause the large $T_{c}$ in these compounds. 


\section{Raman and neutron scattering}

Due to the electron-phonon coupling, a phonon can decay in an electron-hole pair of the appropriate energy. In the metallic fullerides $\mathrm{A}_{3} \mathrm{C}_{60}$ this decay channel is available and contributes to the width of the phonons. This width is therefore a measure of the electron-phonon coupling (Allen, 1972; Allen, 1974) and for the $\nu$ th phonon it is given by

$$
\gamma_{\nu}=\frac{2 \pi \omega_{\nu}^{2} N(0) \lambda_{\nu}}{g_{\nu}}
$$

where $\gamma_{\nu}$ is the full width at half maximum (FWHM), $\omega_{\nu}$ is the phonon frequency, $N(0)$ is the density of states per spin, $\lambda_{\nu}$ the electron-phonon coupling and $g_{\nu}$ is the degeneracy of the phonon ( 5 for $\mathrm{H}_{g}$ ). Due to the factor $\omega_{\nu}^{2}$, the width of the high-lying phonons is often very large and those phonons are hard to observe experimentally.

The width can be measured using Raman or neutron scattering. In an ordered system, Raman scattering samples the decay of a phonon for $\mathbf{q} \approx 0$ and it may be misleading for the decay in the rest of the Brillouin zone. In $\mathrm{A}_{3} \mathrm{C}_{60}(\mathrm{~A}=\mathrm{K}, \mathrm{Rb})$ there is, however, a strong orientational disorder (Stephens et al., 1991) and Raman scattering should be relevant for the determination of the electron-phonon coupling integrated over q (Schluter, 1992c). The observed widths may therefore include some dispersion effects. These are, however, expected to be small for the intramolecular modes, due to the weak coupling between the molecules (Belosludov and Shpakov, 1992).

The widths of some of the $\mathrm{H}_{g}$ phonons were measured very early by Mitch et al. (1992a, 1992b). They performed Raman measurements for ultrathin films of alkalidoped $\mathrm{A}_{x} \mathrm{C}_{60}$ compounds. Due to the small thickness of the film, they were able to continuously dope the system with $\mathrm{Na}, \mathrm{K}, \mathrm{Rb}$, Cs. For the $\mathrm{H}_{g}(2)$ mode they observed a half-width of $14,22,22$ and $20 \mathrm{~cm}^{-1}$ for $\mathrm{A}=\mathrm{Na} x=1$, $\mathrm{A}=\mathrm{K} x=2.1, \mathrm{~A}=\mathrm{Rb} x=1.9$ and $\mathrm{A}=\mathrm{Cs} x=2$, respectively. They assigned the rather $x$-independent broadening in $\mathrm{NaC}_{60}$ to disorder, and assumed that the disorder is similar in the other compounds. The additional broadening in these compounds was assigned to the electronphonon coupling. Assuming that the two mechanisms add quadratically, they deduced the widths $17 \mathrm{~cm}^{-1}$ for $\mathrm{K}$ and $\mathrm{Rb}$ doped $\mathrm{C}_{60}$. To obtain a value for $\lambda_{2}$ from Eq. (9), one further has to assume a value for $N(0)$. Since different groups have used different values for $N(0)$, we have here uniformly used $N(0)=7.2 \mathrm{eV}^{-1}$ per spin, deduced from NMR for $\mathrm{K}_{3} \mathrm{C}_{60}$ (Antropov et al., 1993a), to be able to compare the different estimates. The values quoted below therefore differs from the values of $\lambda$ given in the original papers. In a few cases below we have also corrected for an incorrect prefactor in Eq. (9) which has appeared in the literature. In Table VIII we show different experimental estimates of $\lambda_{\nu} / N(0)$ for $\mathrm{K}_{3} \mathrm{C}_{60}$.

The phonon width has also been studied in neutron scattering for $\mathrm{C}_{60}, \mathrm{~K}_{3} \mathrm{C}_{60}$ and $\mathrm{Rb}_{6} \mathrm{C}_{60}$ by Prassides et al. (1992). By comparing the broadening for the metallic $\mathrm{K}_{3} \mathrm{C}_{60}$ and the insulating $\mathrm{C}_{60}$, the additional broadening due to the electron-phonon coupling was deduced. This was performed for the four lowest $\mathrm{H}_{g}$ modes. The result for $\mathrm{H}_{g}(2)$ is in good agreement with the result of Mitch et al. (1992b).

Recently, the Raman spectrum was studied by Winter and Kuzmany (1996) for single crystals and at low temperatures. They found that the peaks for several of the $\mathrm{H}_{g}$ phonons were strongly asymmetric and could be fitted with five components. This is shown in Fig. 7.

TABLE VIII. The partial electron-phonon coupling constants $\lambda_{\nu} / N(0)$ [in $\mathrm{eV}$ ] according to different experimental estimates. We also show the energies $\left(\right.$ in $\mathrm{cm}^{-1}$ ) of the modes for the undoped system.

\begin{tabular}{lccccc}
\hline \hline Mode & Energy & \multicolumn{4}{c}{$\lambda_{\nu} / N(0)$} \\
& & Raman $^{\mathrm{a}}$ & Neutron $^{\mathrm{b}}$ & Raman $^{\mathrm{c}}$ & PES $^{\mathrm{d}}$ \\
\hline $\mathrm{H}_{g}(8)$ & 1575 & & & .002 & .023 \\
$\mathrm{H}_{g}(7)$ & 1428 & & & .003 & .017 \\
$\mathrm{H}_{g}(6)$ & 1250 & & & .001 & .005 \\
$\mathrm{H}_{g}(5)$ & 1099 & & & .001 & .012 \\
$\mathrm{H}_{g}(4)$ & 774 & & .005 & .002 & .018 \\
$\mathrm{H}_{g}(3)$ & 710 & & .001 & .002 & .013 \\
$\mathrm{H}_{g}(2)$ & 437 & .022 & .023 & .014 & .040 \\
$\mathrm{H}_{g}(1)$ & 273 & & .014 & .034 & .019 \\
\hline \hline
\end{tabular}

${ }^{\mathrm{a}}$ Mitch et al., $1992 \mathrm{~b}$

${ }^{\mathrm{b}}$ Prassides et al., 1992

${ }^{\mathrm{c}}$ Winter et al., 1995

${ }^{\mathrm{d}}$ Gunnarsson et al., 1995

It is interesting that the component with the largest shift relative to the position in the undoped crystal also has the largest width. This suggests that the different components have different electron-phonon couplings. The origin of such a difference in the coupling, if it exists, is however not clear. It is also interesting that the two highest $\mathrm{H}_{g}$ modes seemed to have a different pattern of splitting. The observed line shape therefore poses a very interesting theoretical problem. If it is assumed that the formula of Allen $(1972,1974)$ is still applicable to each component by itself, the results in Table VIII are obtained.

The measurement of the phonon broadening provides rather direct information about the electron-phonon coupling. We note, however, that the interpretation (Allen, 1972) is based on Migdal's theorem. Although this should be an excellent approximation for most systems, it is questionable for the fullerides since the phonon frequencies and the $t_{1 u}$ band width are comparable (Pietronero and Strässler, 1992; 1994; Pietronero et al., 1995; Grimaldi et al., 1995ab). There are also questions 
if Jahn-Teller like effects studied for free molecules (Auerbach et al., 1994; Manini et al., 1994) and going beyond Migdal's theorem, could survive in the solid and influence the results. Such higher order effects are very important for the photoemission spectrum of a free molecule discussed below. In particular, the interesting line shape obtained by Winter and Kuzmany (1996) raises questions about our understanding of how to analyze the spectra.

Reviews of Raman and neutron scattering studies of the fullerides have been given by Kuzmany et al. (1994b) and by Pintschovius (1966), respectively.

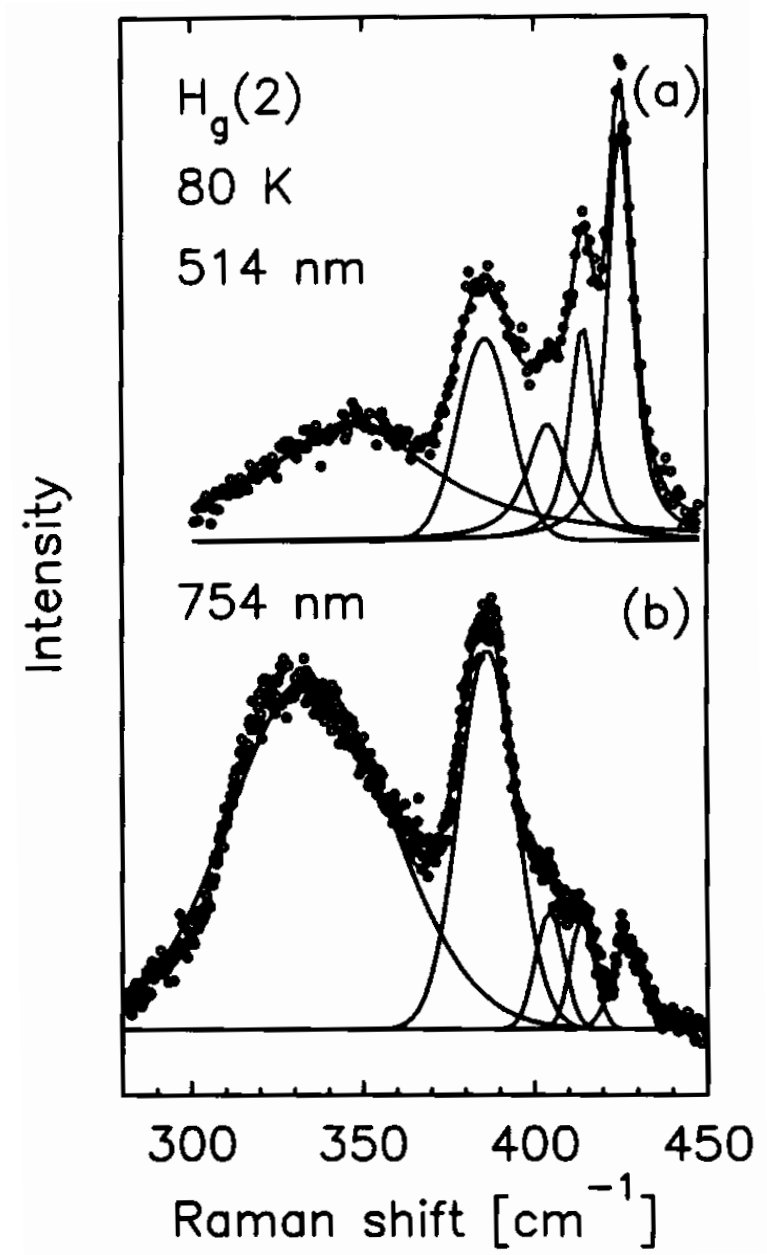

FIG. 7. Raman spectrum of the $\mathrm{H}_{g}(2)$ mode excited with a green laser (a) and with a red laser (b). The full lines show a fit to five Voigtian profiles (after Winter and Kuzmany, 1996).

\section{Photoemission}

An alternative way of deducing the electron-phonon coupling is the use of photoemission data. Because of the relatively strong electron-phonon coupling, we expect to see satellites due to the excitation of phonons. The weight of the satellites should then give information about the strength of the coupling. This is essentially the Franck-Condon effect, except that it is important to take the Jahn-Teller effect into account for $\mathrm{C}_{60}$. The photoemmision spectra of $\mathrm{K}_{3} \mathrm{C}_{60}$ and $\mathrm{Rb}_{3} \mathrm{C}_{60}$ have been analyzed along these lines (Knupfer et al., 1993). Due to the broadening effects in a solid and due to the complications in the theoretical treatment of bands with dispersion, it was not possible, however, to derive reliable, quantitative values for the electron-phonon coupling.

Photoemission experiments have, however, also been performed for free $\mathrm{C}_{60}^{-}$molecules, where the theoretical treatment is substantially simpler (Gunnarsson et al., 1995). In these experiments a beam of $\mathrm{C}_{60}^{-}$ions was created and a photoemission experiment was performed using a laser light source $(\hbar \omega=4.025 \mathrm{eV})$ and a time of flight spectrometer. To analyse the results, a model was used which includes the $t_{1 u}$ level from which the electron is emitted, the two $\mathrm{A}_{g}$ and eight, five-fold degenerate $\mathrm{H}_{g}$ modes and their coupling to the electronic state. For this model the ground-state can be calculated by numerical diagonalization to any desired accuracy. Within the sudden approximation (Hedin and Lundqvist, 1969), the photoemission spectrum can furthermore easily be calculated. A set of coupling constants are then assumed and the resulting spectrum is compared with experiment. The coupling parameters are then varied until good agreement with experiment is obtained, thereby providing an estimate of the couplings. The resulting spectrum is compared with experiment in Fig. 8 and the corresponding parameters are shown in Table VIII.

This approach contains mainly three uncertainties. Firstly, it is based on the sudden approximation. This approximation should become very accurate as the kinetic energy of the emitted electron becomes large. Due to the small photon energy used, to obtain a good resolution, the accuracy of the sudden approximation is unclear. Spectra taken at different photon energies did not, however, show any systematic variation, suggesting that the sudden approximation may not be a serious problem here. We further observe that the weight of the satellites must go to zero as the photon energy is reduced sufficiently. If this happens in a monotonous way, the use of the sudden approximation would underestimate the couplings. As shown in Table VIII, the couplings derived from photoemission, however, tend to be larger than other estimates. The second uncertainty is more technical, and is related to the fact that for the resolution available, it is not possible to distinguish between the coupling to the $\mathrm{A}_{g}$ modes and the $\mathrm{H}_{g}$ modes with similar energies. The couplings to the $\mathrm{A}_{g}$ modes were therefore taken from a calculation (Antropov et al., 1993b). With this assumption the couplings to the $\mathrm{H}_{g}$ modes can then be determined uniquely. An equally good fit to experiment could, however, be obtained if, for instance, the coupling to the $\mathrm{A}_{g}(2), \lambda_{A_{g}(2)} / N(0)$, was allowed to vary 
between 0.00 and $0.03 \mathrm{eV}$, provided that the total coupling to the $\mathrm{H}_{g}(7)$ and $\mathrm{H}_{g}(8)$ modes were simultaneously varied between 0.07 and $0.00 \mathrm{eV}$. Thirdly, the coupling is determined for $\mathrm{C}_{60}^{-}$, while we are interested in $\mathrm{C}_{60}^{3-}$ in a solid. Although the additional charging of $\mathrm{C}_{60}$ is not expected to have large effects, due to the sensitivity of the coupling to the phonon eigenvectors, the coupling could still be influenced.

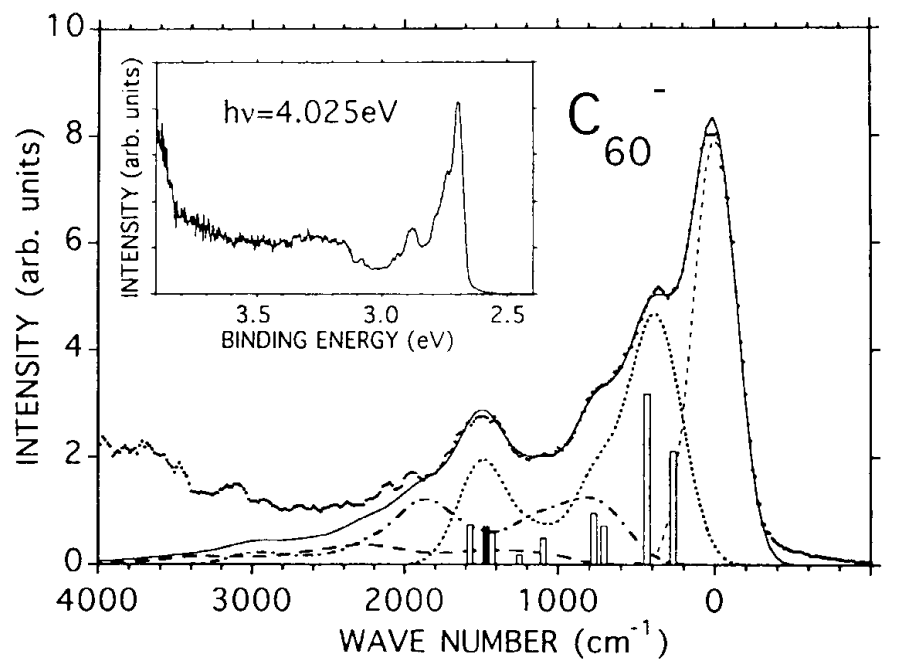

FIG. 8. The experimental (dots) and theoretical (full line) photoemission spectrum of $\mathrm{C}_{60}^{-}$. The theoretical no loss (dashed), single loss (dotted) and double loss (dasheddotted) curves are also shown. The contributions of the different modes to the single loss curve are given by bars $\left(\mathrm{H}_{g}\right.$ : open, $\mathrm{A}_{g}$ : solid). The inset shows the experimental spectrum over a larger energy range (After Gunnarsson et al., 1995).

\section{Resistivity}

The electric resistivity can be caused by various scattering mechanisms. An important mechanism is often the electron-phonon interaction, where electrons are scattered under the simultaneous excitation of phonons. Another mechanism is provided by the electron-electron scattering. The contribution from both these mechanisms goes to zero as $T \rightarrow 0$. Deviations from periodicity, e.g., the orientational disorder, on the other hand, leads to an essentially temperature independent resistivity. If the dominating contribution to the $T$-dependent part of the resistivity comes from the electron-phonon scattering, the resistivity provides an interesting check of the values of the electron-phonon coupling constants.

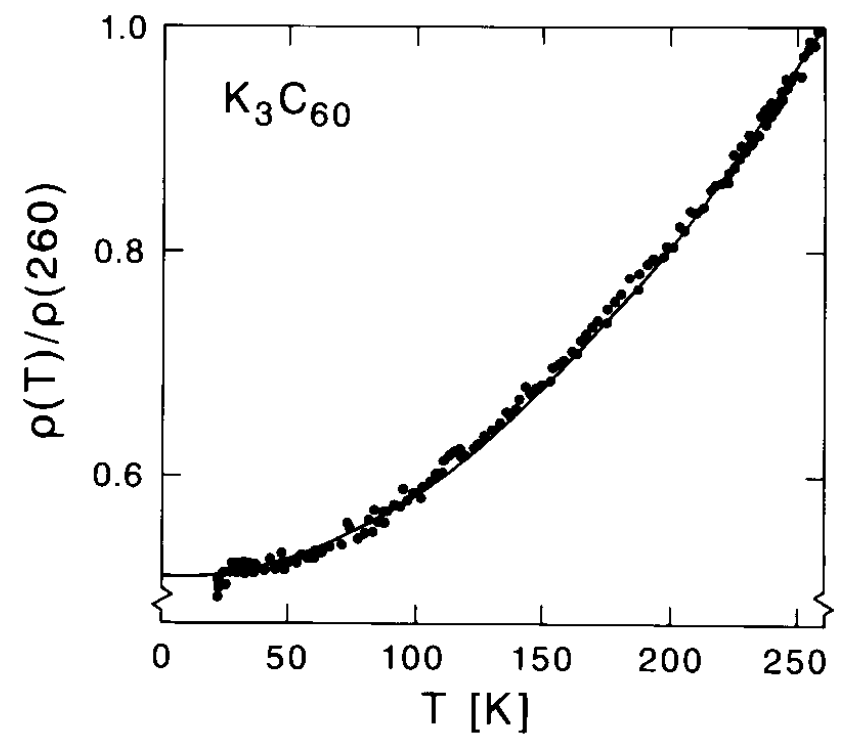

FIG. 9. The experimental (dots)(Xiang et al., 1992) and theoretica l (full curve) $T$-dependent resistivity $\rho(T)$ normalized to the value at $T=260 \mathrm{~K}$.

In Fig. 9 typical results for the temperature dependence are shown. For low $T$, it is found that the temperature-dependent part of $\rho(T)$ at constant pressure is approximately quadratic in $T$ (Crespi, 1992). This suggests that the mechanism at these values of $T$ is electronelectron scattering (Palstra et al., 1994). To obtain a reasonable magnitude, it is, however, necessary to assume that the matrix element for electron-electron scattering is of the order $1 \mathrm{eV}$, i.e., comparable to the $t_{1 u}$ band width. Such a large matrix element would suggest a rather inefficient screening. The approximate $T^{2}$ behavior can, however, also be obtained from the electron-phonon mechanism with a reasonable distributions of coupling constants. Therefore we focus on this mechanism below. More recent experimental work (Vareka and Zettl, 1994) has suggested that $\rho(T)$ at constant volume is linear in $T$ down to relatively low $T(100-200 \mathrm{~K})$. This raises new questions, and the resistivity can not be considered as fully understood.

The resistivity has been studied theoretically by several groups (Crespi et al., 1992; Erwin and Pickett, 1992; Antropov et al., 1993b; Lannin and Mitch, 1994), using the theory of Ziman (Grimvall, 1981). This theory provides an approximate solution to the Boltzmann equation for the case of the electron-phonon scattering mechanism.

$$
\begin{aligned}
& \rho(T)=\frac{8 \pi^{2}}{\omega_{p}^{2} k_{B} T} \\
& \times \int_{0}^{\omega_{\max }} d \omega \frac{\hbar \omega \alpha_{t r}^{2} F(\omega)}{\cosh \left(\hbar \omega / k_{B} T\right)-1}
\end{aligned}
$$

where $\omega_{p}$ is the plasma frequency. In the following it is assumed that the transport coupling function $\alpha_{t r}^{2} F(\omega)$ 
can be replaced by the electron phonon coupling appropriate for superconductivity

$$
\alpha^{2} F(\omega)=\frac{1}{2} \sum_{\nu} \omega_{\nu} \lambda_{\nu} \delta\left(\omega-\omega_{\nu}\right)
$$

Due to the factor $\cosh \left(\hbar \omega / k_{B} T\right)-1$ in the denominator of Eq. (10), it is clear that phonons with a frequency much larger than $T$ do not contribute, while they give a linear contribution in $T$ if their frequency is much smaller than $T$. Using this approach, Crespi et al. (1992) demonstrated that theoretical results for the coupling constants to the $\mathrm{H}_{g}$ modes alone of the type obtained by Varma et al. (1991) or Schluter et al. (1992) gave a poor fit of experiment, giving a much too weak temperature dependence at small $T$. The reason is that the $\mathrm{H}_{g}$ phonons are too high in energy to give much contribution to $\rho(T)$ at low $T$, in particular since in these theories most of the coupling occurs to the higher phonons. The couplings of Jishi and Dresselhaus (1992), emphasizing the lower $\mathrm{H}_{g}$ phonons, gave better agreement. Crespi et al. (1992) argued that one also has to consider low-lying external modes, e.g., the intermolecular translational modes. Including such modes, they showed that the couplings of Varma et al. (1991), Schluter et al. (1992) and Jishi and Dresselhaus (1992) give reasonable descriptions of the experimental $T$-dependence, if the coupling to the translational mode is chosen appropriately.

As an example of such results, we show a calculation using the coupling constants deduced from photoemission (Gunnarsson, 1995). In addition, the calculation included the coupling to the librations, using a theoretical coupling constant (Antropov et al., 1993b), and to the intermolecular modes, treating the coupling as an adjustable parameter, since its value is unknown. As in the calculation of Crespi et al. (1992), the energy of the intermolecular mode was set to $110 \mathrm{~cm}^{-1}$, since neutron scattering shows many modes at this energy. We have set $\omega_{p}=1.36 \mathrm{eV}$. It was further assumed that some other mechanism, e.g., orientational disorder, provides a $T$-independent resistivity which accounts for the resistivity just above $T_{c}$. The results are shown in Fig. 9 . We can see that there is a good agreement with experiment over the whole temperature range. In particular, the approximate $T^{2}$ behavior is reproduced. This results from the contribution of many phonons at different energies, whose contributions to the resistivity are gradually switched on one after the other as $T$ is increased. For high temperatures in Fig. 9, the resistivity corresponds to a mean free path of the order of or less than the separations between the molecules. This raises serious questions about the validity of the Boltzmann equation for these temperatures.

The theoretical and experimental results in Fig. 9 are for constant pressure. The experimental data have also been converted to constant volume, by measuring the pressure dependence (Vareka and Zettl, 1994). The resistivity then shows an approximately linear behavior for temperatures larger than about 100 to $200 \mathrm{~K}$. This linear behavior appears not to have been explained theoretically, and it is an interesting problem.

It is of particular interest to consider the absolute value of the resistivity increase with $T$, e.g., $\Delta \rho \equiv \operatorname{rho}(T=$ $260)-\rho(T=0)$, since that may give information about the magnitude of $\lambda$. Many estimates of the resistivity, however, are made for low temperatures, thereby giving the residual resistivity and not $\Delta r h o$. Since the shape of $\rho(T)$, but not its absolute magnitude, appears to be well reproducible (Xiang et al., 1992), we use the experimental ratio $\Delta \rho / \rho(T=0)$ two relate etimates of $\Delta$ rho and $\rho(T=0)$. Here $\rho(T=0)$ refers to the resistivity just above the superconductivity transition temperature. Thus we convert our theoretical estimate of $\Delta r h o$ to a theoretical estimate of $\rho(T=0)=0.47 \mathrm{~m} \Omega \mathrm{cm}$. It is interesting that the resistivity from orientational disorder, $0.32 \mathrm{~m} \Omega \mathrm{cm}$ (Gelfand and Lu, 1992b; 1993), is not much smaller. Direct measurements for thin films and single crystals gave $\rho(T=0) \sim 1.2 \mathrm{~m} \Omega \mathrm{cm}$ (Palstra et al., 1994) and $\rho(T=0)=0.5 \mathrm{~m} \Omega \mathrm{cm}$ (Hou et al., 1993). Since the direct measurements involve various uncertainties (possibly somewhat granular structure for the thin films and geometrically very small samples for the single crystals), it is also interesting to compare with indirect measurements of the resistivity. Xiang et al. (1993) deduced $\rho(T=0)=0.12 \mathrm{~m} \Omega \mathrm{cm}$ from the paraconductivity (additional conductivity due to superconducting fluctuations just above $T_{c}$ ) and Crespi et al. (1992) deduced $\rho(T=0)=0.18 \mathrm{~m} \Omega \mathrm{cm}$ from the upper critical field. Degiorgi et al. (1992) obtained $\rho(T=0)=0.77$ $\mathrm{m} \Omega \mathrm{cm}$ from a Kramer-Kronig analysis of the reflectivity in the normal state and Rotter et al. (1992) found $\rho(T=0)=0.4 \mathrm{~m} \Omega \mathrm{cm}$ from the ratio between the reflectivity in the normal and superconducting state. These results are summarized in Table IX.

Comparison between the theoretical and experimental estimates shows that the theoretical estimate falls in the correct range. Given the large range of experimental estimates it is, however, hard to assess the accuracy of the theoretical calculation. We also observe that the theoretical result was obtained from a set of coupling parameters which give very strong couplings to the low-lying modes, which leads to a large resistivity, while several other estimates of the couplings would give a substantially smaller resistivity.

The residual resistivity is smaller than the Mott limit, which, due to the small conduction electron density, is rather large for these systems, and has been estimated to be 1.4-2.0 $\mathrm{m} \Omega \mathrm{cm}$ (Palstra et al., 1994). For high temperatures, the resistivity corresponds to a mean free path shorter than the separation between the molecules. In such a situation the resistivity is usually expected to sat- 
urate at a maximum value (Fisk and Webb, 1976). Such a saturation is not observed for the doped $\mathrm{C}_{60}$ compounds up to rather high temperatures $(T \lesssim 500 \mathrm{~K}$ ) (Hebard et al., 1993), but for still larger temperatures it is seen for $\mathrm{Rb}_{3} \mathrm{C}_{60}$ (Hou et al, 1995). The saturation value was estimated to $\rho_{\text {sat }}=6 \pm 3 \mathrm{~m} \Omega$-cm with the corresponding mean free path $l_{\text {sat }}=1 \pm 0.5 \AA$, significantly smaller than the separation between the molecules. The reason for this small mean free path appears not to be understood, and it is an interesting question how one should conceptionally understand such a short mean free path.

TABLE IX. The resistivity $\rho(0)$ in $\mathrm{m} \Omega \mathrm{cm}$ just above the superconductivity transition according to different theoretical and experimental estimates. For details see text.

\begin{tabular}{ll}
\hline \hline$\rho(0)$ & Method \\
\hline 0.47 & Theory. $\rho(260)-\rho(0) \rightarrow \rho(0)$ \\
$0.32^{\mathrm{a}}$ & Theory. Disorder \\
$1.2^{\mathrm{b}}$ & Direct. Film \\
$0.5^{\mathrm{c}}$ & Direct. Single crystal \\
$0.12^{\mathrm{d}}$ & Paraconductivity \\
$0.18^{\mathrm{e}}$ & Upper critical field \\
$0.77^{\mathrm{f}}$ & Reflectivity. K-K \\
$0.4^{\mathrm{g}}$ & Reflectivity. S-N \\
\hline \hline
\end{tabular}

${ }^{\mathrm{a}}$ Gelfand and Lu (1992b, 1993)

${ }^{\mathrm{b}}$ Palstra et al. (1994)

${ }^{\mathrm{c}} \mathrm{Hou}$ et al. (1993)

${ }^{\mathrm{d}}$ Xiang et al. (1993)

${ }^{\mathrm{e}}$ Crespi et al. (1992)

${ }^{\mathrm{f}}$ Degiorgi et al. (1992)

${ }^{\text {g}}$ Rotter et al. (1992)

\section{Comparison of electron-phonon coupling estimates}

We are now in the position of comparing different theoretical (Table IV) and experimental (Table VIII) estimates of the coupling constants. We first of all observe that the quantity $\lambda / N(0)$ is obtained in the calculations and is deduced from photoemission, while Raman and neutron scattering gives an estimate of $\lambda N(0)$. Our assumptions about $N(0)$ ( 7.2 states $/ \mathrm{eV}$ per spin for $\left.\mathrm{K}_{3} \mathrm{C}_{60}\right)$ is therefore crucial for these comparisons.

There are substantial differences between the different calculations of $\lambda$, between the different experimental estimates, and between theoretical and experimental values. Experimentally, a strong coupling to some of the low-lying modes is deduced. For instance, the total coupling to the lowest two modes is experimentally several times larger than theoretically. Thus the experimental estimates are 0.037, 0.048 and $0.059 \mathrm{eV}$, according to neutron, Raman and photoemission experiments, respectively, while the theoretical estimates are 0.004, 0.015, 0.009 and $0.011 \mathrm{eV}$. For the high-lying phonons, the calculations give a rather strong coupling in agreement with the results from photoemission but in contradiction with the results from Raman. It is not clear why there is such a large discrepancy between the photoemission and Raman results for these modes.

If a larger value for $N(0)$ is assumed, the discrepancy between the theoretical values and the Raman and neutron estimates for the low-lying modes would be reduced, but the discrepancy with the photoemission values would remain. At the same time the difference between the theoretical and the Raman estimates would be increased for the high-lying modes. As discussed above, relatively small errors in the phonon eigenvectors could lead to a large underestimate of the theoretical coupling to the low-energy modes.

\section{B. Alkali phonons}

Very early it was pointed out that the alkali modes may provide a strong electron-phonon coupling (Zhang et al., 1991a). Each $\mathrm{C}_{60}$ molecule in $\mathrm{A}_{3} \mathrm{C}_{60}$ is surrounded by 14 alkali atoms (6 octahedral and 8 tetrahedral). The corresponding phonon frequencies are low (0.013 eV (Prassides et al., 1992); 0.014 eV (Renker et al., 1993); $0.13 \mathrm{eV}$ (Mitch and Lannin, 1993)), implying weak force constants. If an electron is transferred to a $\mathrm{C}_{60}$ molecule, one may then expect the surrounding alkali ions to move towards the $\mathrm{C}_{60}$ molecule, giving an efficient screening. It has been estimated that the alkali phonons could contribute an attractive interaction of the order 0.9 eV (Zhang et al., 1991a), which corresponds to $\lambda \sim 2$, i.e., a strong coupling. Later experiments demonstrated that $T_{c}$ is approximately independent of the alkali atom mass (see Sec. IIA and IIC), showing that the alkali modes must contribute very little to $T_{c}$. This was rationalized in terms of an efficient metallic screening (Gunnarsson and Zwicknagl, 1992). If an electron is transferred to a $\mathrm{C}_{60}$ molecule, there is a tendency to screen it by transferring charge from that molecule to more distant molecules. The alkali ions then couple essentially to the net charge change of the $\mathrm{C}_{60}$ molecule. Within the RPA this screening mechanism is very efficient, and when an electron is transferred to a $\mathrm{C}_{60}$ molecule the change of its net charge is only a few hundredths of an electron (Gunnarsson and Zwicknagl, 1992). The coupling to the alkali modes is then reduced by one to two orders of magnitude. Although RPA may greatly overestimate the efficiency of the screening, this nevertheless suggests a large reduction of the coupling.

The alkali phonon frequency for the tetrahedral position has been calculated using the density functional formalism in the LD approximation by Bohnen et al. (1995). They found the energy $0.015 \mathrm{eV}$, in good agreement with experiment. 


\section{Librations}

Early point contact measurements of the superconductivity gap gave a large value of $2 \Delta / T_{c}$ (Zhang et al., 1991b), suggesting strong coupling effects and the coupling to low energy modes. It was therefore suggested that the coupling to the librations may be of importance (Dolgov and Mazin, 1992; Mazin et al., 1993b). There are several factors influencing this coupling. For the intramolecular phonons, the main changes in the matrix elements come from changes in bond lengths and bond angels, i.e., changes of the order of the intramolecular energy scale. For the librations, which involve rigid rotations of the molecules, only the hopping between the molecules is influenced, i.e., changes of the order of the intermolecular energy. The amplitude factor in Eq. (6) also tends to be small for the librations due to the large moment of inertia, $I_{i i}=40 M R^{2}$, where $M$ is the carbon mass and $R$ is the $C_{60}$ radius. On the other hand, the very small libration frequency $(\sim 4 \mathrm{meV}$, (Christides et al., 1992 )) tends to increase both the amplitude factor in Eq. (6) and the $\lambda$ in Eq. (5). It is therefore a priori not easy to estimate the magnitude of the coupling to the librations.

We observe that the simplifications in the calculation of the coupling to the intramolecular phonons (Lannoo et al., 1991) do not apply to the librations and the Brillouin zone sums in Eq. (5) should be performed. This has been performed within a tight-binding formalism, which gave $\lambda_{l} / N(0) \sim 0.01$ (Antropov et al., 1993b). Comparing the splitting of the $t_{1 u}$ band at the $\Gamma$ point in the tightbinding calculation and a ASA-LMTO LDA calculation, suggested that $\lambda_{l}$ should be reduced by about a factor 10, giving $\lambda_{l} / N(0) \sim 0.001$ and $\lambda_{l} \sim 0.01$ (Antropov et al., 1993b). Thus the coupling to the librations is found to be very weak.

The coupling has also been studied experimentally (Christides et al., 1992; Reznik et al., 1994). They studied the librations above and below $T_{c}$. Since the libration energy is smaller than the superconductivity gap $2 \Delta$, the broadening of the libration due to the decay in electronhole pairs should go to zero for small temperatures (Zeyher and Zwicknagl, 1990). Experimentally, however, no change in the width was observed when the temperature was lowered below $T_{c}$. It was therefore concluded that $\lambda_{l}<0.08$, since the change in width should otherwise have been observed (Christides et al., 1992).

\section{Intermolecular modes}

The lattice-dynamics of $\mathrm{K}_{3} \mathrm{C}_{60}$ has been studied by Belosludov and Shpakov (1992). They included the van der Waals interaction between the $\mathrm{C}_{60}$ molecules, the electrostatic interactions between the ions, Born-Mayer type of repulsive interactions to describe the repulsion of the ion cores and a valence force model to describe the covalent interaction between the carbon atoms inside a $\mathrm{C}_{60}$ molecule. The parameters were adjusted to reproduce the experimental intramolecular $T_{1 u}$ vibrations. This lead to acoustic modes which at larger wave vectors are mixed with librational modes at about $3.5 \mathrm{meV}$. At larger energies ( $6 \mathrm{meV}$ and $16 \mathrm{meV}$ ) they found bands of alkali and $\mathrm{C}_{60}$ character. This seems to be consistent with neutron scattering experiments (Prassides et al., 1991; Christides et al., 1992). The translational phonons have also been studied by Zhang et al. (1992) for $\mathrm{K}_{3} \mathrm{C}_{60}$ and by Wang et al. (1991) for $\mathrm{C}_{60}$.

Pickett et al. (1994) have studied the electron-phonon coupling for translational modes. They used a tightbinding parameterization of the hopping integrals. A distortion of the lattice corresponding to the longitudinal zone boundary (110) intermolecular optic mode was introduced. From the displacement of the band structure energies they estimated that $\lambda \sim 0.01$ for these modes. The intermolecular modes should therefore have a small effect on $T_{c}$.

\section{COULOMB INTERACTION AND METALLICITY}

In this section we discuss the Coulomb interaction between two electrons on a free $\mathrm{C}_{60}$ molecule $\left(U_{0}\right)$ and for a molecule in the solid $(U)$. The large Coulomb interaction has played an important role in the discussion about superconductivity in the fullerides. This has ranged from the issue of why $\mathrm{A}_{3} \mathrm{C}_{60}$ are not Mott-Hubbard insulators (Lof et al., 1992), over questions about whether or not retardation effects can reduce the Coulomb interaction enough to allow for superconductivity driven by the electron-phonon interaction (Anderson, 1991; Gunnarsson and Zwicknagl, 1992) to the suggestion that the Coulomb interaction could actually be the cause of superconductivity (Chakravarty et al., 1991; Baskaran and Tosatti, 1991; Friedberg et al., 1992).

\section{A. Coulomb $U_{0}$ for a free molecule}

A simple estimate of $U_{0}$ for a free molecule can be obtained by assuming that the charge from an electron is uniformly spread out over a sheet with the radius $R$. The interaction between two electrons is then $e^{2} / R=4.1 \mathrm{eV}$, if $R=3.5 \AA$. This estimate neglects the relaxation of the charge density when an electron is added to the molecule. To include this, one has to perform self-consistent calculations for different number of electrons. This has been performed by several groups. Most of these calculations were based on the local density approximation of the density functional formalism (Hohenberg and Kohn, 1964; Kohn and Sham, 1965; Jones and Gunnarsson, 1989). $U_{0}$ is given by the change of the position of the $t_{1 u}$ level 
when charge is added to this level. The calculated values were 3.0 (Pederson and Quong, 1992), 2.7 (Antropov et al., 1992), 3.0 (de Coulon et al., 1992) and $3.1 \mathrm{eV}$ (Martin and Ritchie, 1993).

Estimates of $U_{0}$ can also be obtained from experimental data. One estimate is provided by

$$
U_{0}=I\left(C_{60}\right)-A\left(C_{60}\right)-E_{g}=3.3 \mathrm{eV},
$$

where $I\left(C_{60}\right)=7.6 \mathrm{eV}$ (de Vries et al., 1992) is the ionization energy, $A\left(C_{60}\right)=2.7 \mathrm{eV}$ (Hettich et al., 1991) is the affinity energy and $E_{g}=1.6 \mathrm{eV}$ (Gensterblum et al., 1991 ) is the band gap as measured by photoabsorption for a free $\mathrm{C}_{60}$ molecule. An alternative measure is given by

$$
U_{0}=I\left(C_{60}^{-}\right)-A\left(C_{60}^{-}\right) \sim 2.7 \mathrm{eV}
$$

where $I\left(C_{60}^{-}\right)=2.7 \mathrm{eV}$ (Hettich et al., 1991) is the ionization energy and and $A\left(C_{60}^{-}\right)$is the affinity energy of $C_{60}^{-}$. $C_{60}^{2-}$ has been observed experimentally (Hettich et al., 1991; Limbach et al., 1991) and if it is indeed stable, $A\left(C_{60}^{-}\right)>0$ and $U_{0}<2.7 \mathrm{eV}$. It may, however, be metastable with $A\left(C_{60}^{-}\right)$slightly negative and $U_{0}$ slightly larger than $2.7 \mathrm{eV}$. The estimate (Eq. (12)) refers to the interaction between a hole in the $h_{u}$ level and an electron in the $t_{1 u}$ level, while the estimate (Eq. (13)) refers to the interaction between two electrons added to a $\mathrm{C}_{60}$ molecule. Since the electron and hole try to stay together while the two electrons try to stay apart, it is not surprising that the latter estimate is smaller (Antropov et al., 1992).

\section{B. Coulomb $U$ for the solid}

In the solid, there are additional screening effects due to the polarization of the surrounding molecules when two electrons are added to a given molecule. In the following, we have a Hubbard model in mind, where the on-site Coulomb interaction is included, but the screening due to the polarization of the surrounding molecules is only taken into account implicitely as a renormalization of an effective $U$. In this way the Coulomb interaction for a solid was found to be $U=1.27 \mathrm{eV}$ (Pederson and Quong, 1992) and 0.8-1.3 eV (Antropov et al., 1992). The Coulomb interaction may for many purposes be drastically reduced by the metallic screening. This reduction should, however, not be included if $U$ is used in, say, a Hubbard model, since the metallic screening is then included explicitly in the model. To reduce the parameter $U$ by the metallic screening would then involve double counting.

Experimentally, $U$ has been estimated from Auger spectroscopy. Without any Coulomb interaction the
Auger spectrum would be just a convolution of the oneparticle spectrum, while the presence of an on-site molecular interaction $U$ should displace the spectrum by approximately $U$. The comparison of the Auger spectrum with the convoluted photoemission spectrum then gives an estimate of $U=1.6 \mathrm{eV}$ (Lof et al., 1992) and $U=1.4$ eV (Brühwiler et al., 1992). For the highest occupied orbital $\left(h_{u}\right)$ the estimate of $U$ is about $0.2 \mathrm{eV}$ smaller (Lof et al., 1992), and for the $t_{1 u}$ orbital it may be still somewhat smaller. Due to surface effects, Auger spectroscopy may also slightly overestimate $U$ (Antropov et al., 1992).

\section{Mott-Hubbard transition in systems with a large degeneracy}

From the estimates of $U$ and $W$ it follows that the ratio $U / W$ is substantially larger than one. It is then generally expected that the system should be a Mott-Hubbard insulator (Georges et al., 1996). It has therefore been argued that stoichiometric $\mathrm{A}_{3} \mathrm{C}_{60}$ must be an insulator and that the experimental samples of $\mathrm{A}_{3} \mathrm{C}_{60}$ are metallic only because they are nonstoichiometric (Lof et al., 1992). This would be a similar situation as for the high $T_{c}$ superconductors, and it might suggest that various properties of $\mathrm{A}_{3} \mathrm{C}_{60}$ should be understood in a similar way as for the high $T_{c}$ supersoncutors. We therefore discuss the origin of the metallicity in $\mathrm{A}_{3} \mathrm{C}_{60}$ in more detail.

The estimates of the critical value of $U / W$ for a MottHubbard transition have been made mostly for systems without orbital degeneracy, while the $t_{1 u}$ orbital has a threefold degeneracy. This was considered in early work of Lu (1994), who used the socalled Gutzwiller Ansatz and approximation, finding that the orbital degeneracy leads to a Mott-Hubbard transition for $U / W \sim(N+1)$, where $N$ is the orbital degeneracy. This strong degeneracy dependence raised questions about its qualitative explanation and about why not almost all systems with partly filled bands are metals.

To discuss qualitatively the effect of orbital degeneracy, we consider a half-filled system with the orbital degeneracy $N$ and $M$ sites. The band gap is then given by

$$
E_{g}=E(K+1)+E(K-1)-2 E(K),
$$

where $K=N M$ is the number of electrons in the neutral state and $E(K)$ is the ground-state energy for $K$ electrons. If we consider the system with $K$ electrons in the limit of a large $U$, configurations with $N$ electrons per site dominate the wave function. The hopping of an electron costs an energy $U$, and it is therefore strongly suppressed. In the state with $K+1$ electrons, the extra electron can, however, hop without an extra cost in Coulomb energy. The extra occupancy can move through the hop of any of $N$ electrons, which gives an extra factor $\sqrt{N}$ in the corresponding hopping matrix element 
compared with the one-electron case (Gunnarsson et al., 1996). This suggests that the energy is lowered by about $\sqrt{N} \varepsilon_{b}$, where $\varepsilon_{b}$ is the bottom of the one-particle band. Similar arguments for the $K$-1-electron state, suggest that

$$
E_{g}=U-\sqrt{N} W
$$

Exact diagonalization calculations and Monte Carlo calculations for $\mathrm{A}_{3} \mathrm{C}_{60}$ (Gunnarsson et al., 1996) support these conclusions and suggest that they can be extrapolated to intermediate values of $U$. Detailed calculations lead to the conclusion that the Mott-Hubbard transition takes place for $U / W \sim 2 \frac{1}{2}$. This is at the upper limit of both experimental and theoretical estimates of $U / W$. This suggests that $\mathrm{A}_{3} \mathrm{C}_{60}$ is on the metallic side of a Mott-Hubbard transition. Although experimental samples of $\mathrm{A}_{3} \mathrm{C}_{60}$ may be nonstoichiometric, there is then no need to assume so to explain why they are metallic. Since almost all physical systems have an orbital degeneracy, the degeneracy dependence of the Mott-Hubbard transition should be of interest for many other systems.

\section{COULOMB PSEUDOPOTENTIAL}

The electron-phonon interaction provides an attractive interaction between the electrons, which may be of the order $1 / 10 \mathrm{eV}$. This interaction is counteracted by the Coulomb repulsion, and two electrons on the same molecule in an undoped $\mathrm{C}_{60}$ solid may feel a repulsion of the order $U \sim 1-\frac{1}{2} \mathrm{eV}$ (see Sec. IVB). In this section we first discuss how the Coulomb repulsion may be reduced due to screening and retardation effects. A dimensionless quantity

$$
\mu=U N(0)
$$

is introduced, where $U$ is some typical (screened) interaction and $N(0)$ is the density of states per spin. Due to retardation and other effects, $\mu$ is renormalized to $\mu^{*}$, the Coulomb pseudopotential. A closely related issue is the question if the Coulomb interaction could be so effectively reduced that it becomes attractive, providing an electronic mechanism for superconductivity. This goes back to calculations for a model of a free molecule by Chakravarty et al. (1991), and is discussed in the last part of this section.

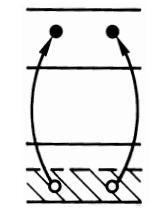

FIG. 10. Schematic picture of two subbands and the virtual Coulomb scattering of two electrons into the higher subband.

\section{A. Retardation effects. Summing ladder diagrams}

It was very early pointed out that the Coulomb repulsion can be reduced by retardation effects (Bogoliubov et al., 1958; Morel and Anderson, 1962; Schrieffer, 1964; Ginzburg and Kirzhnits, 1982). Normally, the electronic energy scale is much larger than the energy scale of the phonons. By undergoing multiple scattering into higher energy states, the electrons can move in a correlated way and thereby reduce the Coulomb interaction but still have an attractive interaction via the phonons. This is shown schematically in Fig. 10 for the scattering up into a higher subband. Mathematically, this is usually expressed by summing ladder diagrams (see Fig. 11). In simple models, e.g., assuming that all Coulomb matrix elements are equal, the ladder diagrams can be summed, giving the effective interaction (Bogoliubov et al., 1958; Morel and Anderson, 1962; Schrieffer, 1964; Ginzburg and Kirzhnits, 1982)

$$
\mu^{*}=\frac{\mu}{1+\mu \log \left(B / \omega_{p h}\right)}
$$

where $B$ is a typical electron energy (half the band width) and $\omega_{p h}$ is a typical phonon energy. If $B / \omega_{p h}>>$ $1, \mu^{*}$ can be strongly reduced relative to $\mu$. In the limit $\mu \ln \left(B / \omega_{p h}\right)>>1$, Eq. (17) simplifies to $\mu^{*} \approx$ $1 / \log \left(B / \omega_{p h}\right)$, which may be of the order $0.1-0.2$. The importance of this reduction is illustrated by the McMillan formula (McMillan, 1968) for the transition temperature

$$
T_{c}=\frac{\omega_{l n}}{1.2} \exp \left[-\frac{1.04(1+\lambda)}{\lambda-\mu^{*}(1+0.62 \lambda)}\right]
$$

In this formula, the relevant quantity is

$$
\frac{\lambda}{(1+\lambda)}-\mu^{*} \frac{1+0.62 \lambda}{1+\lambda} \approx \frac{\lambda}{(1+\lambda)}-\mu^{*}
$$

It is immediately clear that $\mu^{*}$ is as important as $\lambda$ for the $T_{c}$.

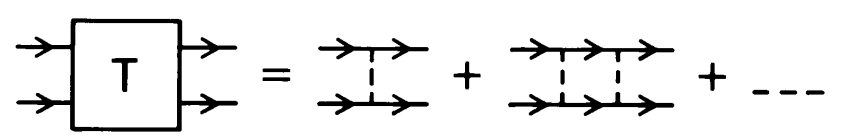

FIG. 11. Ladder diagrams describing the repeated scattering of two electrons. A full line represents an electron and a dashed line the (screened) Coulomb interaction. 
Doped $\mathrm{C}_{60}$ compounds have a number of narrow subbands with a width of about $1 / 2 \mathrm{eV}$ spread out over about $30 \mathrm{eV}$ (see Fig. 2). It has been argued that the relevant energy scale is the total width of all the subbands (Varma, 1991). Using Eq. (17) one then finds that $\mu^{*} \sim 0.2$ is only slightly larger than what is believed to be the case for conventional superconductors. Together with current estimates of $\lambda$, values of $T_{c}$ of the right order of magnitude can then easily be obtained (Varma et al., 1991; Schluter et al., 1992; Mazin et al., 1992). On the other hand, Anderson (1991) asserted that the relevant energy scale is the width of the $t_{1 u}$ subband. Since this energy is comparable to the phonon energies, the retardation effects are then expected to be small. Anderson therefore argued that $\mu^{*}$ is large, and that the phonon mechanism alone cannot be enough for explaining the superconductivity.

We first discuss the retardation effects on $\mu^{*}$ within the formalism described above, where ladder diagrams in the screened Coulomb interaction are summed. Later we discuss effects beyond this formalism. A crucial quantity is the Coulomb matrix element for scattering two electrons from the $t_{1 u}$ band into higher subbands (see Fig. 10). If these matrix elements are very small, the higher subbands should play a small role in this formalism. Chakravarty et al. (1992b) analyzed this in a model where they included the on-site interaction $U_{C}$ between two electrons on the same carbon atom and the interaction $V_{C}$ between two electrons on different carbon atoms on the same $\mathrm{C}_{60}$ molecule. They then found that the Coulomb scattering matrix element between different subbands is of the order $U_{C} / 60$, while the interaction within the same band is $U_{C} / 60+V_{C}$. Assuming that $U_{C} \approx 5-10 \mathrm{eV}$ and $V_{C} \approx 0.5-1.0 \mathrm{eV}$, they found that the interband interaction is very much smaller than the intraband interaction.

The matrix elements of the screened Coulomb interaction have been studied in the RPA (Gunnarsson and Zwicknagl, 1992; Gunnarsson et al., 1992). The system was described in a tight-binding approach (Satpathy, 1992; Laouini et al., 1995) with one $2 s$ - and three $2 p$-orbitals per carbon atom, and the screening was calculated with local-field effects included. A long-range Coulomb interaction was included, which involved an interaction between different atoms both on the same $\mathrm{C}_{60}$ molecule and on different molecules. The Coulomb interaction on a carbon atom was set to $U_{C}=12 \mathrm{eV}$. A screening calculation within the LDA approximation has been performed by D.P. Joubert (1993), with fairly similar results. The tight-binding results are shown in Table $\mathrm{X}$. The table shows both intraband and interband interactions

$$
\begin{aligned}
& W(\alpha \beta, \gamma \delta, \mathbf{q})= \\
& N \int d^{3} r d^{3} r^{\prime} \psi_{\alpha \mathbf{k}}^{*}(\mathbf{r}) \psi_{\beta \mathbf{k}+\mathbf{q}}(\mathbf{r}) W\left(\mathbf{r}, \mathbf{r}^{\prime}\right) \psi_{\gamma \mathbf{k}}\left(\mathbf{r}^{\prime}\right) \psi_{\delta \mathbf{k}+\mathbf{q}}^{*}\left(\mathbf{r}^{\prime}\right)
\end{aligned}
$$

where

$$
\psi_{\alpha \mathbf{k}}(\mathbf{r})=\frac{1}{\sqrt{N}} \sum_{\nu} e^{i \mathbf{k} \cdot \mathbf{R}_{\nu}} \varphi_{\alpha}\left(\mathbf{r}-\mathbf{R}_{\nu}\right)
$$

is a Bloch sum of molecular orbitals $\varphi_{\alpha}(\mathbf{r}), W\left(\mathbf{r}, \mathbf{r}^{\prime}\right)$ is the screened interaction and $N$ is the number of unit cells. The intraband integrals correspond to the interaction of charges which integrate to one in the unit cell, i.e., these integrals are dominated by a monopole interaction. On the other hand, $\phi_{\alpha}(\mathbf{r}) \phi_{\beta}(\mathbf{r})$ with $\alpha \neq \beta$ integrates to zero, and therefore the interband matrix elements $W(\alpha \beta, \alpha \beta)$ correspond to multipole interactions. In the solid and without screening, the intraband matrix elements are therefore very large for small $\mathbf{q}$, since they represent the interaction of monopoles placed on the different molecules and with slowly varying phase factors. When screening is introduced in the undoped solid, these integrals are reduced due to the polarization of the molecules. This reduction is much more dramatic in the doped solid due to the effective metallic screening, which involves a charge transfer between the molecules. The unscreened interband matrix elements are much smaller than the unscreened intraband elements, due to the shorter range of the multipole interaction. In the undoped solid they are further reduced, primarily due to rearrangement of the charge density inside the molecule. In the doped solid, the metallic screening does not essentially further reduce these matrix elements, since a charge transfer between the molecules does not contribute to the screening of multipoles. The moderate reduction shown in Table $\mathrm{X}$ is due to the change in the filling of the levels and the new channels (in particular, $t_{1 u} \rightarrow t_{1 g}$ ) opened up for intramolecular screening. It has been argued that the intraband (metallic) screening should not be included in the calculation of the interband matrix elements (Lammert et al., 1995). The calculations discussed above show, however, that the metallic screening of the interband matrix elements is negligible (Gunnarsson et al., 1992).

The Coulomb integral $W(\alpha \beta, \alpha \beta)$ with $\alpha \neq \beta$ referring to the $t_{1 u}$ orbitals is also of great interest, since it may tend to favor singlet states (Lammert and Rokshar, 1993). With only the short-range on-site carbon interaction $U_{C}$, it is $U_{C} / 100=0.12 \mathrm{eV}$ for a free molecule (Lammert and Rokshar, 1993). Inclusion of the longrange Coulomb interaction reduces the integral to 0.09 $\mathrm{eV}$, implying that the long range of the Coulomb interaction is not very important for this integral (in contrast to the monopole integral $W(\alpha \alpha, \alpha \alpha))$. Allowing for screening $W(\alpha \beta, \alpha \beta)$ is reduced to $0.023 \mathrm{eV}$. This value is essentially unchanged if the screening by the surrounding molecules is included. 
TABLE X. The intraband Coulomb interactions (Eq. (20))between two equal $W(\alpha \alpha, \alpha \alpha, \mathbf{q})$ and two different $W(\alpha \alpha, \beta \beta, \mathbf{q}) t_{1 u}$ conduction band Bloch states as well as the largest interband scattering matrix element $W^{\text {inter }}\left(t_{1 u} \rightarrow\right.$ $\left.t_{1 g}\right)$. We consider both a free molecule $(\mathrm{Mol})$ and a solid (Sol), where in the latter case $\mathbf{q}=\mathbf{G} / 2$ and $\mathbf{q}=\mathbf{G} / 20$, where $\mathbf{G}=2 \pi / a(1,1,1)$. The unscreened (Unscr) and screened (Scr) interactions in the undoped (Undop) and, for the solid, doped systems are shown.

\begin{tabular}{cccccccc}
\hline \hline & Sys & $W(\alpha \alpha, \alpha \alpha, \mathbf{q})$ & \multicolumn{2}{c}{$W(\alpha \alpha, \beta \beta, \mathbf{q})$} & \multicolumn{2}{c}{$W^{\text {inter }}$} \\
\hline & \multicolumn{9}{c}{$\mathbf{G} / 20$} & $\mathbf{G} / 2$ & $\mathbf{G} / 20$ & $\mathbf{G} / 2$ & $\mathbf{G} / 20$ & $\mathbf{G} / 2$ \\
\hline Unscr & Mol & \multicolumn{2}{c}{3.81} & \multicolumn{2}{c}{3.63} & 0.77 & \\
Scr & Mol & \multicolumn{2}{c}{3.72} & \multicolumn{2}{c}{3.67} & 0.14 \\
Unscr & Sol & 176.8 & 2.03 & 176.7 & 1.86 & 1.01 & 0.98 \\
Undop & Sol & 78.9 & 1.68 & 78.8 & 1.63 & 0.15 & 0.15 \\
Doped & Sol & 0.094 & 0.065 & 0.060 & 0.031 & 0.10 & 0.10 \\
\hline \hline
\end{tabular}

Because of the very efficient metallic screening of the intraband matrix elements, at least within RPA, these matrix elements are smaller than the largest interband matrix elements. These results depend crucially on the fact that these matrix elements have very different character (monopole versus multipole interaction). This behavior is also very different from the electron gas, since there is an abrupt change in character when going from one subband to another, even if they are close in energy, due to the fact that each subband is derived from a different molecular level with a different spatial character. Since Eq. (17) is derived assuming that all matrix elements are equal, it can not be used here. Approximate summing of the ladder diagrams, using the calculated matrix elements, shows that $\mu^{*}$ is close to zero in this formalism (Gunnarsson and Zwicknagl, 1992).

It is interesting to ask why the intraband matrix elements are so small. The screened interaction can be written as

$$
W=(1-v P)^{-1} v
$$

where $W$ is a matrix representing the different Coulomb matrix elements, $v$ is the corresponding unscreened matrix elements and $P$ is the polarizability, which is here calculated in the RPA. For the intraband matrix elements, the $t_{1 u}$ orbitals dominate the screening, and we can transform to the corresponding basis, neglecting all other orbitals. For small $|\mathbf{q}|$, the diagonal elements of $P$ are then related to the density of states $N(0)$ per spin. Since both $N(0)$ and the diagonal elements of $v$ are large, we can assume that the product is much larger than unity. Essentially, the two factors $v$ in Eq. (22) then drop out, and with appropriate assumptions one can then derive (Gunnarsson et al., 1992) that the intraband matrix elements are

$$
\frac{1}{3} W(\alpha \alpha, \alpha \alpha, \mathbf{q})+\frac{2}{3} W(\alpha \alpha, \beta \beta, \mathbf{q}) \approx \frac{1}{2 N(0)},
$$

for small $|\mathbf{q}|$. To obtain the unrenormalized $\mu$ we multiply by $N(0)$ obtaining $\mu \approx 0.5$. Averaging over $q$ gives $\mu \approx 0.4$. We note that $W$ becomes very small in the RPA for large $N(0)$, since in the RPA the cost of screening is purely a kinetic energy cost, and for a large $N(0)$ this cost is very small. For large values of $U$, we expect RPA to become a poor approximation, and it is therefore an interesting and important question how accurate RPA is in this case.

\section{B. Beyond ladder diagrams}

The considerations above were all within a framework where ladder diagrams in the screened interaction are summed. This raises the question about the validity of neglecting other diagrams. For instance, Grabowski and Sham (1984) studied the lowest order vertex corrections for the electron gas and found important corrections. Gunnarsson et al. (1992) constructed other sets of diagrams, which to a large extent cancelled the ladder diagrams. This raises important questions about which diagrams to include.

To circumvent this problem, Gunnarsson and Zwicknagl (1992) and Gunnarsson et al. (1992) studied a twoband model where some exact results can be obtained. Thus they considered the Hamiltonian

$$
\begin{aligned}
& H=\sum_{i} \sum_{n=1}^{2} \sum_{\sigma} \varepsilon_{n} n_{i n \sigma}+t \sum_{<i j>} \sum_{n=1}^{2} \sum_{\sigma} \psi_{i n \sigma}^{\dagger} \psi_{j n \sigma} \\
& +U_{11} \sum_{i} \sum_{n n^{\prime} \sigma \sigma^{\prime}}{ }^{\prime} n_{i n \sigma} n_{i n^{\prime} \sigma^{\prime}} \\
& +U_{12} \sum_{i}\left[\psi_{i 2 \uparrow}^{\dagger} \psi_{i 2 \downarrow}^{\dagger} \psi_{i 1 \downarrow} \psi_{i 1 \uparrow}+\text { h.c. }\right],
\end{aligned}
$$

where $i$ is a site index, $\varepsilon_{n}$ the energies of the two levels, $t$ is the hopping integral, $U_{11}$ is the intraband interaction and $U_{12}$ is the interband scattering. The particularly strong interband scattering between the $t_{1 u}$ and $t_{1 g}$ bands in $\mathrm{C}_{60}$ is of a similar type, namely the excitation of two electrons between two bands (see Fig. 10). It was then assumed that

$$
\Delta \varepsilon \equiv \varepsilon_{2}-\varepsilon_{1}>>U_{12}, t .
$$

In this limit, the upper subband can be projected out and a new effective Hamiltonian be obtained, which describes low-energy properties correctly. This process does not generate any new terms for the Hamiltonian (24), but it renormalizes the intraband interaction to

$$
U_{e f f}=U_{11}-\frac{U_{12}^{2}}{2 \Delta \varepsilon} .
$$

If it is now assumed that $U_{12}^{2} /(2 \Delta \varepsilon)<<U_{11}$, the renormalization of the intraband interaction is small. The 
properties of this effective Hamiltonian then differ very little from the one-band model, where the upper band was completely neglected. Thus the upper band has a small influence on the low energy properties in general and $\mu^{*}$ in particular. For instance, in RPA the screened interaction is $1 /[2 N(0)]$ in both the one-band and twoband models, if $U_{11} N(0)>>1$.

On the other hand, the ladder diagrams in the screened interaction due to scattering into the upper subband can be summed. If it is assumed that

$$
\frac{1}{N(0)} \lesssim \frac{U_{12}^{2}}{2 \Delta \varepsilon}
$$

this approach predicts a large renormalization of $\mu^{*}$. The ladder diagrams subtract a quantity $U_{12}^{2} /(2 \Delta \varepsilon)$, which, although small compared with $U_{11}$, is large compared with the screened interaction in the limit considered. Thus the summation of ladder diagrams leads to a qualitatively incorrect result in this case.

The projection method and the summation of ladder diagrams describe similar physics. The difference is that in the rigorous projection method, the high energy degrees of freedom were eliminated first, while in the ladder diagram approach the low energy degrees of freedom were treated first (by introducing the metallic screening) and the high energy degrees of freedom later (by summing the ladder diagrams). These results should be of relevance for the retardation effects due to the high-lying states also for conventional superconductors.

The model above contains important features of $\mathrm{A}_{3} \mathrm{C}_{60}$ and the assumptions are not too unrealistic for this system. The results therefore suggest that the summation of ladder diagrams in the screened interaction gives unreliable results for $\mathrm{A}_{3} \mathrm{C}_{60}$ and that the renormalization of $\mu^{*}$ due to the higher subbands may not be large. For simplicity one may therefore assume that this renormalization can be neglected completely. If in addition RPA screening is used, one arrives at $\mu^{*} \sim 0.4$. The approach above has only addressed the renormalization due to the higher subbands but not due to processes within the $t_{1 u}$ band itself. If we assume that the traditional theory is valid for these processes, $\mu^{*}$ is renormalized to 0.3 according to formula (17), if we set $B=0.25 \mathrm{eV}$ and $\omega_{p h}=0.1 \mathrm{eV}$. Such a value is then to be used in the McMillan formula, while the value 0.4 applies to the Eliashberg equation including one subband. We notice that only static screening was considered above, although inclusion of dynamic effects may influence the results substantially (Rietschel and Sham, 1983).

A very interesting work was performed by Takada (1993). He considered a model including electrons in the $t_{1 u}$ band, phonons and plasmons, where the plasmons screened the strong Coulomb repulsion. Guided by the Ward (1950) identity and work by Nambu (1960), Takada used an approximate vertex correction. In this way he was able to include corrections to Migdal's theorem, vertex corrections to the electron-electron scattering in the $t_{1 u}$ band and the dynamical screening of the Coulomb interaction. It was found that the corrections to Migdal's theorem enhanced the value of $T_{c}$ for moderate values of the phonon frequency. Furthermore it was found that the vertex corrections to the electron-electron scattering reduce $T_{c}$. Thus there is a partial cancelation of the enhancement (Rietschel and Sham, 1983) of $T_{c}$ due to dynamic screening effects, as had also been found earlier (Grabowski and Sham, 1984). Takada used the value $\mu=0.4$ for the Coulomb pseudopotential derived above, to describe static screening effects and the renormalization due to scattering in other sub bands than the $t_{1 u}$ band. Using $\lambda=0.58$ and adjusting the electronplasmon coupling, Takada (1993) could then reproduce the experimental $T_{c}$ for $\mathrm{K}_{3} \mathrm{C}_{60}$. The corresponding isotope effect $\alpha=0.15$ for $\mathrm{K}_{3} \mathrm{C}_{60}$ was, however, surprisingly small. To describe the lattice parameter dependence of $T_{c}$, Takada had to assume that $\mu=0.4 W_{0} / W$, where $W_{0}$ and $W$ are the band widths of $\mathrm{K}_{3} \mathrm{C}_{60}$ and the system studied, respectively. This is somewhat surprising, since in RPA $\mu$ is predicted to be essentially independent of the lattice parameter.

\section{Electronic mechanisms}

Chakravarty and Kivelson (1991), Chakravarty et al. (1991) and Baskaran and Tosatti (1991) proposed that there may be an effective attractive interaction between the electrons for a $\mathrm{C}_{60}$ molecule of a purely electronic origin. Chakravarty and Kivelson (1991) introduced a Hubbard model of the $\pi$-electron system in a free molecule with an on-site interaction $U_{C}$, only. Using second order perturbation theory, they calculated the energy of $\mathrm{C}_{60}$, $\mathrm{C}_{60}^{-}$and $\mathrm{C}_{60}^{2-}$. This provided an estimate of the effective repulsion for $\mathrm{C}_{60}^{-}$. When the singlet state of $\mathrm{C}_{60}^{2-}$ was considered, the result consisted of a positive, linear term and a negative, quadratic term in $U_{C}$. The interaction therefore becomes attractive for sufficiently large $U_{C} \sim 3 t_{0}$, where $t_{0}$ is the intramolecular hopping. The tendency to the formation of a singlet state results from an integral of the type $W(\alpha \beta, \alpha \beta)$ (see Eq. (20)) with $\alpha \neq \beta$ belonging to the $t_{1 u}$ band (Lammert and Rokhsar, 1993), which may become negative when the higher states are projected out in second order perturbation theory. The following discussion has focussed on the validity of the second order perturbation theory and the neglect of a long-range Coulomb interaction in this type of work.

White et al. (1992) addressed the question about the validity of second order perturbation theory, by studying small model systems. In particular, they considered a model with twelve atoms located on a truncated tetrahedron. For this system exact diagonalization can be performed and compared with second order perturbation 
theory. White et al. (1992) concluded that second order perturbation theory is qualitatively correct for intermediate values of $U_{C}$ in the sense that exact diagonalization also gives an attractive interaction, but that second order perturbation theory may greatly overestimate the magnitude. Auerbach and Murthy (1992) and Murthy and Auerbach (1992) studied a model with electrons on a sphere and with an interaction of variable range. They calculated the second order contribution to pairing and they also estimated the third order terms. The second and third order contributions were found to be comparable at an interaction strength which was substantially smaller than the strength giving pairing in the second order calculation. This again raises doubts about the accuracy of the second order calculation. Berdenis and Murthy (1995) performed a renormalization group calculation for a Hubbard model of $\mathrm{C}_{60}$ using a method of Tokuyasu et al. (1993). This method remains accurate up to larger values of the atomic $U_{C}$ than the second order perturbation theory, but its accuracy becomes questionable at roughly the value of $U_{C}$ where it predicts an attractive interaction. Thus it seems to still be unclear whether or not the interaction can be attractive for an on-site Hubbard model of a free $\mathrm{C}_{60}$ molecule.

Goff and Phillips (1992; 1993) and Auerbach and Murthy (1992) have studied the effective interaction as a function of the range of the interaction. They found that the use of a more long-ranged interaction tended to suppress the formation of an attractive effective interaction. With an on-site interaction, two electrons added to the $t_{1 u}$ orbitals can almost avoid being on the same site simultaneously and they have only a weak direct interaction $W(\alpha \alpha, \alpha \alpha)$. It is then not entirely surprising that the renormalized interaction $W(\alpha \beta, \alpha \beta)$ can lead to an effective attractive interaction. If the interaction becomes more long-ranged, the direct interaction rapidly increases, leading to a repulsive effective interaction. For the free molecule, the interaction is long-range and there is actually an "anti-screening" effect for electrons on the opposite side of the molecule (Gunnarsson et al., 1992). The reason is that for a finite system, screening charge cannot be moved to infinity as for an infinite system. It is therefore no surprise that the effective interaction for the free molecule is large and repulsive, as discussed in Sec. IV.A. Chakravarty et al. (1991) therefore from the start pointed out that the attractive electronic mechanism could become operative only if the screening inside the molecule is much more efficient in the metal than in the free molecule.

Lammert et al. (1995) considered a model with a long-range Coulomb interaction. It was argued that the molecules surrounding a specific molecule provide a metallic environment, which was approximated by a metal with a spherical cavity with the radius $R_{c}$. In this way the corresponding degrees of freedom were integrated out first, although the metallic screening corresponds to a low energy scale in the problem $\left(W \sim \omega_{p l} \sim 0.5 \mathrm{eV}\right.$ (Knupfer et al., 1995)). The resulting screened interaction was calculated and used for the low-frequency interactions in the problem. The effective singlet two-body interaction was then calculated diagrammatically, including the the first and second order diagrams. An attractive interaction was obtained for $R_{c}$ in the range 5-6 $\AA$ and the on-site carbon $U_{C}$ in the range $8-10 \mathrm{eV}$.

An alternative way of treating the problem within this spirit, is to express the metallically screened interaction $U_{m e t . s c r}$. in terms of the Coulomb interaction $U_{0}$ between two electrons on a free $\mathrm{C}_{60}$ molecule. It is first assumed that in the solid the addition of a charge $q$ to a molecule induces a potential $-q V_{0}$ due to the metallic screening by the surrounding molecules, reducing $U_{0}$ to

$$
U_{m e t . s c r .}=U_{0}-V_{0}
$$

in the solid. We here assume that due to the small hopping between the $\mathrm{C}_{60}$ molecules, the surrounding molecules respond to a spherical symmetric average of the charge on the central molecule. In the model of Lammert et al. (1995), replacing the surrounding by a spherical cavity, $V_{0}=e^{2} / R_{c}$. To obtain an estimate of $V_{0}$ (or $R_{c}$ ) in the above spirit, one can treat the surrounding $\mathrm{C}_{60}$ molecules as grounded, metallic spheres with the radius $4.46 \AA$ (which reproduces the experimental molecular polarizability). This leads to $V_{0}=2.2 \mathrm{eV}$ (Krier and Gunnarsson, 1995), corresponding to $R_{c}=6.6 \AA$, i.e., too large to obtain an attractive interaction in the work of Lammert et al. (1995). If this is combined with the estimate of $U_{0} \sim 2.7 \mathrm{eV}$ (see Sec. IVA), a large and positive $U_{\text {met.scr. }} \sim 0.5 \mathrm{eV}(\mu=N(0) U \sim 4)$ results. This may suggest that it is important to also allow for charge transfer to the molecule where the studied electrons are located, as normally assumed for metallic systems.

An alternative electronic mechanism was suggested by Friedberg et al. (1992). They argued that it is favorable to form a two-electron wave-function involving one $t_{1 u}$ and one $t_{1 g}$ state. In this way a correlated state can be formed, which lowers the Coulomb energy. Friedberg et al. (1992) argued that this more than outweighs the cost in one-electron energy. This two-electron state is bosonlike and forms a Bose band. It was suggested that this may lead to a Bose-Einstein condensate.

\section{BEYOND THE MIGDAL-ELIASHBERG THEORY}

\section{A. Migdal's theorem}

Migdal's theorem states that vertex corrections in the electron-phonon interaction can be neglected if the typical phonon frequencies $\omega_{p h}$ are sufficiently much smaller than the electronic energy scale, say the Fermi energy $E_{F}$ (Migdal, 1958). This theorem is assumed to be valid 
in the derivation of the Eliashberg (1960) theory. For the fullerides, the highest phonon frequency is about 0.2 $\mathrm{eV}$ and the width of the $t_{1 u}$ band is about $1 / 2 \mathrm{eV}$, giving $E_{F} \sim 0.25 \mathrm{eV}$. Migdal's theorem may therefore be strongly violated for the fullerides. If higher sub bands are included, one may argue that $E_{F}$ is much larger, but the conclusion that Migdal's theorem is violated remains correct (Gunnarsson et al., 1994b).

The lowest order vertex corrections have been studied by Grabowski and Sham (1984), who calculated these corrections approximately, finding that they can strongly reduce $T_{c}$. More recently, the issue has been studied extensively by Pietronero and coworkers (Pietronero and Strässler, 1992; Pietronero et al., 1995; Grimaldi et al., $1995 \mathrm{ab})$. They introduced a theory which is nonperturbative in $\lambda$ and perturbative in $m \lambda$, where $m=\omega_{p h} / E_{F}$.
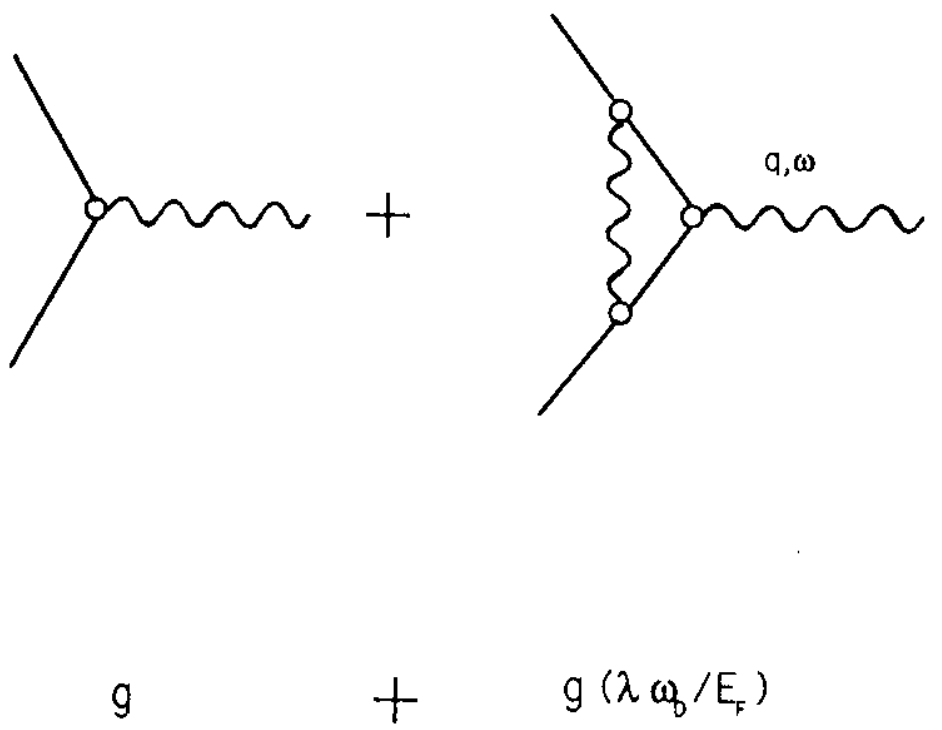

FIG. 12. The zeroth order vertex and the first order vertex correction. (After Pietronero et al., 1995)

First the lowest order vertex correction was calculated (see Fig. 12) for an electron gas like model. The result is shown in Fig. 13 for $m=1$ and as a function of $\omega$ and $Q=q /\left(2 k_{F}\right)$, where $\omega$ and $q$ are the Matsubara frequency and wave vector of the phonon line and $k_{F}$ is the Fermi wave vector. The energy of the incoming electron was set to zero, and $\omega_{0}=\omega_{p h}$ is the energy of the Einstein phonon. This should be compared with the zeroth order contribution, which is unity. The vertex correction has a very strong $Q$ dependence. For small $Q \neq 0$ and small $\omega$ it is large and negative, while it is positive elsewhere. This suggests that the influence on $T_{c}$ may depend strongly on the system. An Eliashberg-like theory was then developed, including the lowest order vertex correction and two "crossed" phonon lines in the scattering between two electrons (Grimaldi et al.. 1995ab). Fig.
14 shows their results for $T_{c}$ and for the isotope effect

$$
\alpha=-\frac{d \ln T_{c}}{d \ln M},
$$

where $M$ is the carbon mass. These results were calculated assuming that there is a cut off in $q$-space at some critical $Q_{c}$. It was argued (Grimaldi et al., 1995ab) that this cut off may simulate specific one-particle effects or correlation effects (Kulic and Zheyer, 1994). Depending on the value of $Q_{c}$, the theory may predict a large enhancement of $T_{c}$ or no enhancement at all. The value of the isotope effect can also vary substantially, and even be larger than 0.5. In the limit where Migdal's theorem is valid, this theory gives $\alpha=0.5$, since no Coulomb effects are included $\left(\mu^{*}=0\right)$.

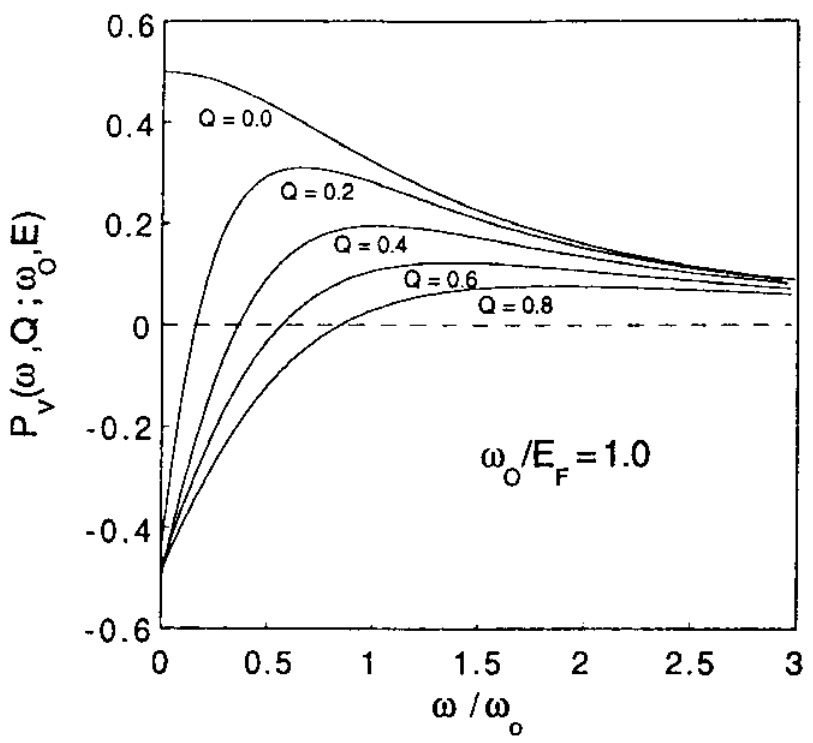

FIG. 13. Behavior of the vertex correction for different values of $Q \equiv q /\left(2 k_{F}\right)$. (After Pietronero et al., 1995).

As described in Sec. Vi. Takada (1993) also considered corrections to Migdal's theorem, and found that these enhance $T_{c}$ for moderate values of the phonon frequencies. Ikeda et al. (1992) showed that Migdal's theorem becomes valid again when the phonon energies are much larger than the electronic energies. Kostur and Mitrovic (1993) studied the vertex corrections with the emphasis on two-dimensional systems and Krishnamurthy et al. (1994) and Cappelluti and Pietronero (1996) studied systems with a van Hove singularity. Kostur and Mitrovic (1994) studied the corrections to $T_{c}$ from vertex corrections for several model interactions. For a BCS-type of interaction and an isotropic electron-phonon interaction, they found that vertex corrections reduce $T_{c}$, while for a highly anisotropic interaction, inspired by the cuprate superconductors, they found that $T_{c}$ can also be enhanced by the vertex corrections. Asai and Kawaguchi (1992) argued that nonadiabatic effect could enhance the coupling 
by $10-30 \%$.

The violation of Migdal's theorem is also expected to play an important role for the photoemission spectrum. According to Migdal's theorem, the spectrum should just show single phonon satellites. Due to various broadening effects, this cannot be checked experimentally for $\mathrm{A}_{3} \mathrm{C}_{60}$. Theoretical calculations suggest, however, that multiple satellites develop (Gunnarsson et al., 1994ab). This involves a substantial transfer of spectral weight to higher binding energies, and the coupling to phonons and plasmons may explain (Knupfer et al., 1993) the large observed width of the $t_{1 u}$ band in photoemission (Chen et al., 1991). . The work above illustrates that the violation of Migdal's theorem could have important effects for the fullerides, but the magnitude, and perhaps even the sign, of the corrections seem to be not fully established.
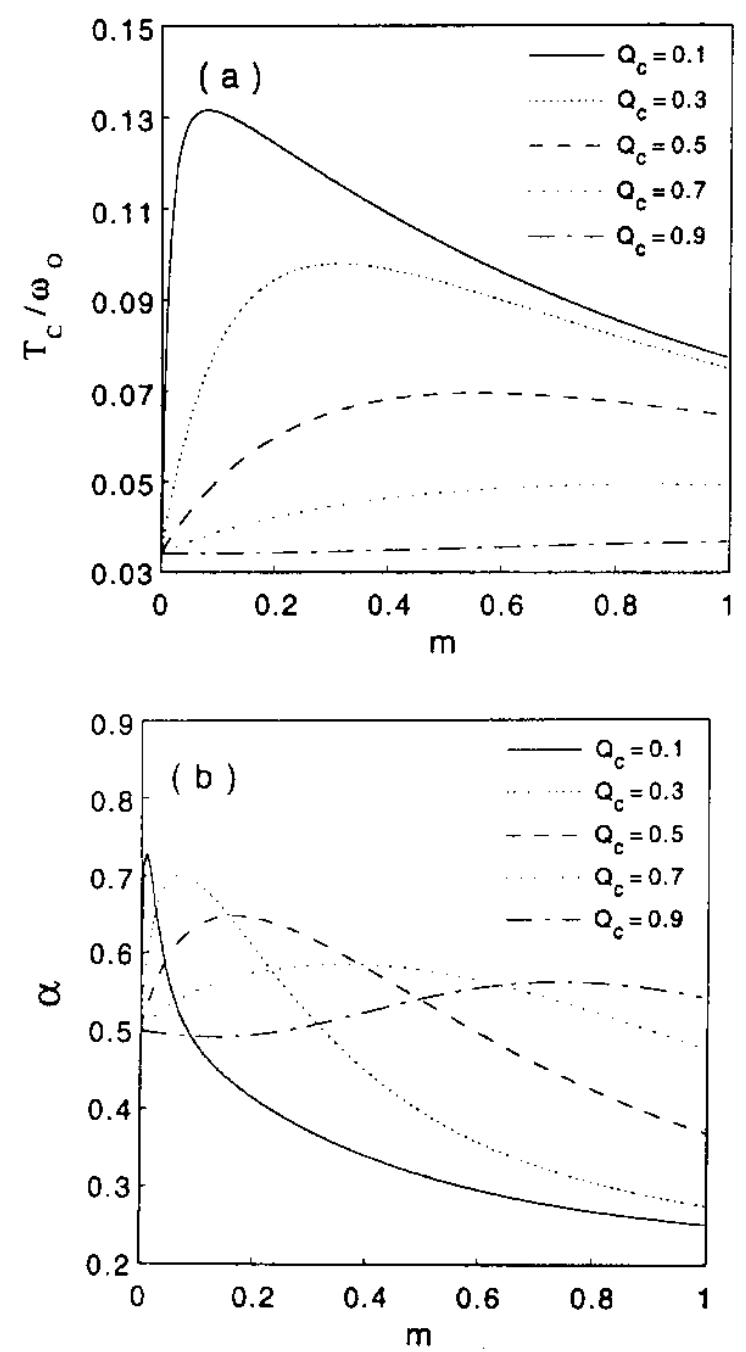

FIG. 14. (a) $T_{c}$ as a function of $m \equiv \omega_{p h} / E_{F}$ and the cut off momentum $Q_{c}$ for $\lambda=0.5$. (b) Isotope effect exponent $\alpha$ as a function of $m$ and $Q_{c}$. (After Grimaldi et al., 1995).

\section{B. Jahn-Teller effects beyond Migdal-Eliashberg treatment}

The three-fold degeneracy of the $t_{1 u}$-level is lifted when a vibration of $\mathrm{H}_{g}$ symmetry is excited. This makes $\mathrm{C}_{60}$ molecules with a partly filled $t_{1 u}$ level unstable to a JahnTeller distortion (Jahn and Teller, 1937). It has been shown (Ihm, 1994; Auerbach et al., 1994; Manini et al., 1994) that this Jahn-Teller system has a Berry phase (Berry, 1984), which imposes selection rules on the allowed transitions. These Jahn-Teller modes are included in the Migdal-Eliashberg theory under the assumption that the band width is much larger than the phonon frequency. Below we discuss how in the opposite limit, the molecular limit, some new interesting effects appear. These effects may possibly be relevant also for doped $\mathrm{C}_{60}$ compounds, although the band width may be too large for these systems to allow the survival of the molecular properties.

It is interesting to study the energy of $\mathrm{C}_{60}^{n-}$ as a function of the electron-phonon coupling strength. A JahnTeller energy is defined as

$$
E_{J T}=\sum_{\nu=1}^{8} \frac{g_{\nu}^{2}}{\omega_{\nu}}
$$

where the sum is over the eight $\mathrm{H}_{g}$ modes and $g_{\nu}$ and $\omega_{\nu}$ are the coupling strength and energy, respectively, of the $\nu$ th mode. In the strong-coupling limit, the energy can then be written as (Lannoo et al., 1991)

$$
E^{S C}(n)=C(n) E_{J T}
$$

where $C(n)=1(n=1,5), 4(n=2,4)$ or $3(n=3)$. It was emphasized by Yabana and Bertsch (1992), Auerbach et al. (1994) and Manini et al. (1994) that in the weak-coupling limit the prefactor in the energy expression is a factor $5 / 2$ larger

$$
E^{W C}(n)=\frac{5}{2} C(n) E_{J T},
$$

due to the Jahn-Teller effect, while for a mode of $\mathrm{A}_{g}$ symmetry the two expressions (31) and (32) are identical. This result is valid for a free molecule or a solid with zero band width, while the Migdal-Eliashberg theory is valid in the opposite limit of a large band width. Auerbach et al. (1994) and Manini et al. (1994) observed that in the zero band width, weak-coupling limit, the attractive interaction between two electrons due the the electron-phonon interaction for $\mathrm{C}_{60}^{3-}$,

$$
U_{p h}^{W C}(3)=-5 E_{J T},
$$

is a factor of three larger than in the Eliashberg theory. This could potentially have important implications for the superconductivity. We notice, however, that for realistic estimates of the coupling constants, the system 
is not in the weak-coupling limit and may actually be closer to the strong-coupling limit (Gunnarsson, 1995). The prefactor in Eq. $(32,33)$ is then strongly reduced already in the zero band width limit.

\section{CALCULATED PROPERTIES}

\section{A. Transition temperature}

It was very early pointed out that the estimated values of the electron-phonon coupling $\lambda$ to the intramolecular modes are of the right order of magnitude to explain the transition temperature $T_{c}$ (Varma et al., 1991; Schluter et al., 1992; Mazin et al., 1992). Since the calculated values of $\lambda$ are only intermediate, it is important for the explanation of the rather high values of $T_{c}$ that the intramolecular phonon frequency are quite high. For values of $\lambda$ in the upper range of the estimates, the rather large values of $T_{c}$ can be understood even if it is assumed that the inefficient renormalization of $\mu^{*}$ by retardation effects in the fullerides leads to substantially larger $\mu^{*}$ than for conventional superconductors. For instance, Gunnarsson et al. (1995) solved the Eliashberg equation, using the coupling constants deduced from photoemission for a free $\mathrm{C}_{60}^{-}$molecules (see Sec. III.A.4), a $t_{1 u}$ band with the width $1 / 2 \mathrm{eV}$ and the density of states at the Fermi energy deduced from NMR (see, Sec. III.A.4). It was found that to reproduce the values of $T_{c}$ for $\mathrm{K}_{3} \mathrm{C}_{60}$ and $\mathrm{Rb}_{3} \mathrm{C}_{60}, \mu^{*}$ had to be assumed to be 0.6, i.e., even larger than the estimate in Sec. V.B. We note that the value 0.6 used in the Eliashberg equation corresponds to the value 0.4 in the McMillan formula, due to renormalization effects inside the $t_{1 u}$ band. From the discussion in the previous sections it is, however, clear that the uncertainties in the estimates of $\lambda$ and $\mu^{*}$ are substantial, and the use of the Migdal-Eliashberg theory introduces further uncertainties.

Zheng and Bennemann (1992) studied effects of the phonon energies being comparable to the band width. They used the Eliashberg theory, i.e., Migdal's theorem was implicitly assumed to be valid, but they did not assume the band width to be large in the solution of the Eliashberg theory. As a result they found that the quasiparticle weight was less renormalized than is normally assumed (the weight was larger than $1 /(1+\lambda)$ ). As a result their calculated value of $T_{c}$ was larger than according to, e.g., the McMillan (1968) formula.

\section{Lattice parameter dependence}

As discussed in Sec. II.A, for the $\mathrm{A}_{3} \mathrm{C}_{60}$ superconductors, except $\mathrm{Cs}_{3} \mathrm{C}_{60}, T_{c}$ increases with the lattice parameters. The explanation of this behavior was provided very early (Fleming et al., 1991; Zhou et al., 1992; Varma et al., 1991; Schluter et al., 1992; Mazin et al., 1992). As discussed in Sec. III.A.2, for the coupling to the intramolecular phonons $\lambda=V N(0)$ can to a very good approximation be factorized in an intramolecular part $V$ and the density of states $N(0)$ (Lannoo et al., 1991). The intramolecular part $V$ can be expected to be essentially independent of the lattice parameter $a$, while $N(0)$ grows with $a$. This follows, since as $a$ increases, the overlap between the molecules is reduced, leading to a reduced band width and an increased density of states. Fig. 15 shows a calculation of $T_{c}$ based on a tight-binding calculation of the $N(0)$, assuming a power law reduction of the hopping matrix elements with the separation between the closest atoms on neighboring molecules (Schluter et al., $1992 \mathrm{~b})$. The exponent of the power law was adjusted to experiment.

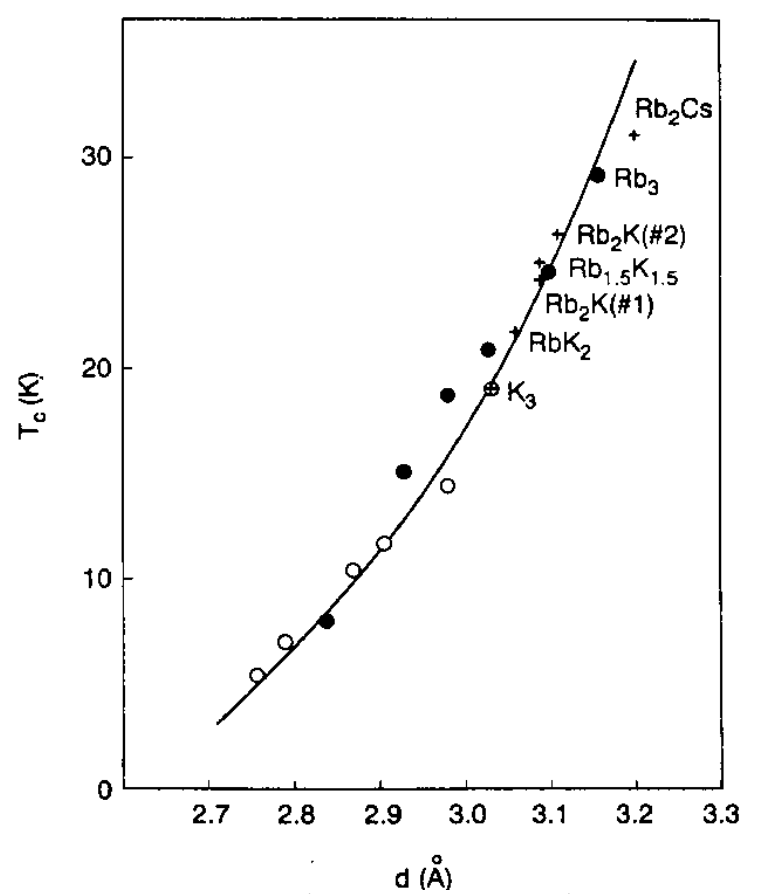

FIG. 15. Experimental (circles and crosses) and theoretical (full line) variation of $T_{c}$ with the shortest distance $d$ between two closest carbon atoms on neighboring $\mathrm{C}_{60}$ molecules. The density of states was assumed to have a power law dependence on $d, N(0) \sim d^{-n}$, with $n=2.7$ adjusted to experiment. (After Schluter et al., (1992b))

The very strong variation of $T_{c}$ with the lattice parameter $a$ in $\mathrm{Na}_{2} \mathrm{AC}_{60}$ (see Fig. 3) suggests a strong variation of $N(0)$ with $a$ in this system (see, e.g., Yildirim et al., 1995). Indeed, Maniwa et al. (1995) deduced $N(0)$ from the NMR relaxation rate for $\mathrm{Na}_{2} \mathrm{AC}_{60}(\mathrm{~A}=\mathrm{K}, \mathrm{Rb}, \mathrm{Cs})$ at ambient pressure and found a much stronger dependence than for the Fm $\overline{3} \mathrm{~m}$ structure, although possibly not quite strong enough to fully explain the strong variation of $T_{c}$. Simple tight-binding calculations including 60 "radial" p-orbital per $\mathrm{C}_{60}$ molecule did, however, not give 
a very different $a$-dependence for the density of states for the two structures (Gunnarsson, 1993). It has instead been suggested that $\mathrm{Na}_{2} \mathrm{KC}_{60}$ and $\mathrm{Na}_{2} \mathrm{RbC}_{60}$ may have structural distortions, and that these distortions possibly could reduce $N(0)$ much more than the change in the lattice parameter (Maniwa, et al., 1995). Since the Fermi energy falls fairly close to a maximum in the density of states in the undistorted Pa $\overline{3}$ structure (Satpathy et al., 1992), such a reduction is possible. An intermediate $a$-dependence was observed in pressure experiments for $\mathrm{Na}_{2} \mathrm{CsC}_{60}$ (Mizuki, 1994), possibly consistent with the observation that the structure may also distort under pressure (Zhu, 1995; Fischer, 1996). Whether $N(0)$ in the ideal structure has a strong dependence on $a$ or the strong dependence could be explained by other factors remains an interesting issue. For instance, it has also been proposed that paramagnetic impurities may suppress $T_{c}$ in $\mathrm{Na}_{2} \mathrm{KC}_{60}$ and $\mathrm{Na}_{2} \mathrm{RbC}_{60}$ (Tanigaki and Prassides, 1995).

\section{Doping dependence}

As discussed in Sec. II.A, Yildirim et al. (1995, 1996ab) have found a strong doping $(n)$ dependence for $T_{c}$, by studying $\mathrm{Na}_{2} \mathrm{Cs}_{x} \mathrm{C}_{60}(n \leq 3)$ and $\mathrm{Rb}_{3-x} \mathrm{Ba}_{x} \mathrm{C}_{60}$ $(n \geq 3)$. They found a maximum for $T_{c}$ close to halffilling $(n=3)$ and rapid decrease as $n$ was reduced or increased. The reduction for $n>3$ may be explained by considering the density of states for a system with merohedral disorder (Yildirim et al., 1995). The density of states shows a steady reduction with energy, except at the bottom of the band (Gelfand and Lu, 1992). As the filling $n$ is increased, the density of states at the Fermi energy $N(0)$ then drops, and $\lambda$ is reduced, which may explain the drop in $T_{c}$. It appears, however, that there is no such explanation for $n<3$. A tight-binding calculation for the appropriate structure ( $\mathrm{Pa} \overline{3})$ shows an increase in the density of states as the energy is lowered (Satpathy et al., 1992). The situation is changed slightly if the potential from the alkali atoms is taken into account (Gunnarsson, 1996). The nearest alkali neighbors of a given $\mathrm{C}_{60}$ molecule set up a potential with $l=4$, which cannot be efficiently screened by the $\mathrm{C}_{60}$ molecule, and which modifies the density of states. If it is taken into account, however, that the density of states is sampled over a finite energy, due to the rather large phonon energies, the basic tendency of the density of states to grow when the energy is reduced remains. One might have expected the potential of the vacancies to strongly influence the density of states. The potential from the vacancies has, however, mainly monopole or dipole character relative to a neighboring $\mathrm{C}_{60}$ molecules, and it is therefore efficiently screened. Thus the drop in $T_{c}$ as $n$ is reduced appears not to be understood.

\section{B. Isotope effect}

As discussed in Sec. II.C different experiment give widely different results for the isotope effect $\left(T_{c} \sim M^{-\alpha}\right)$, with $\alpha$ even being larger than unity. In the case of almost complete substitution $\alpha=0.30$ was found for $\mathrm{K}_{3} \mathrm{C}_{60}$ and $\mathrm{Rb}_{3} \mathrm{C}_{60}$ (Chen and Lieber, 1992).

It was pointed out early that a reasonable value of $\alpha \sim 0.3$ can be obtained if the mechanism for superconductivity is assumed to be the interaction with the intramolecular phonons (Schluter, 1992b). For instance, from the McMillan formula (1), the prefactor $\omega_{p h}$ gives $\alpha=0.5$ and the renormalization of $\mu^{*}$ as defined in Eq. (17) gives a reduction of $\alpha$. Thus $\alpha$ is reduced to about 0.3 if it is assumed that $\mu^{*} \sim 0.2$ and $\omega_{p h} \sim 1000 \mathrm{~cm}^{-1}$. We note, however, that the McMillan formula is not very accurate for calculating the isotope effect. The isotope effect was also calculated in the model discussed in the previous (VII.A) section using the Eliashberg theory and the coupling constants derived from photoemission (Gunnarsson et al., 1995). The values of $\alpha$ were then found to be 0.32 and 0.37 for $\mathrm{K}_{3} \mathrm{C}_{60}$ and $\mathrm{Rb}_{3} \mathrm{C}_{60}$, respectively.

Deaven and Rokhsar (1993) addressed the large spread in the experimentally observed isotope effect $\alpha$ for different isotope substitions. They considered a model with harmonic phonons and a linear electron phonon coupling. Within the Migdal-Eliashberg framework, they showed that the anomolous isotope effect cannot be explained. In particular, they showed that $\lambda$ is independent of the isotope distribution. Anharmonic effects have been considered by, e.g., Crespi et al. (1991) and Inada and Nasu (1992). These effects could in principle lead to $\alpha>0.5$.

The isotope effect has traditionally been considered as a sign of an electron-phonon mechanism. It has, however, been pointed out that even for an electronic mechanism, there is an isotope effect due to the change of the electronic structure by zero-point vibrations (Chakravarty et al., 1992a, Ashcroft and Cyrot, 1993). Ashcroft and Cyrot (1993) emphasized the opposing effects of the translational phonons and the librations on the electronic structure. They argued that an increase in the carbon mass would tend to reduce the hopping integral due to the translational vibrations but increase it due to the librations. They found that depending on the precise assumptions about the parameters, $\alpha$ could take a broad range of values, including values larger than 0.5 . In view of this it is not clear how much is proven by the reasonable values for $\alpha$ found from the intramolecular phonons alone.

\section{Reduced gap}

The superconductivity gap $\Delta$ is of interest, since it may give indications about strong-coupling effects. Gunnarsson et al., (1995) calculated the gap using the Eliashberg 
equation in the model discussed above, using the couplings deduced from photoemission. They obtained the values $2 \Delta / T_{c}=3.59$ and 3.66 for $\mathrm{K}_{3} \mathrm{C}_{60}$ and $\mathrm{Rb}_{3} \mathrm{C}_{60}$, respectively. This is close to the $\mathrm{BCS}$ value 3.52 and in agreement with most recent measurements.

The much larger values found in point contact tunneling measurements have stimulated a substantial amount of work. Mazin et al. (1993b) studied a model with coupling to both intramolecular phonons (with frequencies around $1000 \mathrm{~cm}^{-1}$ ) and to low-lying modes with frequencies of the order $40 \mathrm{~cm}^{-1}$. The latter modes could be librations, intermolecular or alkali modes. Due to the huge difference between the frequencies, this model shows very interesting properties. The low-lying modes hardly influence $T_{c}$ but have a strong influence on the reduced gap $2 \Delta / T_{c}$. Thus Mazin et al. (1993b) could use typical couplings to the intramolecular modes $\left(\lambda_{1} \sim 0.5\right)$ to produce the measured values of $T_{c}$, but at the same time use strong coupling to the low-lying modes $\left(\lambda_{2} \sim 2.7\right)$ to produce a large reduced gap $\left(2 \Delta / T_{c} \sim 5\right)$. More recent work has suggested that the coupling to the low-lying modes is much weaker (see Sec. III.B, C and D) and that the reduced gap may be much smaller than found in point contact tunneling. The model of Mazin et al. (1993b) may therefore not be applicable to $\mathrm{A}_{3} \mathrm{C}_{60}$, but it is still a nice illustration of the unconventional properties that can be obtained if bosons of very different energies couple to the electrons.

Mele and Erwin (1993) considered the detailed Fermi surface for a system with ordered molecules. The studied an axial, nodeless order parameter with $d$-symmetry. This led to the ratio $2 \Delta / T_{c} \sim 4.6$. The temperature dependence and the quasi-particle spectrum were also nonBCS. Since the solution, however, depends on specific features of the Fermi surface, it is not clear if it survives for the disordered system.

Mele et al. (1994) studied the effects of orientational disorder. They found that this introduces some gap anisotropy. Furthermore they obtained some reduction of both $T_{c}$ and $\Delta$ in such a way that the reduced gap $2 \Delta / T_{c}$ was increased to values of the order 3.8-4.0. They therefore concluded that disorder can lead to an increased gap ratio even within a weak-coupling theory.

\section{Other properties}

Erwin and Pickett (1991) calculated the Fermi velocity $\nu_{F}$ for a system with ordered $\mathrm{C}_{60}$ molecules, using the local density approximation. From $\nu_{F}=1.810^{7} \mathrm{~cm}$ $\mathrm{s}^{-1}$ they deduced the Drude plasma energy $\hbar \Omega=1.2 \mathrm{eV}$ and the clean limit London penetration depth $\lambda=1600$ $\AA$. Considering that $\mathrm{A}_{3} \mathrm{C}_{60}$ is in the dirty limit, they corrected this result by a factor $(1+\xi / l)^{1 / 2}$, where $\xi$ is the coherence length and $l$ is the mean free path. Using $\xi=26 \AA$ and $l=10 \AA$, they obtained $\lambda \sim 3000 \AA$, in reasonable agreement with experiment (the scattering between different experiments is, however, large).

\section{CONCLUDING REMARKS}

It is widely believed that the $\mathrm{A}_{3} \mathrm{C}_{60}(\mathrm{~A}=\mathrm{K}, \mathrm{Rb})$ are $s$-wave BCS superconductors, driven by the coupling to the intramolecular $\mathrm{H}_{g}$ phonons. The work reviewed here is consistent with and supports such a picture, although there is no conclusive evidence that this picture is correct or that an electronic mechanism is excluded. Accurate and detailed tunneling data would be very interesting in this context.

It appears to be understood why $\mathrm{A}_{3} \mathrm{C}_{60}$ are metals and not Mott-Hubbard insulators, in spite of the large Coulomb interaction. Experimental and theoretical estimates of the electron-phonon coupling $\lambda$ give values of about $0.5-1$. The spread in these estimates indicate that the coupling strength is still not very accurately known. For the other important parameter determining $T_{c}$, the Coulomb pseudopotential $\mu^{*}$, there are arguments that $\mu^{*}$ is not strongly renormalized by retardation effects due to scattering of the electrons into sub bands other than the $t_{1 u}$ band. This, however, leaves interesting questions about the screening and retardation effects on $\mu^{*}$ within the $t_{1 u}$ band. Theoretical calculations of $T_{c}$ based on estimates of $\lambda$ and $\mu^{*}$ are consistent with experiment and support an electron-phonon picture.

The lattice parameter dependence of $T_{c}$ seems to be understood for $\mathrm{A}_{3} \mathrm{C}_{60}$ in the $\mathrm{Fm} \overline{3} \mathrm{~m}$ structure but not for $\mathrm{Na}_{2} \mathrm{AC}_{60}$ in the $\mathrm{Pa} \overline{3}$ structure. The doping dependence is also not well understood. The BCS like superconducting gap seen in most experiment, follows from the picture of the superconductivity being driven by the $\mathrm{H}_{g}$ intramolecular phonons, while the isotope effect still gives rise to some interesting experimental and theoretical questions.

We have emphasized that because several energy scales are comparable, the theoretical treatment of the fullerides is particularly demanding but also unusually interesting. Thus one may expect Migdal's theorem to be violated and many-body effects due to the Coulomb interaction to be important. The electron-phonon interaction should also be important for many properties. On the other hand, the molecular solid character leads to certain simplifications, for instance the electronic properties are expected to be dominated by the three-fold degenerate $t_{1 u}$ orbital. Due to the unusual parameter range, many interesting general issues are raised in a particular clear-cut way for the doped fullerides, and their solutions should have implications in many other fields.

\section{ACKNOWLEDGMENTS}


We would like to thank I.I. Mazin for many helpful suggestions.

\section{REFERENCES}

Adams, G.B., J.B. Page, O.F. Sankey, K. Sinha, J. Menendez, and D.R. Hoffman, 1991, Phys. Rev. B 44, 4052.

Aldersey-Williams, H., 1995, The most beautiful molecule, Aurum Press, London.

Allemand, P.-M., K.C. Khemani, A. Koch, F. Wudl, K. Holczer, S. Donovan, G. Grüner, and J.D. Thompson, 1991, Science 253, 301.

Allen, P.B., 1972, Phys. Rev. B 6, 2577. Observe a misprint, which was corrected by Allen (1974).

Allen, P.B., 1974, Solid State Commun. 14, 937.

Allen, P.B., and B. Mitrovic, 1982, in Solid State Physics, edited by H. Ehrenreich, D. Turnball, and F. Seitz (Academic, New York), Vol. 37, p. 1.

Anderson, P.W., 1991, "Theories of Fullerene $T_{c}$ 's which will not work", preprint.

Antropov, V.P., 1992, unpublished.

Antropov, V.P., O. Gunnarsson, and O. Jepsen, 1992, Phys. Rev. B 46, 13647.

Antropov, V.P., I.I. Mazin, O.K. Andersen, A.I. Liechtenstein, and O. Jepsen, 1993a, Phys. Rev. B 47, 12373.

Antropov, V.P., O. Gunnarsson, and A.I. Liechtenstein, 1993b, Phys. Rev. B 48, 7651.

Aryasetiawan, F., O. Gunnarsson, E. Koch, and R.M. Martin, 1996, (to be published).

Asai, Y., and Y. Kawaguchi, 1992, Phys. Rev. B 46, 1265.

Aschroft, N.W., and M. Cyrot, 1993, Europhys. Lett. 23, 605.

Auban-Senzier, P., G. Quirion, D. Jerome, P. Bernier, S. Della-Negra, C. Fabre, and A. Rassat, 1993, Synthetic Metals 56, 3027.

Auerbach, A., and G.N. Murthy, 1992, Europhys. Lett. 19, 103.

Auerbach, A., N. Manini, and E. Tosatti, 1994, Phys. Rev. B 49, 12998.

Baenitz, M., M. Heinze, K. Lüders, H. Werner, R. Schlögl, M. Weiden, G. Sparn, and F. Steglich, 1995, Solid State Commun. 96, 539.

Baggot, J., 1994, Perfect Symmetry, Oxford Press, Oxford. Baskaran, G. and E. Tosatti, 1991, Current Science 61, 33.

Belosludov, V.R., and V.P. Shpakov, 1992, Mod. Phys. Lett. B 6, 1209.

Benning, P.J., J.L. Martins, J.H. Weaver, L.P.F. Chibante, and R.E. Smalley, 1991 Science 2521417.

Benning, P.J., F. Stepniak, D.M. Poirier, J.L. Martins, J.H. Weaver, L.P.F. Chibante, and R.E. Smalley, 1993, Phys. Rev. B 47, 13843 (1993).

Berdenis, N., and G. Murthy, 1995, Phys. Rev. B 52, 3083. Berry, M.V., 1984, Proc. R. Soc. London A 392, 45.

Bethune, D.S., G. Meijer, W.C. Tang, H.J. Rosen, W.G. Golden, H. Seki, C.A. Brown, and M.S. de Vries, 1991,
Chem. Phys. Lett. 179, 181.

Bethune, D.S., 1992, as quoted by Deaven and Rokhsar (1993).

Billups, W.E., and M.A. Ciufolini, 1993, Editors Buckminsterfullerenes, VCH Publishers, New York.

Boebinger, G.S., T.T.M. Palstra, A. Passner, M.J. Rosseinsky, D.W. Murphy, and I.I. Mazin, 1992, Phys. Rev. B 46, 5876 .

Bogoliubov, N.N., V.V. Tolmachev, and D.V. Shirkov, 1958, A new method in Superconductivity, Consultants Bureau, New York.

Bohnen, K.-P., R. Heid, K.-M. Ho, and C.T. Chan, 1995, Phys. Rev. B 51, 5805.

Brühwiler, P.A., A.J. Maxwell, A. Nilsson, N. Mårtensson, and O. Gunnarsson, 1992, Phys. Rev. B 48, 18296.

Buntar, V., and H.W. Weber, 1996, Superconductor Science and Technology (in press).

Burk, B., V.H. Crespi, M.S. Fuhrer, A. Zettl, and M.L. Cohen, 1994a, Physica C 235-240, 2493.

Burk, B., V.H. Crespi, A. Zettl, and M.L. Cohen, 1994b, Phys Rev. Lett. 72, 3706.

Burkhart, G.J., and C. Meingast, 1996, (to be published).

Cappelluti, E., and L. Pietronero, 1996, Phys. Rev. B 53, 932.

Carbotte, J.P., 1990, Rev. Mod. Phys. 62, 1027.

Chakravarty, S. and S. Kivelson, 1991, Europhys. Lett. 16, 751.

Chakravarty, S., M.P. Gelfand and S. Kivelson, 1991, Science 254, 970.

Chakravarty, S., S.A. Kivelson, M.I. Salkola, and S. Tewari, 1992a, Science 256, 1306.

Chakravarty, S., S. Khlebnikov, and S. Kivelson, 1992b, Phys. Rev. Lett. 69, 212.

Chauvet, O., G. Oszlanyi, L. Forro, P.W. Stephens, M. Tegze, G. Faigel, and A. Janossy, 1994, Phys. Rev. Lett. $\mathbf{7 2}, 2721$.

Chen, C.-C, and C.M. Lieber, 1992, J. Am. Chem. Soc. 114, 3141 .

Chen, C.-C, and C.M. Lieber, 1993, Science 259, 655.

Chen, C.T., L.H. Tjeng, P. Rudolf, G. Meigs, J.E. Rowe, J. Chen, J.P. McCauley Jr, A.B. Smith III, A.R. McGhie, W.J. Romanow, and E.W. Plummer, 1991, Nature 352, 603.

Chen, G., and W.A. Goddard III, 1993, Proc. Natl. Acad. Sci. USA 90, 1350.

Chen, X.H., and G. Roth, 1995, Phys. Rev. B 52, 15534.

Chen, Y., D.M. Poirier, M.B. Jost, C. Gu, T.R. Ohno, J.L. Martins, J.H. Weaver, L.P.F. Chibante, and R.E. Smalley, 1992, Phys. Rev. B 46, 7961.

Christides, C., D.A. Neunman, K. Prassides, J.R.D. Copley, J.J. Rush, M.J. Rosseinsky, D.W. Murphy, and R.C. Haddon, 1992, Phys. Rev. B 46, 12088.

Crespi, V.H., M.L. Cohen, and D.R. Penn, 1991, Phys. Rev. B 43, 12921. 
Crespi, V.H., J.G. Hou, X.-D. Xiang, M.L. Cohen, and A. Zettl, 1992, Phys. Rev. B 46, 12064.

Deaven, D.M., and D.S. Rokhsar, 1993, Phys. Rev. B 48, 4114.

de Coulon, V., J.L. Martins, and F. Reuse, 1992, Phys. Rev. B 45, 13671.

Degiorgi, L., P. Wachter, G. Grüner, S.-M. Huang, J. Wiley, and R.B. Kaner, 1992, Phys. Rev. Lett. 69, 2987.

Degiorgi, L., G. Briceno, M.S. Fuhrer, A. Zettl, and P. Wachter, 1994, Nature 369, 541.

Degiorgi, L., 1995, Mod. Phys. Lett. B 9, 445.

De Seta, M., and F. Evangelisti, 1995, Phys. Rev. B 51, 1096.

de Vries, J., H. Steger, B. Kamke, C. Menzel, B. Weisser, W. Kamke, and I.V. Hertel, 1992, Chem. Phys. Lett. 188, 159.

Diederichs, J., J.S. Schilling, K.W. Herwig, and W.B. Yelon, 1996, J. Phys. Chem. Solids

Dolgov, O.V., and I.I. Mazin, 1992, Solid State Commun. $\mathbf{8 1 ,} 935$.

Duclos, S.J., R.C. Haddon, S. Glarum, A.F. Hebard, and K.B. Lyons, 1991, Science 254, 1625.

Ebbesen, T.W., J.S. Tsai, K. Tanigaki, J. Tabuchi, Y. Shimakawa, Y. Kubo, I. Hirosawa, and J. Mizuki, 1992a, Nature 355, 620.

Ebbesen, T.W., J.S. Tsai, K. Tanigaki, H. Hiura, Y. Shimakawa, Y. Kubo, I. Hirosawa, and J. Mizuki, 1992b, Physica C 203, 163.

Eliashberg, G.M., 1960, J. Expt. Theor. Phys. (U.S.S.R) 38, 966 [Sov. Phys. JETP 11, 696 (1960)].

Erwin, S.C., and M.R. Pederson, 1991, Phys. Rev. Lett. 67, 1610 .

Erwin, S.C., and E.J. Mele, 1994, Phys. Rev. B 505689.

Erwin, S.C. and W.E. Pickett, 1991, Science 254, 842.

Erwin, S.C. and W.E. Pickett, 1992, Phys. Rev. B 46, 14257.

Erwin, S.C., 1993, in Buckminsterfullerenes, Eds. W.E. Billups and M.A. Ciufolini (VCH Publishers, New York), p. 217.

Erwin, S.C., and C. Bruder, 1994, Physica B 199\&200, 600.

Erwin, S.C. and M.R. Pederson, 1993, Phys. Rev. B 47, 14657.

Erwin, S.C., G.V. Krishna and E.J. Mele, 1995, Phys. Rev. B 51, 7345.

Fabrizio, M, and E. Tosatti, 1996 (preprint).

Faulhaber, J.C.R., D.Y.K. Ko, and P.R. Briddon, 1993, Phys. Rev. B 48, 661.

Fischer, J.E., and D.E. Cox, 1992, Eds., Physics and Chemistry of Fullerene-Based Solids, J. Phys. Chem. Solids 53, No. 12.

Fischer, J.E., Y. Maruyama, D.E. Cox, and R.N. Shelton, 1993, Eds., Physics and Chemistry of Fullerene-Based Solids - II, J. Phys. Chem. Solids 54, No. 12.

Fischer, J.E., 1996, NATO ASI series X, Kluwer.
Fisk, Z., and G.W. Webb, 1976, Phys Rev. Lett. 36, 1084. Fleming, R.M., A.P. Ramirez, M.J. Rosseinsky, D.W. Murphy, R.C. Haddon, S.M. Zahurak, and A.V. Makhija, 1991, Nature 352, 787.

Friedberg, R., T.D. Lee and H.C. Ren, 1992, Phys. Rev. B 46, 14150.

Gelfand, M.P., and J.P. Lu, 1992a, Phys. Rev. Lett. 68, 1050.

Gelfand, M.P., and J.P. Lu, 1992b, Phys. Rev. B 46, 4367.

Gelfand, M.P., and J.P. Lu, 1993, Phys. Rev. B 47, 4149.

Gelfand, M.P., 1994, Superconductivity Review 1, 103.

Gensterblum, G., J.J. Pireaux, P.A. Thiry, R. Caudano, J.P. Vigneron, Ph. Lambin, A.A. Lucas, and W. Krätschmer, 1991, Phys Rev. Lett. 67, 2171.

Georges, A., G. Kotliar, W. Krauth, and M.J. Rozenberg, 1996, Rev. Mod. Phys. 68, 13 have given a review of the results for large dimensions.

Ginzburg, V.L., and D.A. Kirzhnits, 1982, HighTemperature superconductivity, Consultants Bureau, New York.

Goff, W.E., and P. Phillips, 1992, Phys. Rev. B 46, 603.

Goff, W.E., and P. Phillips, 1993, Phys. Rev. B 48, 3491.

Grabowski, M, and L.J. Sham, 1984, Phys. Rev. B 29, 6132.

Grimaldi, C., L. Pietronero, and S. Strässler, 1995, Phys. Rev. B 52, 10530.

Grimaldi, C., L. Pietronero, and S. Strässler, 1995b, Phys. Rev. Lett. 75, 1158.

Grimvall, G., 1981, The Electron-Phonon Interaction in Metals, (North-Holland, Amsterdam), pp. 210-223.

Gu, C., B.W. Veal, R. Liu, A.P. Paulikas, P. Kostic, H. Ding, K. Gofron, J.C. Campuzano, J.A. Schlueter, H.H. Wang, U. Geiser, and J.M. Williams, 1994, Phys. Rev. B 50, 16566.

Gunnarsson, O., S. Satpathy, O. Jepsen, and O.K. Andersen, 1991, Phys. Rev. Lett. 67, 3002.

Gunnarsson, O., and G. Zwicknagl, 1992, Phys. Rev. Lett. 69, 957.

Gunnarsson, O., D. Rainer, and G. Zwicknagl, 1992, Int. J. Mod. Phys. B 6, 3993.

Gunnarsson, O., 1993 (unpublished).

Gunnarsson, O., V. Meden, and K. Schönhammer, 1994a, Phys. Rev. B 50, 10462.

Gunnarsson, O., V. Meden, and K. Schönhammer, 1994b Progress in Fullerne Research (World Scientific, Singapore). Kuzmany, H., J. Fink, M. Mehring, and S. Roth, Eds., p. 317.

Gunnarsson, O., H. Handschuh, P.S. Bechthold, B. Kessler, G. Ganteför, and W. Eberhardt, 1995, Phys. Rev. Lett. 74, 1875.

Gunnarsson, O., 1995, Phys. Rev. B 51, 3493.

Gunnarsson, O., V. Eyert, M. Knupfer, J. Fink, J.F. Armbruster, 1996, J. Phys.: Condens. Matter 8, 2557.

Gunnarsson, O., E. Koch and R.M. Martin, 1996 (to be published). 
Gunnarsson, O., 1996 (unpublished).

Haddon, R.C., A.F. Hebard, M.J. Rosseinsky, D.W. Murphy, S.J. Duclos, K.B. Lyons, B. Miller, J.M. Rosamilia, R.M. Fleming, A.R. Kortan, S.H. Glarum, A.V. Makhija, A.J. Muller, R.H. Eick, S.M. Zahurak, R. Tycko, G. Dabbagh, and F.A. Thiel, 1991, Nature 350, 320.

Hammond, G.S., and V.J. Kuck, 1992, Editors, American Chemical Company, Washington, DC.

Hebard, A.F., M.J. Rosseinsky, R.C. Haddon, D.W. Murphy, S.H. Glarum, T.T.M. Palstra, A.P. Ramirez, and A.R. Kortan, 1991, Nature 350, 600.

Hebard, A.F., 1992, Physics Today, 45, No. 11, p. 26.

Hebard, A.F., T.T.M. Palstra, R.C. Haddon, and R.M. Fleming, 1993, Phys. Rev. B 48, 9945.

Hedin, L., and S. Lundqvis, 1969, in Solid State Physics, edited by H. Ehrenreich, D. Turnball, and F. Seitz (Academic, New York), Vol. 23, p. 1.

Hettich, R.L., R.N. Compton, and R.H. Ritchie, 1991, Phys Rev. Lett. 67, 1242.

Hirosawa, I., K. Prassides, J. Mizuki, K. Tanigaki, M. Gevaert, A. Lappas, and J.K. Crockcroft, 1994, Science, 264, 1294.

Hohenberg, P., and W. Kohn, 1964, Phys. Rev. 136, B864.

Holczer, K., O. Klein, S.-M. Huang, R.B. Kaner, K.-J. Fu, R.L. Whetten, and F. Diederich, 1991a, Science 252, 1154.

Holczer, K., O. Klein, G. Grüner, J.D. Thompson, F. Diederich, and R.L. Whetten, 1991b, Phys. Rev. Lett. 67, 271.

Holczer, K. and R.L. Whetten, 1992, Carbon 30, 1261.

Hou, J.G., V.H. Crespi, X.-D. Xiang, W.A. Vareka, G. Briceno, A. Zettl, and M.L. Cohen, 1993, Solid State Commun. 86, 643.

Hou, J.G., L. Lu, V.H. Crespi, X.-D. Xiang, A. Zettl, and M.L. Cohen, 1995, Solid State Commun. 93, 973.

Huang, M.-Z., Y.-N. Xu and W.Y. Ching, 1992, Phys. Rev. B 46, 6572 .

Ihm, J., 1994, Phys. Rev. B 49, 10726.

Ikeda, M.A., A. Ogasawara and M. Sugihara, 1992, Phys. Lett. A 170, 319.

Inada, Y., and K. Nasu, 1992, J. Phys. Soc. Japan 61, 4511.

Jahn, H.A., and E. Teller, 1937, Proc. R. Soc. London A 161, 220 .

Jess, P., S. Behler, M. Bernasconi, V. Thommen-Geiser, H.P. Lang, M. Baenitz, K. Lüders, and H.-J. Güntherodt, 1994, Physica C 235-240, 2499..

Jess, P., U. Hubler, S. Behler, V. Thommen-Geiser, H.P. Lang, and H.-J. Güntherodt, 1996, Synthetic Metals 77, 201.

Jishi, R.A., and M.S. Dresselhaus, 1992, Phys. Rev. B 45, 2597.

Jishi, R.A., R.M. Miere, and M.S. Dresselhaus, 1992, Phys. Rev. B 45, 13685.
Jones, R.O., and O. Gunnarsson, 1989, Rev. Mod. Phys. $\mathbf{6 1}, 689$.

Jones, R., C.D. Latham, M.I. Heggie, V.J.B. Torres, S. Öberg, and S.K. Estreicher, 1992, Phis. Mag. Lett. 65, 291.

Joubert, D.P., 1993, J. Phys.: Condens. Matter 5, 8047.

Kiefl, R.F., T.L. Duty, J.W. Schneider, A. MacFarlane, K. Chow, J.W. Elzey, P. Mendels, G.D. Morris, J.H. Brewer, E.J. Ansaldo, C Niedermayer, D.R. Noakes, C.E. Stronach, B. Hitti, and J.E. Fischer, 1992, Phys. Rev. Lett. 69, 2005.

Kiefl, R.F., W.A. MacFarlane, K.H. Chow, S. Dunsiger, T.L. Duty, T.M.S. Johnston, J.W. Schneider, J. Sonier, L. Brard, R.M. Strongin, J.E. Fischer, and A.B. Smith III, 1993, Phys Rev. Lett. 70, 3987.

Kniaz, K, J.E. Fischer, Q. Zhu, M.J. Rosseinsky, O. Zhou, and D.W. Murphy, 1993, Solid State Commun 88, 47.

Knupfer, M., M. Merkel, M.S. Golden, J. Fink, O. Gunnarsson, V.P. Antropov, 1993, Phys. Rev. B 47, 13944.

Knupfer, M., F. Stepniak, and J.H. Weaver, 1994, Phys. Rev. B 49, 7620.

Knupfer, M., J. Fink, J.F. Armbruster, and H.A. Romberg, 1995, Z. Phys. 98, 9.

Kohanoff, J., W. Andreoni, and M. Parrinello, 1992, Phys. Rev. B 46, 4371.

Kohn, W., and L.J. Sham, 1965, Phys. Rev. 140, A1133.

Kortan, A.R., N. Kopylov, S. Glarum, E.M. Gyorgy, A.P. Ramirez, R.M. Fleming, F.A. Thiel, and R.C. Haddon, 1992a, Nature 355, 529.

Kortan, A.R., N. Kopylov, S. Glarum, E.M. Gyorgy, A.P. Ramirez, R.M. Fleming, O. Zhou, F.A. Thiel, P.L. Trevor, and R.C. Haddon, 1992b, Nature 360, 566.

Kortan, A.R., N. Kopylov, E. Özdas, A.P. Ramirez, R.M. Fleming, and R.C. Haddon, 1994, Chem. Phys. Lett. 223, 501.

Kostur, V.N., and B. Mitrovic, 1993, Phys. Rev. B 48, 16388.

Kostur, V.N., and B. Mitrovic, 1994, Phys. Rev. B 50, 12774 .

Krätschmer, W., L.D. Lamb, K. Fostiropoulos, and D.R. Huffman, 1990, Nature 347, 354.

Krier, G., and O. Gunnarsson, 1995 (unpublished).

Krishnamurthy, H.R., D.M. Newns, P.C. Pattnaik, C.C. Tsuei, and C.C. Chi, 1994, Phys. Rev. B 49, 3520.

Kroto, H.W., J.R. Heath, S.C. O'Brien, R.F. Curl, and R.E. Smalley, 1985 Nature 318, 162.

Kroto, H.W., A. W. Allaf, and S.P. Balm, 1991, Chem. Rev. 91, 1213.

Kulic, M.L., and R. Zheyer, 1994, Phys. Rev. B 49, 4395.

Kumar, V., T.P. Martin, and E. Tosatti, 1992, Editors, Clusters and fullerenes, Int. J. Mod. Phys. B 6, No. $23 \& 24$.

Kuzmany, H., J. Fink, M. Mehring, and S. Roth, 1993, Eds., Electronic properties of fullerenes (Springer, Berlin). 
Kuzmany, H., J. Fink, M. Mehring, and S. Roth, 1994a, Eds., Progress in Fullerne Research (World Scientific, Singapore).

Kuzmany, H., J. Fink, M. Mehring, and S. Roth, 1995a, Eds., Physics and chemistry of fullerenes and derivatives (World Scientific, Singapore).

Kuzmany, H., M. Matus, B. Burger, and J. Winter, 1994b, Adv. Mater. 6, 731.

Kuzmany, H., R. Winkler, and T. Pichler, 1995b, J. Phys.: Cond. Matter 7, 6601.

Lammert, P.E., and D.S. Rokhsar, 1993, Phys. Rev. B 48, 4103.

Lammert, P.E., D.S. Rokhsar, S. Chakravarty, S. Kivelson, and M.I. Salkola, 1995, Phys. Rev. Lett. 74, 996.

Lannin, J.S., and M.G. Mitch, 1994, Phys. Rev. B 50, 6497.

Lannoo, M., G.A. Baraff, M. Schluter, and D. Tomanek, 1991, Phys. Rev. B 44, 12106.

Laouini, N., O.K. Andersen, O. Gunnarsson, 1995, Phys. Rev. B 51, 17446.

Lieber, C.M., and Z. Zhang, 1994, Solid State Physics, Edited by H. Ehrenreich and F. Spaepen, Academic Press, New York, p. 349.

Liechtenstein, A.I., O. Gunnarsson, M. Knupfer, J. Fink, and J.F. Armbruster, 1996, J. Phys.: Condens. Matter 8, 4001.

Limbach, P.A., L. Schweikhard, K.A. Cowen, M.T. McDermott, A.G. Marshall, and J.V. Coe, 1991, J. Am. Chem. Soc. 113, 6795.

Lin, C.L., T. Mihalisin, N. Bykovetz, Q. Zhu, and J.E. Fischer, 1994, Phys. Rev. B 49, 4285.

Lof, R.W., M.A. van Veenendaal, B. Koopmans, H.T. Jonkman, and G.A. Sawatzky, 1992, Phys. Rev. Lett. 68, 3924 .

Loktev, V.M., 1992, Fiz. Nizk. Temp. 18, 217 [Sov. J. Low Temp. Phys. 18, 149].

Lu, J.P., 1994, Phys. Rev. B 49, 5687.

Manini, N., E. Tosatti, and A. Auerbach, 1994, Phys. Rev. B 49, 13008.

Maniwa, Y., T. Saito, K. Kume, K. Kikuchi, I. Ikemoto, S. Suzuki, Y. Achiba, I. Hirozawa, M. Kosaka, and K. Tanigaki, 1995, Phys. Rev. B 52, R7054.

Martin, R.L., and J.P. Ritchie, 1993, Phys. Rev. B 48, 4845 .

Martins, J.L., and N. Troullier, 1992, Phys. Rev. B 46, 1766.

Mazin, I.I., S.N. Rashkeev, V.P. Antropov, O. Jepsen, A.I. Liechtenstein, and O.K. Andersen, 1992, Phys. Rev. B 45, 5114 .

Mazin, I.I., A.I. Liechtenstein, O. Gunnarsson, O.K. Andersen, V.P. Antropov, and S.E. Burkov, 1993a, Phys. Rev. Lett. 70, 4142.

Mazin, I.I., O.V. Dolgov, A. Golubov, and S.V. Shulga, 1993b, Phys. Rev. B 47, 538.

McMillan, W.L., 1968, Phys. Rev. 167, 331.
Mele, E.J., and S.C. Erwin, 1993, Phys. Rev. B 47, 2948. Mele, E.J., and S.C. Erwin, 1994, Phys. Rev. B 50, 2150. Mele, E.J., S.C. Erwin, and M.S. Deshpande, 1994, Synthetic Metals 65, 255.

Mitch, M.G., S.J. Chase, and J.S. Lannin, 1992a, Phys. Rev. Lett. 68, 883.

Mitch, M.G., S.J. Chase, and J.S. Lannin, 1992b, Phys. Rev. B 46, 3696.

Mitch, M.G., and J.S. Lannin, 1993, Phys. Rev. B 48, 16192.

Mitrovic, B., H.G. Zarate, and J.P. Carbotte, 1984, Phys. Rev. B 29, 184.

Migdal, A.B., 1958, J. Expt. Theor. Phys. (U.S.S.R.) 34, 1438 [Sov. Phys. JETP 7, 996 (1958)].

Mizuki, J., M. Takai, H. Takahashi, N. Mori, K. Tanigaki, I. Hirosawa, and K. Prassides, 1994, Phys. Rev. B 50, 3466.

Morel, P., and P.W. Anderson, 1992, Phys. Rev. 125, 1263.

Murphy, D.W., M.J. Rosseinsky, R.M. Fleming, R. Tycko, A.P. Ramirez, R.C. Haddon, T. Siegrist, G. Dabbagh, J.C. Tully, and R.E. Walstedt, 1992, J. Phys. Chem. Solids 53, 1321.

Murthy, G.N., and A. Auerbach, 1992, Phys. Rev. B 46, 331.

Nambu, Y., 1960, Phys. Rev. 117, 648.

Negri, F., G. Orlandi, and F. Zerbetto, 1988, Chem. Phys. Lett. 144, 31.

Novikov, D.L., V.A. Gubanov and A.J. Freeman, 1992, Physica C 191, 399.

Onida, G., and G. Benedek, 1992, Europhys. Lett. 18, 403.

Özdas, E., A.R. Kortan, N. Kopylov, A.P. Ramirez, T. Siegrist, K.M. Rabe, H.E. Bair, S. Schuppler, and P.H. Citrin, 1995, Nature, 375, 126.

Palstra, T.T.M., A.F. Hebard, R.C. Haddon, and P.B. Littlewood, 1994, Phys. Rev. B 50, 3462.

Palstra, T.T.M., O. Zhou, Y. Iwasa, P.E. Sulewski, R.M. Fleming, and B.R. Zegarski, 1995, Solid State Commun. 93, 327.

Parks, R.D., 1969, Superconductivity (Dekker, New York).

Pederson, M.R., and A.A. Quong, 1992, Phys. Rev. B 46, 13584.

Pichler, T., M. Matus, J. Kürti, and H. Kuzmany, 1992, Phys. Rev. B 45, 13841.

Pickett, W.E., 1994, Solid State Physics, Edited by H. Ehrenreich and F. Spaepen, Academic Press, New York, p. 226.

Pickett, W.E., D.A. Papaconstantopoulos, M.R. Pederson, and S.C. Erwin, 1994, J. Superconductivity, 7, 651. The values of $\lambda$ quoted here differ from the ones given by Pickett et al., due to a different convention used by them.

Pietronero, L., and S. Strässler, 1992, Europhys. Lett. 18, 627. 
Pietronero, L., S. Strässler, and C. Grimaldi, 1995, Phys. Rev. B 52, 10516.

Pintschovius, L., 1996, Rep. Prog. Phys. 59, 473.

Prassides, K., J. Tomkinson, C. Christides, M.J. Rosseinsky, D.W. Murphy, and R.C. Haddon, 1991, Nature 354, 462.

Prassides, K., C. Christides, M.J. Rosseinsky, J. Tomkinson, D.W. Murphy, and R.C. Haddon, 1992, Europhys. Lett. 19, 629.

Prassides, K., C. Christides, I.M. Thomas, J. Mizuki, K. Tanigaki, I. Hirosawa, and T.W. Ebbesen, 1994, Science 263, 950.

Prassides, K., 1994, Editor, Physics and Chemistry of the fullerenes, NATO ASI series, Kluwer Academic Publishers, Dordrect.

Quong, A.A., M.R. Pederson, and J.L. Feldman, 1993, Solid State Commun. 87, 535.

Rainer, D., 1986, Prog. Low Temp. Phys. 10, 371.

Ramirez, A.P., A.R. Kortan, M.J. Rosseinsky, S.J. Duclos, A.M. Mujsce, R.C. Haddon, D.W. Murphy, A.V. Makhija, S.M. Zahurak, and K.B. Lyons, 1992a, Phys Rev. Lett. 68, 1058.

Ramirez, A.P., M.J. Rosseinsky, D.W. Murphy, and R.C. Haddon, 1992b, Phys Rev. Lett. 69, 1687.

Ramirez, A.P., 1994, Superconductivity Review 1, 1.

Ramirez, A.P., M.J. Rosseinsky, and D.W. Murphy, 1994, as quoted in Ramirez (1994).

Renker, B., F. Gompf, H. Schober, P. Adelmann, H.J. Bornemann, and R. Heid, 1993, Z. Physik. B 92, 451.

Reznik, D., W.A. Kamitakahara, D.A. Neumann, J.R.D. Copley, J.E. Fischer, R.M. Strongin, M.A. Cichy, and A.B. Smith III, 1994, Phys. Rev. B 49, 1005.

Rietschel, H., and L.J. Sham, 1983, Phys. Rev. B 28, 5100.

Rosseinsky, M.J., A.P. Ramirez, S.H. Glarum, D.W. Murphy, R.C., Haddon, A.F. Hebard, T.T.M. Palstra, A.R. Kortan, S.M. Zahurak, and A.V. Makhija, 1991, Phys. Rev. Lett. 66, 2830.

Rosseinsky, M.J., D.W. Murphy, R.M. Fleming, and O. Zhou, 1993, Nature 364, 425.

Rotter, L.D., Z. Schlesinger, J.P. McCauley Jr, N. Coustel, J.E. Fischer, and A.B. Smith III, 1992, Nature 355, 532.

Saito, S., and A. Oshiyama, 1991a, Phys. Rev. Lett. 66, 2637.

Saito, S., and A. Oshiyama, 1991b, Phys. Rev. 44, 11536.

Saito, S., and A. Oshiyama, 1993, Phys. Rev. Lett. 71, 121.

Sasaki, S., A. Matsuda, and C.W. Chu, 1994, J. Phys. Soc. Japan 63, 1670.

Satpathy, S., V.P. Antropov, O.K. Andersen, O. Jepsen, O. Gunnarsson, and A.I. Liechtenstein, 1992, Phys. Rev. B 46, 1773.

Sawatzky, G.A., 1995, in Physics and chemistry of fullerenes and derivatives Kuzmany, H., J. Fink, M.
Mehring, and S. Roth, Eds., (World Scientific, Singapore) p. 373 .

Scherrer, H., and G. Stollhoff, 1993, Phys. Rev. B 47, 16570 .

Schirber, J.E., D.L. Overmyer, W.R. Bayless, M.J. Rosseinsky. D.W. Murphy, Q. Zhu, O. Zhou, K. Kniaz, and J.E. Fischer, 1993, J. Phys. Chem. Solids 54, 1427.

Schluter, M., M. Lannoo, M. Needels, G.A. Baraff, and D. Tomanek, 1992a, Phys. Rev. Lett. 68, 526.

Schluter, M., M. Lannoo, M. Needels, G.A. Baraff, and D. Tomanek, 1992b, J. Phys. Chem. Solids 53, 1473.

Schluter, M.A., M. Lannoo, M.F. Needels, G.A. Baraff and D. Tomanek, 1992c, Phys Rev. Lett. 69, 213.

Schober, H., B. Renker, F. Gompf, and P. Adelmann, 1994, Physica 235-240C, 2487.

Schrieffer, J.R., Theory of superconductivity, 1964, Benjamin, New York.

Sparn, G., J.D. Thompson, S.-M. Huang, R.B. Kaner, F. Diederich, R.L. Wetten, G. Grüner, and K. Holczer, 1991, Science 252, 1829.

Sparn, G., J.D. Thompson, R.L. Whetten, S.-M. Huang, R.B. Kaner, F. Diederich, G. Grüner, and K. Holczer, 1992, Phys. Rev. Lett. 68, 1228.

Stenger, V.A., C.H. Pennington, D.R. Buffinger, and R.P. Ziebarth, 1995, Phys. Rev. Lett. 74, 1649.

Stephens, P.W., L. Mihaly, P.L. Lee, R.L. Whetten, S.-M. Huang, R. Kaner, F. Deiderich, and K. Holczer, 1991, Nature 351, 632.

Stephens, P.W., G. Bortel, G. Falgel, M. Tegze, A. Janossy, S. Pekker, G. Oszlanyl, and L. Forro, 1994, Nature 370, 636.

Stollhoff, G., 1991, Phys. Rev. B 44, 10998.

Takada, Y., 1993, J. Phys. Chem. Solids 54, 1779.

Tanigaki, K., T.W. Ebbesen, S. Saito, J. Mizuki, J.S. Tsai, Y. Kubo, and S. Kuroshima, 1991, Nature 352, 222.

Tanigaki, K., I. Hirosawa, T.W. Ebbesen, J. Mizuki, Y. Shimakawa, Y. Kubo, J.S. Tsai, and S. Kuroshima, 1992, Nature 356, 419.

Tanigaki, K., I. Hirosawa, T.W. Ebbesen, J.-I. Mizuki, and J.-S. Tsai, 1993, J. Phys. Chem. Solids 54, 1645.

Tanigaki, K., M. Kosaka, T. Manako, Y. Kubo, I. Hirosawa, K. Uchida, and K. Prassides, 1995, Chem. Phys. Lett. 240, 627.

Tanigaki, K., and K. Prassides, 1995, J. Mater. Chem. 5, 1515.

Teslic, S., T. Egami, and J.E. Fischer, 1995, Phys. Rev. B $\mathbf{5 1 ,} 5973$.

Tinkham, M., 1975, Introduction to superconductivity, (McGraw-Hill, New-York).

Tokuyasu, T., M. Kamal and G. Murthy, 1993, Phys Rev. Lett. 71, 4202.

Troullier, N. and J.L. Martins, 1992, Phys. Rev. B 46, 1754.

Tycko, R., G. Dabbagh, M.J. Rosseinsky, D.W. Murphy, A.P. Ramirez, and R.M. Fleming, 1992, Phys Rev. Lett. 
68, 1912.

Tycko, R., G. Dabbagh, D.W. Murphy, Q. Zhu and J.E. Fischer, 1993, Phys. Rev. B 48, 9097.

Uemura, Y.J., A. Keren, L.P. Le, G.M. Luke, B.J. Sternlieb, W.D. Wu, J.H. Brewer, R.L. Whetten, S.M. Huang, S. Lin, R.B. Kaner, F. Diederich, S. Donovan, G. Grüner, and K. Holczer, 1991, Nature 352, 605.

Uemura, Y.J., L.P. Lee, and G.M. Luke, 1992, Synthetic Metals 57, 2845.

Vareka, W.A., and A. Zettl, 1994, Phys. Rev. Lett. 72, 4121.

Varma, C.M., J. Zaanen, K. Raghavachari, 1991, Science 254, 989 .

Walstedt, R.E., D.W. Murphy and M. Rosseinsky, 1993 , Nature 362, 611.

Wang, D., 1995, Solid State Commun. 94, 767.

Wang, Y., D. Tomanek, and G.F. Bertsch, 1991, Phys. Rev. B 44, 6562 .

Ward, J.C., 1950, Phys. Rev. 78, 182.

Weltner Jr., W., and R.J. van Zee, 1989, Chem. Rev. 89, 1713.

Wertheim, G.K., D.N.E. Buchanan, and J.E. Rowe, 1992, Science 258, 1638.

Wertheimer, N.R., E. Helfand, and P.C. Hohenberg, 1966, Phys. Rev. 147, 295.

White, S.R., S. Chakravarty, M.P. Gelfand, and S. A. Kivelson, 1992, Phys. Rev. B 45, 5062.

Wilson, M.A., L.S. Pang, G.D. Willett, K.J Fisher and I.G. Dance, 1992, Carbon 30, 675.

Winter, J. and H. Kuzmany, 1996, Phys. Rev. B 53, 655.

Wong, W.H., M.E. Hanson, W.G. Clark, G. Grüner, J.D. Thompson, R.L. Whetten, S.-M. Huang, R.B. Kaner, F. Diederich, P. Petit, J.-J. Andre and K. Holczer, 1992, Europhys. Lett. 18, 79.

Xiang, X.-D., J.G. Hou, G. Briceno, W.A. Vareka, R. Mostovoy, A. Zettl, V.H. Crespi, and M.L. Cohen, 1992, Science 256, 1190.

Xiang, X.-D., J.G. Hou, V.H. Crespi, A. Zettl, and M.L. Cohen, 1993, Nature 361, 54.

Yabana, K., and G. Bertsch, 1992, Phys. Rev. B 46, 14263.

Yildirim, T., J.E. Fischer, R. Dinnebier, P.W. Stephens, and C.L. Lin, 1994,

Yildirim, T., J.E. Fischer, R. Dinnebier, P.W. Stephens, and C.L. Lin, 1995, Solid State Commun. 93, 269.

Yildirim, T., L. Barbedette, K. Kniaz, J.E. Fischer, C.L. Lin, N. Bykovetz, P.W. Stephens, P.E. Sulewski, and S.C. Erwin, 1995, in Science and technology of fullerene materials, Eds. P. Bernier, T.W. Ebbesen, D.S. Bethune, R.M. Metzger, L.Y. Chiang, and J.W. Mintmire (Mat. Res. Soc. Proc. 359, Pittsburg, 1995) p. 273.

Yildirim, T., L. Barbedette, J.E. Fischer, C.L. Lin, J. Robert, P. Petit, and T.T. M. Palstra, 1996a, Phys. Rev. Lett. 77, 167.

Yildirim, T., L. Barbedette, J.E. Fischer, G. Bendele, P.W. Stephens, C.L. Lin, C. Goze, F. Rachdi, J. Robert, P.
Petit, and T.T.M. Palstra, 1996b, Phys. Rev. B (submitted).

Zakhidov, A.A., K. Imaeda, D.M. Petty, K. Yakushi, H. Inokuchi, K. Kikuchi, I. Ikemoto, S. Suzuki, and Y. Achiba, 1992, Phys. Lett. A 164, 355.

Zhang, F.C., M. Ogata, and T.M. Rice, 1991a, Phys. Rev. Lett. 67, 3452 .

Zhang, W., H. Zheng, and K.H. Bennemann, 1992, Solid State Commun. 82, 679.

Zhang, Z., C.-C. Chen, S.P. Kelty, H. Dai, and C.M. Lieber, 1991b, Nature 353, 333.

Zheng, H. and K.H. Bennemann, 1992, Phys. Rev. B 46, 11993.

Zheyer, R., and G. Zwicknagl, 1990, Z. Phys. B 78, 175.

Zhou, O., G.B.M. Vaughan, Q. Zhu, J.E. Fischer, P.A. Heiney, N. Coustel, J.P. McCauley, Jr., and A.B. Smith III, 1992, Science 255, 833.

Zhou, O., R.M. Fleming, D.W. Murphy, M.J. Rosseinsky, A.P. Ramirez, R.B. van Dover, and R.C. Haddon, 1993, Nature 362, 433.

Zhou, O., T.T.M. Palstra, Y. Iwasa, R.M. Fleming, A.F. Hebard, P.E. Sulewski, D.W. Murphy, and B.R. Zegarski, 1995 Phys. Rev. B 52, 483.

Zhu, Q., Phys. Rev. B 52, R723 (1995).

Zimmer, G., M. Helmle, M. Mehring, F. Rachdi, J. Reichenbach, L. Firlej, and P. Bernier, 1993, Europhys. Lett. 24, 59. 